\title{
Completing the scalar and fermionic universal one-loop effective action
}

\section{Michael Krämer, Benjamin Summ and Alexander Voigt}

Institute for Theoretical Particle Physics and Cosmology, RWTH Aachen University, 52074 Aachen, Germany

E-mail: mkraemer@physik.rwth-aachen.de, benjamin.summ@rwth-aachen.de, alexander.voigt@physik.rwth-aachen.de

ABSTRACT: We extend the known Universal One-Loop Effective Action (UOLEA) by all operators which involve scalars and fermions, not including contributions arising from open covariant derivatives. Our generic analytic expressions for the one-loop Wilson coefficients of effective operators up to dimension six allow for an application of the UOLEA to a broader class of UV-complete models. We apply our generic results to various effective theories of supersymmetric models, where different supersymmetric particles are integrated out at a high mass scale.

KeYwords: Effective Field Theories, Beyond Standard Model

ArXiv EPrint: 1908.04798 


\section{Contents}

1 Introduction 1

2 Calculation of the scalar and fermionic UOLEA 3

2.1 Functional matching in a scalar theory 3

2.2 Fermionic contributions to the UOLEA 5

3 Discussion of the result $\quad 12$

$\begin{array}{lll}3.1 & \text { Published operators and coefficients } & 12\end{array}$

$\begin{array}{lll}3.2 & \text { Infrared and ultra-violet divergences } & 14\end{array}$

$\begin{array}{ll}3.3 & \text { Application to models with massive vector fields } \\ \end{array}$

$\begin{array}{lll}3.4 & \text { Extraction of } \beta \text {-functions } & 15\end{array}$

4 Applications $\quad 16$

$\begin{array}{ll}\text { 4.1 Integrating out the top quark from the Standard Model } & 16\end{array}$

$\begin{array}{lll}\text { 4.2 } & \text { MSSM threshold correction to the quartic Higgs coupling } & 17\end{array}$

4.3 Integrating out stops and the gluino from the MSSM 23

4.4 Integrating out the gluino from the MSSM with light stops 26

5 Conclusions $\quad 32$

$\begin{array}{ll}\text { A Fermionic shifts } & 33\end{array}$

B Loop functions $\quad 35$

C Useful relations for spinors and $\mathrm{SU}(N)$ groups $\quad 36$

$\begin{array}{ll}\text { D Dimensional regularization and dimensional reduction } & 37\end{array}$

\section{Introduction}

With the discovery of the Higgs boson at the Large Hadron Collider (LHC) [1, 2], the Standard Model of Particle Physics (SM) is formally complete. While existing deviations between some SM predictions and experiment, such as for the anomalous magnetic moment of the muon (see for example [3, 4]), are not conclusive, the SM is not a complete description of nature as it neither accounts for astrophysical phenomena such as dark matter, nor does it incorporate gravity.

Searches for physics beyond the SM have not been successful thus far. Exclusion limits for new particles introduced by SM extensions often exceed the TeV scale. These results suggest that new physics either interacts weakly with the SM, or that the masses 
of new particles are significantly above the electroweak scale. A well-known example is the Minimal Supersymmetric Standard Model (MSSM) [5], which requires at least TeV-scale stops in order to correctly predict the mass of the SM-like Higgs boson of about $125 \mathrm{GeV}$, see for example [6,7]. The construction and phenomenological analysis of new physics models with heavy particles is therefore a suitable path to develop viable theories beyond the SM that are consistent with experimental results.

The observables predicted in models with large mass hierarchies, however, usually suffer from large logarithmic quantum corrections, which should be resummed in order to obtain precise predictions. Effective Field Theories (EFTs) are a well-suited tool to resum these large logarithmic corrections. Conventional matching procedures using Feynman diagrams, however, are often cumbersome, in particular if the new physics model contains many new heavy particles and/or complicated interactions. The Universal One-Loop Effective Action (UOLEA) [8-10], which has been developed using functional methods [11-18], is a very promising tool to overcome these difficulties. It represents a generic one-loop expression for the Wilson coefficients of an effective Lagrangian for a given ultra-violet (UV) model with a large mass hierarchy. Compared to the conventional matching using Feynman diagrams, the calculation of the Wilson coefficients with the UOLEA is straightforward, as it is expressed directly in terms of derivatives of the UV Lagrangian w.r.t. the fields and simple rational functions. In particular, no loop integration is necessary and spurious infrared (IR) divergences are absent by construction. To date, however, the UOLEA is not completely known: only contributions from scalar particles [8,9] as well as conversion terms between dimensional regularization and dimensional reduction [10] have been calculated at the generic one-loop level up to dimension 6. Whereas some contributions from fermion loops can be calculated using these results by squaring the fermionic trace, this treatment is incomplete when the couplings depend on gamma matrices. Furthermore, contributions from loops containing both scalars and fermions as well as terms with open covariant derivatives are unknown.

In this publication we present all one-loop operators of the UOLEA up to dimension 6 that involve both scalars and fermions in a generic form, excluding contributions from open covariant derivatives. Thus, our results go beyond the scope of $[8,9]$ and allow for an application of the UOLEA to a broader set of new physics models. We publish our generic expressions in a Mathematica file UOLEA.m of the arXiv submission. Due to their generic structure, the expressions are well suited to be implemented into generic spectrum generators such as SARAH [19-22] or FlexibleSUSY [23, 24] or EFT codes in the spirit of CoDEx $[25,26]$.

This paper is structured as follows: in section 2 we present the calculation of the UOLEA involving both scalars and fermions. We discuss the results in section 3 and apply our generic expressions to various EFTs of the SM and the MSSM in section 4. Our conclusions are presented in section 5, and the appendices collect further formulae and calculational details. 


\section{Calculation of the scalar and fermionic UOLEA}

\subsection{Functional matching in a scalar theory}

In this section we briefly review the most important steps in the functional matching approach at one-loop level in a scalar theory and fix the notation for the subsequent sections. Most of what is being discussed here is well-documented in the literature and more details can be found in $[14,15,17,18]$. We consider a generic UV theory that contains heavy real scalar fields, collectively denoted by $\Phi$, with masses of the order $M$ and light real scalar fields, denoted by $\phi$, with masses of the order $m$. We assume that $m / M \ll 1$ such that an EFT expansion in the mass ratio $m / M$ is valid. To perform the functional matching the background field method is used to calculate the generator of 1-light-particle-irreducible (1LPI) Green's functions in the UV-theory, $\Gamma_{\mathrm{L}, \mathrm{UV}}\left[\phi_{\mathrm{cl}}\right]$, and the generator of 1-particleirreducible (1PI) Green's functions in the EFT, $\Gamma_{\mathrm{EFT}}\left[\phi_{\mathrm{cl}}\right]$, where $\phi_{\mathrm{cl}}$ are light background fields which obey the classical equation of motion. For the determination of these generating functionals beyond tree-level a regularization scheme must be specified, which is chosen to be dimensional regularization. ${ }^{1}$ This introduces a dependence on the unphysical renormalization scale $\mu$ in both generating functionals, and the matching condition becomes

$$
\Gamma_{\mathrm{L}, \mathrm{UV}}\left[\phi_{\mathrm{cl}}\right]=\Gamma_{\mathrm{EFT}}\left[\phi_{\mathrm{cl}}\right]
$$

which is imposed at the matching scale $\mu$, order by order in perturbation theory. In principle the matching scale can be chosen arbitrarily, however, in order to avoid large logarithms the choice $\mu=M$ is preferred. To calculate $\Gamma_{\mathrm{L}, \mathrm{UV}}\left[\phi_{\mathrm{cl}}\right]$ one starts from the generating functional of Green's functions

$$
Z_{\mathrm{UV}}\left[J_{\Phi}, J_{\phi}\right]=\int \mathcal{D} \Phi \mathcal{D} \phi \exp \left\{i \int \mathrm{d}^{d} x\left[\mathcal{L}_{\mathrm{UV}}[\Phi, \phi]+J_{\Phi}(x) \Phi(x)+J_{\phi}(x) \phi(x)\right]\right\}
$$

with sources $J_{\Phi}$ and $J_{\phi}$ and splits both the heavy and the light fields into background parts $\Phi_{\mathrm{cl}}$ and $\phi_{\mathrm{cl}}$, respectively, and fluctuations $\delta \Phi$ and $\delta \phi$, respectively, as

$$
\begin{aligned}
\Phi & =\Phi_{\mathrm{cl}}+\delta \Phi, \\
\phi & =\phi_{\mathrm{cl}}+\delta \phi .
\end{aligned}
$$

The background fields are defined to satisfy the classical equations of motion,

$$
\frac{\delta \mathcal{L}_{\mathrm{UV}}}{\delta \Phi}\left[\Phi_{\mathrm{cl}}, \phi_{\mathrm{cl}}\right]+J_{\Phi}=0, \quad \frac{\delta \mathcal{L}_{\mathrm{UV}}}{\delta \phi}\left[\Phi_{\mathrm{cl}}, \phi_{\mathrm{cl}}\right]+J_{\phi}=0 .
$$

The generating functional of the 1LPI Green's functions of the UV model, $\Gamma_{\mathrm{L}, \mathrm{UV}}\left[\phi_{\mathrm{cl}}\right]$, is then given by

$$
\Gamma_{\mathrm{L}, \mathrm{UV}}\left[\phi_{\mathrm{cl}}\right]=-i \log Z_{\mathrm{UV}}\left[J_{\Phi}=0, J_{\phi}\right]-\int \mathrm{d}^{d} x J_{\phi}(x) \phi_{\mathrm{cl}}(x),
$$

\footnotetext{
${ }^{1}$ In principle the results obtained in this paper can also be applied to a setting where dimensional reduction is used as a regularization scheme, see [10].
} 
where $J_{\Phi}=0$ since we are only interested in Green's functions with light external particles. Expanding the Lagrangian together with the source terms around the background fields yields

$$
\begin{aligned}
\mathcal{L}_{\mathrm{UV}}[\Phi, \phi]+J_{\Phi} \Phi+J_{\phi} \phi= & \mathcal{L}_{\mathrm{UV}}\left[\Phi_{\mathrm{cl}}, \phi_{\mathrm{cl}}\right]+J_{\Phi} \Phi_{\mathrm{cl}}+J_{\phi} \phi_{\mathrm{cl}} \\
& -\frac{1}{2}\left(\begin{array}{ll}
\delta \Phi^{T} & \delta \phi^{T}
\end{array}\right) \mathcal{Q}\left(\begin{array}{c}
\delta \Phi \\
\delta \phi
\end{array}\right)+\cdots
\end{aligned}
$$

where the matrix

$$
\mathcal{Q} \equiv-\left(\begin{array}{cc}
\frac{\delta^{2} \mathcal{L}_{\mathrm{UV}}}{\delta \Phi \delta \Phi}\left[\Phi_{\mathrm{cl}}, \phi_{\mathrm{cl}}\right] & \frac{\delta^{2} \mathcal{L}_{\mathrm{UV}}}{\delta \Phi \delta \phi}\left[\Phi_{\mathrm{cl}}, \phi_{\mathrm{cl}}\right] \\
\frac{\delta^{2} \mathcal{L}_{\mathrm{UV}}}{\delta \phi \delta \Phi}\left[\Phi_{\mathrm{cl}}, \phi_{\mathrm{cl}}\right] & \frac{\delta^{2} \mathcal{L}_{\mathrm{UV}}}{\delta \phi \delta \phi}\left[\Phi_{\mathrm{cl}}, \phi_{\mathrm{cl}}\right]
\end{array}\right)
$$

is referred to as the fluctuation operator and the dots indicate higher order terms in the expansion. Through the equations of motion with $J_{\Phi}=0$ the heavy background fields can be expressed in terms of the light ones such that $\Phi_{\mathrm{cl}}=\Phi_{\mathrm{cl}}\left[\phi_{\mathrm{cl}}\right]$. In general, $\Phi_{\mathrm{cl}}\left[\phi_{\mathrm{cl}}\right]$ is a non-local object and has to be expanded using a local operator expansion. The one-loop part of $\Gamma_{\mathrm{L}, \mathrm{UV}}\left[\phi_{\mathrm{cl}}\right]$ is then found to be

$$
\Gamma_{\mathrm{L}, \mathrm{UV}}^{1 \ell}\left[\phi_{\mathrm{cl}}\right]=\frac{i}{2} \log \operatorname{det} \mathcal{Q} .
$$

The above can be re-written as $[17]$

$$
\Gamma_{\mathrm{L}, \mathrm{UV}}^{1 \ell}\left[\phi_{\mathrm{cl}}\right]=\frac{i}{2} \log \operatorname{det}\left(\mathcal{Q}_{11}-\mathcal{Q}_{12} \mathcal{Q}_{22}^{-1} \mathcal{Q}_{21}\right)+\frac{i}{2} \log \operatorname{det} \mathcal{Q}_{22} .
$$

Using similar arguments for the Lagrangian of the EFT, $\mathcal{L}_{\mathrm{EFT}}[\phi]$, which only depends on the light fields, the generator of 1PI Green's functions in the EFT can be calculated at one-loop as

$$
\Gamma_{\mathrm{EFT}}^{1 \ell}\left[\phi_{\mathrm{cl}}\right]=\int \mathrm{d}^{d} x \mathcal{L}_{\mathrm{EFT}}^{1 \ell}\left[\phi_{\mathrm{cl}}\right]+\frac{i}{2} \log \operatorname{det}\left(-\frac{\delta^{2} \mathcal{L}_{\mathrm{EFT}}^{\mathrm{tree}}}{\delta \phi \delta \phi}\left[\phi_{\mathrm{cl}}\right]\right),
$$

where $\mathcal{L}_{\mathrm{EFT}}^{1 \ell}$ is the effective Lagrangian whose couplings are given by the one-loop heavy or heavy/light field contributions. The second term contains one-loop contributions constructed from the tree-level part of the effective Lagrangian $\mathcal{L}_{\text {EFT }}^{\text {tree }}$. The matching condition (2.1) then implies

$$
\begin{aligned}
\int \mathrm{d}^{d} x \mathcal{L}_{\mathrm{EFT}}^{1 \ell}[\phi]= & \frac{i}{2} \log \operatorname{det}\left(\mathcal{Q}_{11}-\mathcal{Q}_{12} \mathcal{Q}_{22}^{-1} \mathcal{Q}_{21}\right)+\frac{i}{2} \log \operatorname{det} \mathcal{Q}_{22} \\
& -\frac{i}{2} \log \operatorname{det}\left(-\frac{\delta^{2} \mathcal{L}_{\mathrm{EFT}}^{\mathrm{tree}}}{\delta \phi \delta \phi}\left[\phi_{\mathrm{cl}}\right]\right) .
\end{aligned}
$$

The functional determinants can be calculated using the relation $\log \operatorname{det} A=\operatorname{Tr} \log A$ and then calculating the trace. This includes a trace in the Hilbert space as constructed in [27]. It is convenient to calculate this trace in position space and insert the identity in terms of a complete set of momentum eigenstates. The calculation then involves an integral over the 
four-momentum, and expansion by regions $[28,29]$ can be applied to the integrals $[17,18]$. It can then be shown [18] that

$$
\mathcal{L}_{\mathrm{EFT}}^{1 \ell}[\phi]=\left.\frac{i}{2} \int \frac{\mathrm{d}^{d} q}{(2 \pi)^{d}} \operatorname{tr} \log \left(\mathcal{Q}_{11}-\mathcal{Q}_{12} \mathcal{Q}_{22}^{-1} \mathcal{Q}_{21}\right)\right|_{\text {hard }} ^{P \rightarrow P-q},
$$

where the final result is given by the hard part of the integrals, i.e. the part for which the integrands can be expanded in the region $\left|q^{2}\right| \sim M^{2} \gg m^{2}$ and where $P_{\mu}=i D_{\mu}$ with $D_{\mu}$ being the gauge-covariant derivative. In (2.13) the trace over the Hilbert space has already been performed and "tr" designates a trace over all indices. To derive the currently known form of the purely scalar UOLEA $[8,9]$ from $(2.13)$, one expands the logarithm in a power series, which is evaluated up to terms giving rise to operators of mass dimension 6 and calculates the corresponding coefficients arising from the momentum integral. In order to keep gauge-invariance manifest in the resulting $\mathcal{L}_{\mathrm{EFT}}^{1 \ell}$ a covariant derivative expansion $[11,12]$ is used, where $P^{\mu}$ is kept as a whole and not split into a partial derivative and gauge fields.

\subsection{Fermionic contributions to the UOLEA}

In this section we consider a more general theory which contains both scalar and fermionic fields and calculate their contributions to the UOLEA. ${ }^{2}$ This extends the results provided in [9] by including contributions to the matching from loops containing both scalars and fermions as well as contributions from purely fermionic loops. The latter are partially contained in the results of [9] since they can be computed by squaring the purely fermionic trace. However, in this approach contributions are missed whenever the interaction terms among fermions contain gamma matrices. These terms would be classified as terms with open covariant derivatives in the language used in [9]. In our treatment no assumptions are made about the spin structure of the fermionic interactions. In principle, the calculation can be performed using the method of covariant diagrams introduced in [18], however, the calculation is presented starting from first principles for the following reason. There is some freedom in choosing the degrees of freedom to integrate over in the path integral. For complex scalar fields, for example, these can be the real and imaginary parts of the field. Alternatively one can choose the field and its conjugate as independent degrees of freedom. For fermions similar choices can be made. The explicit form of the fluctuation operator and the transformations necessary to bring the Gaussian path integral into a form where it can be trivially performed depend on this choice. To reduce the number of these transformations we use a formalism where Dirac and Majorana fermions are treated together in one multiplet in the diagonalization step. Our formalism has the additional advantage, that the resulting expressions are more compact compared to the case when Dirac and Majorana fermions are treated separately. In the following we will present our formalism in detail and introduce the notation of the final result.

As mentioned above, there is some freedom in the choice of degrees of freedom to be integrated over. In order to treat real and complex scalar fields on the same footing

\footnotetext{
${ }^{2}$ As discussed in [18] and section 3.3, our final expression for the UOLEA can also be used in a more general setting, including, for example, massive vector fields.
} 


\begin{tabular}{|cll|}
\hline Multiplet & Components & Description \\
\hline$\Xi$ & $\left(\Omega, \Omega^{C}, \Lambda\right)^{T}$ & $\begin{array}{l}\Omega, \Omega^{C}: \text { heavy Dirac fermions } \\
\Lambda: \text { heavy Majorana fermions }\end{array}$ \\
\hline$\Phi$ & $\left(\Sigma, \Sigma^{*}, \Theta\right)^{T}$ & $\begin{array}{l}\Sigma, \Sigma^{*}: \text { heavy complex scalars } \\
\Theta: \text { heavy real scalars }\end{array}$ \\
\hline$\xi$ & $\left(\omega, \omega^{C}, \lambda\right)^{T}$ & $\begin{array}{l}\omega, \omega^{C}: \text { light Dirac fermions } \\
\lambda: \text { light Majorana fermions }\end{array}$ \\
\hline \multirow{2}{*}{$\begin{array}{c}\left(\sigma, \sigma^{*}, \theta\right)^{T} \\
\end{array}$} & $\begin{array}{l}\sigma, \sigma^{*}: \text { light complex scalars } \\
\theta: \text { light real scalars }\end{array}$ \\
\hline
\end{tabular}

Table 1. Contents of the different multiplets appearing in the calculation.

one could split all complex fields into a real part and an imaginary part and perform the calculation using these as the fundamental fields. However, for scalars it is often desirable to maintain the complex fields as they might have some physical interpretation in the effective theory. We therefore use the field and its complex conjugate as independent degrees of freedom. Similarly, in order to treat Dirac and Majorana fermions simultaneously without diagonalizing the fluctuation operator among these it is convenient to treat any Dirac fermion and its charge conjugate as independent degrees of freedom. We collect all light and heavy scalars into the multiplets $\phi$ and $\Phi$, respectively, and all light and heavy fermions into the multiplets $\xi$ and $\Xi$, respectively, see table 1 . The charge conjugate of the Dirac spinor $\Omega$ is denoted as $\Omega^{C}=\mathcal{C} \bar{\Omega}^{T}$, with $\mathcal{C}$ being the charge conjugation matrix. Similarly, we define for a light Dirac spinor $\omega, \omega^{C}=\mathcal{C} \bar{\omega}^{T}$. With these definitions we may write the second variation of the Lagrangian as follows

$$
\begin{aligned}
\delta^{2} \mathcal{L}= & \delta^{2} \mathcal{L}_{\mathrm{S}}+\frac{1}{2} \delta \Xi^{T} \boldsymbol{\Delta}_{\Xi} \delta \Xi-\frac{1}{2} \delta \Xi^{T} \tilde{\mathbf{X}}_{\Xi \Phi} \delta \Phi+\frac{1}{2} \delta \Phi^{T} \tilde{\mathbf{X}}_{\Phi \Xi} \delta \Xi-\frac{1}{2} \delta \Xi^{T} \tilde{\mathbf{X}}_{\Xi \phi} \delta \phi \\
& +\frac{1}{2} \delta \phi^{T} \tilde{\mathbf{X}}_{\phi \Xi} \delta \Xi+\frac{1}{2} \delta \xi^{T} \tilde{\mathbf{X}}_{\xi \Xi} \delta \Xi+\frac{1}{2} \delta \Xi^{T} \tilde{\mathbf{X}}_{\Xi \xi} \delta \xi+\frac{1}{2} \delta \xi^{T} \boldsymbol{\Delta}_{\xi} \delta \xi \\
& -\frac{1}{2} \delta \xi^{T} \tilde{\mathbf{X}}_{\xi \Phi} \delta \Phi+\frac{1}{2} \delta \Phi^{T} \tilde{\mathbf{X}}_{\Phi \xi} \delta \xi-\frac{1}{2} \delta \xi^{T} \tilde{\mathbf{X}}_{\xi \phi} \delta \phi+\frac{1}{2} \delta \phi^{T} \tilde{\mathbf{X}}_{\phi \xi} \delta \xi
\end{aligned}
$$

where the pure scalar part is given by

$$
\delta^{2} \mathcal{L}_{\mathrm{S}}=-\frac{1}{2} \delta \Phi^{T} \boldsymbol{\Delta}_{\Phi} \delta \Phi-\frac{1}{2} \delta \phi^{T} \boldsymbol{\Delta}_{\phi} \delta \phi-\frac{1}{2} \delta \Phi^{T} \tilde{\mathbf{X}}_{\Phi \phi} \delta \phi-\frac{1}{2} \delta \phi^{T} \tilde{\mathbf{X}}_{\phi \Phi} \delta \Phi .
$$

In eqs. (2.14) and (2.15) we introduced the following abbreviations:

$$
\begin{aligned}
\boldsymbol{\Delta}_{\Xi} & =\left(\begin{array}{ccc}
X_{\Omega \Omega} & \mathcal{C}\left(\not P_{\Omega^{C}}-M_{\Omega}+\mathcal{C}^{-1} X_{\Omega \bar{\Omega}} \mathcal{C}^{-1}\right) & X_{\Omega \Lambda} \\
\mathcal{C}\left(\not P_{\Omega}-M_{\Omega}+X_{\bar{\Omega} \Omega}\right) & \mathcal{C} X_{\bar{\Omega} \bar{\Omega}} \mathcal{C}^{-1} & \mathcal{C} X_{\bar{\Omega} \Lambda} \\
X_{\Lambda \Omega} & X_{\Lambda \bar{\Omega}} \mathcal{C}^{-1} & \mathcal{C}\left(\not P_{\Lambda}-M_{\Lambda}+\mathcal{C}^{-1} X_{\Lambda \Lambda}\right)
\end{array}\right), \\
\tilde{\mathbf{X}}_{\Xi \Phi} & =\left(\begin{array}{ccc}
X_{\Omega \Sigma} & X_{\Omega \Sigma^{*}} & X_{\Omega \Theta} \\
\mathcal{C} X_{\bar{\Omega} \Sigma} & \mathcal{C} X_{\bar{\Omega} \Sigma^{*}} & \mathcal{C} X_{\bar{\Omega} \Theta} \\
X_{\Lambda \Sigma} & X_{\Lambda \Sigma^{*}} & X_{\Lambda \Theta}
\end{array}\right),
\end{aligned}
$$




$$
\begin{aligned}
\tilde{\mathbf{X}}_{\Phi \Xi}= & \left(\begin{array}{ccc}
X_{\Sigma \Omega} & X_{\Sigma \bar{\Omega}} \mathcal{C}^{-1} & X_{\Sigma \Lambda} \\
X_{\Sigma^{*} \Omega} & X_{\Sigma^{*} \bar{\Omega}} \mathcal{C}^{-1} & X_{\Sigma^{*} \Lambda} \\
X_{\Theta \Omega} & X_{\Theta \bar{\Omega}} \mathcal{C}^{-1} & X_{\Theta \Lambda}
\end{array}\right), \\
\tilde{\mathbf{X}}_{\Xi \xi} & =\left(\begin{array}{ccc}
X_{\Omega \omega} & X_{\Omega \bar{\omega}} \mathcal{C}^{-1} & X_{\Omega \lambda} \\
\mathcal{C} X_{\bar{\Omega} \omega} & \mathcal{C} X_{\bar{\Omega} \bar{\omega}} \mathcal{C}^{-1} & \mathcal{C} X_{\bar{\Omega} \lambda} \\
X_{\Lambda \omega} & X_{\Lambda \bar{\omega}} \mathcal{C}^{-1} & X_{\Lambda \lambda}
\end{array}\right), \\
\boldsymbol{\Delta}_{\Phi} & =\left(\begin{array}{ccc}
-P_{\Sigma}^{2}+M_{\Sigma \Sigma}^{2}+X_{\Sigma^{*} \Sigma} & -P_{\Sigma^{*}}^{2}+M_{\Sigma}^{2}+X_{\Sigma \Sigma^{*}} & X_{\Sigma \Theta} \\
X_{\Theta \Sigma} & X_{\Sigma^{*} \Sigma^{*}} & X_{\Sigma^{*} \Theta} \\
- & X_{\Theta \Sigma^{*}} & -P_{\Theta}^{2}+M_{\Theta}^{2}+X_{\Theta \Theta}
\end{array}\right),
\end{aligned}
$$

with similar definitions for $\Phi \rightarrow \phi$ and $\Xi \rightarrow \xi$. Here $P^{\mu} \equiv i D^{\mu}$, with $D^{\mu}$ being the gaugecovariant derivative, is a matrix diagonal in field space for which the subscript indicates which gauge group generators are to be used. Furthermore we have defined

$$
\left(X_{A B}\right)_{i j} \equiv-\frac{\delta^{2} \mathcal{L}_{\mathrm{UV}, \text { int }}}{\delta A_{i} \delta B_{j}}
$$

where $\mathcal{L}_{\mathrm{UV} \text {,int }}$ is the interaction Lagrangian of the UV theory and $A$ and $B$ designate arbitrary (scalar or fermionic) fields, if not stated otherwise. Here the indices $i$ and $j$ collectively denote all of the indices carried by the fields $A$ and $B$. It shall be noted that if $P_{\Omega}^{\mu}$ contains generators $T_{r}^{a}$ of a representation $r$, then $P_{\Omega^{C}}^{\mu}$ contains the generators of the conjugate representation $\bar{r}$, denoted by $T_{\bar{r}}^{a}$. The same holds for the generators contained in $P_{\Sigma}^{\mu}$ and $P_{\Sigma^{*}}^{\mu}$. Note also that $(2.15)$ is in principle equivalent to the quadratic term in (2.7) with the difference being that in (2.7) all scalar fields are assumed to be real, while in (2.15) complex and real fields are separate. The different signs in the fermionic terms in (2.14) result from using the anti-commutation relation between fermions and derivatives w.r.t. fermions.

Before proceeding it is convenient to define

$$
\tilde{\mathbb{1}} \equiv\left(\begin{array}{lll}
0 & \mathbb{1} & 0 \\
\mathbb{1} & 0 & 0 \\
0 & 0 & \mathbb{1}
\end{array}\right),
$$

and rewrite (2.16) as

$$
\boldsymbol{\Delta}_{\Xi}=\mathcal{C} \tilde{\mathbb{1}}\left(\not P-M_{\Xi}\right)+\tilde{\mathbf{X}}_{\Xi \Xi}
$$

where

$$
\begin{aligned}
\not P-M_{\Xi} & =\left(\begin{array}{ccc}
\not P_{\Omega}-M_{\Omega} & 0 & 0 \\
0 & \not_{\Omega^{C}}-M_{\Omega} & 0 \\
0 & 0 & \not P_{\Lambda}-M_{\Lambda}
\end{array}\right), \\
\tilde{\mathbf{X}}_{\Xi \Xi} & =\left(\begin{array}{ccc}
X_{\omega \omega} & X_{\Omega \bar{\Omega}} \mathcal{C}^{-1} & X_{\Omega \Lambda} \\
\mathcal{C} X_{\bar{\Omega} \Omega} & \mathcal{C} X_{\bar{\omega} \bar{\omega}} \mathcal{C}^{-1} & \mathcal{C} X_{\bar{\Omega} \Lambda} \\
X_{\Lambda \Omega} & X_{\Lambda \bar{\Omega}} \mathcal{C}^{-1} & X_{\Lambda \Lambda}
\end{array}\right)
\end{aligned}
$$


We rewrite (2.20) in a similar way as

$$
\boldsymbol{\Delta}_{\Phi}=\tilde{\mathbb{1}}\left(-P^{2}+M_{\Phi}^{2}\right)+\tilde{\mathbf{X}}_{\Phi \Phi}
$$

with

$$
\begin{aligned}
-P^{2}+M_{\Phi}^{2} & =\left(\begin{array}{ccc}
-P_{\Sigma}^{2}+M_{\Sigma}^{2} & 0 & 0 \\
0 & -P_{\Sigma^{*}}^{2}+M_{\Sigma^{*}}^{2} & 0 \\
0 & 0 & -P_{\Theta}^{2}+M_{\Theta}^{2}
\end{array}\right), \\
\tilde{\mathbf{X}}_{\Phi \Phi} & =\left(\begin{array}{ccc}
X_{\Sigma \Sigma} & X_{\Sigma \Sigma^{*}} & X_{\Sigma \Theta} \\
X_{\Sigma^{*} \Sigma} & X_{\Sigma^{*} \Sigma^{*}} & X_{\Sigma^{*} \Theta} \\
X_{\Theta \Sigma} & X_{\Theta \Sigma^{*}} & X_{\Theta \Theta}
\end{array}\right) .
\end{aligned}
$$

The calculation now proceeds by diagonalizing the quadratic variation in terms of statistics in order to be able to perform the (Gaussian) path integral. We first eliminate terms that mix scalar fluctuations and fluctuations of light fermions $\xi$ by rewriting the second variation as

$$
\begin{aligned}
\delta^{2} \mathcal{L}_{\xi}= & \frac{1}{2} \delta \xi^{T} \tilde{\mathbf{X}}_{\xi \Xi} \delta \Xi+\frac{1}{2} \delta \Xi^{T} \tilde{\mathbf{X}}_{\Xi \xi} \delta \xi+\frac{1}{2} \delta \xi^{T} \boldsymbol{\Delta}_{\xi} \delta \xi-\frac{1}{2} \delta \xi^{T} \tilde{\mathbf{X}}_{\xi \Phi} \delta \Phi+\frac{1}{2} \delta \Phi^{T} \tilde{\mathbf{X}}_{\Phi \xi} \delta \xi \\
& -\frac{1}{2} \delta \xi^{T} \tilde{\mathbf{X}}_{\xi \phi} \delta \phi+\frac{1}{2} \delta \phi^{T} \tilde{\mathbf{X}}_{\phi \xi} \delta \xi \\
= & \frac{1}{2}\left(\delta \xi^{T}+\left[\delta \Xi^{T} \tilde{\mathbf{X}}_{\Xi \xi}+\delta \Phi^{T} \tilde{\mathbf{X}}_{\Phi \xi}+\delta \phi^{T} \tilde{\mathbf{X}}_{\phi \xi}\right] \overleftarrow{\boldsymbol{\Delta}}_{\xi}^{-1}\right) \boldsymbol{\Delta}_{\xi} \\
& \times\left(\delta \xi+\boldsymbol{\Delta}_{\xi}^{-1}\left[\tilde{\mathbf{X}}_{\xi \Xi} \delta \Xi-\tilde{\mathbf{X}}_{\xi \Phi} \delta \Phi-\tilde{\mathbf{X}}_{\xi \phi} \delta \phi\right]\right) \\
& -\frac{1}{2}\left[\delta \Xi^{T} \tilde{\mathbf{X}}_{\Xi \xi}+\delta \Phi^{T} \tilde{\mathbf{X}}_{\Phi \xi}+\delta \phi^{T} \tilde{\mathbf{X}}_{\phi \xi}\right] \boldsymbol{\Delta}_{\xi}^{-1}\left[\tilde{\mathbf{X}}_{\xi \Xi} \delta \Xi-\tilde{\mathbf{X}}_{\xi \Phi} \delta \Phi-\tilde{\mathbf{X}}_{\xi \phi} \delta \phi\right]
\end{aligned}
$$

In the last step we have introduced $\boldsymbol{\Delta}_{\xi}^{-1}$, which is the matrix-valued Green's function of $\boldsymbol{\Delta}_{\xi}$. The occurring matrix multiplication also implies an integration, that is

$$
\begin{aligned}
\left(\boldsymbol { \Delta } _ { \xi } ^ { - 1 } \left[\tilde{\mathbf{X}}_{\xi \Xi} \delta \Xi\right.\right. & \left.\left.-\tilde{\mathbf{X}}_{\xi \Phi} \delta \Phi-\tilde{\mathbf{X}}_{\xi \phi} \delta \phi\right]\right)(x) \\
& \equiv \int \mathrm{d}^{d} y \boldsymbol{\Delta}_{\xi}^{-1}(x, y)\left[\tilde{\mathbf{X}}_{\xi \Xi}(y) \delta \Xi(y)-\tilde{\mathbf{X}}_{\xi \Phi}(y) \delta \Phi(y)-\tilde{\mathbf{X}}_{\xi \phi}(y) \delta \phi(y)\right] .
\end{aligned}
$$

Similar to $\boldsymbol{\Delta}_{\xi}^{-1}$ we define $\overleftarrow{\boldsymbol{\Delta}}_{\xi}^{-1}$ in such a way that

$$
\int \mathrm{d}^{d} y f(y) \overleftarrow{\Delta}_{\xi}^{-1}(y, x) \overleftarrow{\Delta}_{\xi}(x)=f(x)
$$

where $\overleftarrow{\Delta}_{\xi}(x)=-\overleftarrow{\not}-M_{\xi}$. Next, we shift the light fermion field as

$$
\begin{aligned}
\delta \xi^{\prime} & =\delta \xi+\boldsymbol{\Delta}_{\xi}^{-1}\left[\tilde{\mathbf{X}}_{\xi \Xi} \delta \Xi-\tilde{\mathbf{X}}_{\xi \Phi} \delta \Phi-\tilde{\mathbf{X}}_{\xi \phi} \delta \phi\right] \\
\delta \xi^{\prime T} & =\delta \xi^{T}+\left[\delta \Xi^{T} \tilde{\mathbf{X}}_{\Xi \xi}+\delta \Phi^{T} \tilde{\mathbf{X}}_{\Phi \xi}+\delta \phi^{T} \tilde{\mathbf{X}}_{\phi \xi}\right] \overleftarrow{\Delta}_{\xi}^{-1}
\end{aligned}
$$

under which the path integral measure is invariant. Since $\xi$ is a multiplet of Majoranalike spinors, the two shifts (2.33) and (2.34) are not independent. The required relation 
between the two shifts is proven in appendix A. After the shifts have been performed we arrive at

$$
\begin{aligned}
\delta^{2} \mathcal{L}_{\xi}= & \frac{1}{2} \delta \xi^{T} \boldsymbol{\Delta}_{\xi} \delta \xi^{\prime}-\frac{1}{2} \delta \Xi^{T} \tilde{\mathbf{X}}_{\Xi \xi} \boldsymbol{\Delta}_{\xi}^{-1} \tilde{\mathbf{X}}_{\xi \Xi} \delta \Xi+\frac{1}{2} \delta \Xi^{T} \tilde{\mathbf{X}}_{\Xi \xi} \boldsymbol{\Delta}_{\xi}^{-1} \tilde{\mathbf{X}}_{\xi \Phi} \delta \Phi \\
& +\frac{1}{2} \delta \Xi^{T} \tilde{\mathbf{X}}_{\Xi \xi} \boldsymbol{\Delta}_{\xi}^{-1} \tilde{\mathbf{X}}_{\xi \phi} \delta \phi+\frac{1}{2} \delta \Phi^{T} \tilde{\mathbf{X}}_{\Phi \xi} \boldsymbol{\Delta}_{\xi}^{-1} \tilde{\mathbf{X}}_{\xi \Xi} \delta \Xi-\frac{1}{2} \delta \Phi^{T} \tilde{\mathbf{X}}_{\Phi \xi} \boldsymbol{\Delta}_{\xi}^{-1} \tilde{\mathbf{X}}_{\xi \Phi} \delta \Phi \\
& -\frac{1}{2} \delta \Phi^{T} \tilde{\mathbf{X}}_{\Phi \xi} \boldsymbol{\Delta}_{\xi}^{-1} \tilde{\mathbf{X}}_{\xi \phi} \delta \phi+\frac{1}{2} \delta \phi^{T} \tilde{\mathbf{X}}_{\phi \xi} \boldsymbol{\Delta}_{\xi}^{-1} \tilde{\mathbf{X}}_{\xi \Xi} \delta \Xi-\frac{1}{2} \delta \phi^{T} \tilde{\mathbf{X}}_{\phi \xi} \boldsymbol{\Delta}_{\xi}^{-1} \tilde{\mathbf{X}}_{\xi \Phi} \delta \Phi \\
& -\frac{1}{2} \delta \phi^{T} \tilde{\mathbf{X}}_{\phi \xi} \boldsymbol{\Delta}_{\xi}^{-1} \tilde{\mathbf{X}}_{\xi \phi} \delta \phi
\end{aligned}
$$

We proceed by eliminating terms that mix scalar fluctuations and fluctuations of heavy fermions $\Xi$. It is convenient to first introduce

$$
\begin{aligned}
\overline{\mathbf{X}}_{A B} & \equiv \tilde{\mathbf{X}}_{A B}-\tilde{\mathbf{X}}_{A \xi} \boldsymbol{\Delta}_{\xi}^{-1} \tilde{\mathbf{X}}_{\xi B} \\
\overline{\boldsymbol{\Delta}}_{A} & \equiv \boldsymbol{\Delta}_{A}-\tilde{\mathbf{X}}_{A \xi} \boldsymbol{\Delta}_{\xi}^{-1} \tilde{\mathbf{X}}_{\xi A}
\end{aligned}
$$

and write the second variation as

$$
\begin{aligned}
\delta^{2} \mathcal{L}= & \delta^{2} \overline{\mathcal{L}}_{\mathrm{S}}+\frac{1}{2} \delta \Xi^{T} \overline{\boldsymbol{\Delta}}_{\Xi} \delta \Xi-\frac{1}{2} \delta \Xi^{T} \overline{\mathbf{X}}_{\Xi \Phi} \delta \Phi+\frac{1}{2} \delta \Phi^{T} \overline{\mathbf{X}}_{\Phi \Xi \delta \Xi} \\
& -\frac{1}{2} \delta \Xi^{T} \overline{\mathbf{X}}_{\Xi \phi} \delta \phi+\frac{1}{2} \delta \phi^{T} \overline{\mathbf{X}}_{\phi \Xi} \delta \Xi .
\end{aligned}
$$

In (2.38) the first term on the r.h.s., $\delta^{2} \overline{\mathcal{L}}_{\mathrm{S}}$, is obtained by replacing $\tilde{\mathbf{X}}_{A B}$ and $\boldsymbol{\Delta}_{A}$ in $\delta^{2} \mathcal{L}_{\mathrm{S}}$ via the relations (2.36)-(2.37). By shifting the $\delta \Xi$ in a similar way,

$$
\begin{aligned}
\delta \Xi^{\prime} & =\delta \Xi-\overline{\boldsymbol{\Delta}}_{\Xi}^{-1}\left[\overline{\mathbf{X}}_{\Xi \Phi} \delta \Phi+\overline{\mathbf{X}}_{\Xi \phi} \delta \phi\right] \\
\delta \Xi^{\prime T} & =\delta \Xi^{T}+\left[\delta \Phi^{T} \overline{\mathbf{X}}_{\Phi \Xi}+\delta \phi^{T} \overline{\mathbf{X}}_{\phi \Xi}\right] \overleftarrow{\bar{\Delta}}_{\Xi}^{-1}
\end{aligned}
$$

one finds

$$
\begin{aligned}
& \delta^{2} \mathcal{L}=-\frac{1}{2} \delta \Phi^{T}\left(\overline{\boldsymbol{\Delta}}_{\Phi}-\overline{\mathbf{X}}_{\Phi \Xi} \overline{\boldsymbol{\Delta}}_{\Xi}^{-1} \overline{\mathbf{X}}_{\Xi \Phi}\right) \delta \Phi-\frac{1}{2} \delta \phi^{T}\left(\overline{\boldsymbol{\Delta}}_{\phi}-\overline{\mathbf{X}}_{\phi \Xi} \overline{\boldsymbol{\Delta}}_{\Xi}^{-1} \overline{\mathbf{X}}_{\Xi \phi}\right) \delta \phi \\
& -\frac{1}{2} \delta \Phi^{T}\left(\overline{\mathbf{X}}_{\Phi \phi}-\overline{\mathbf{X}}_{\Phi \Xi} \overline{\boldsymbol{\Delta}}_{\Xi}^{-1} \overline{\mathbf{X}}_{\Xi \phi}\right) \delta \phi \\
& -\frac{1}{2} \delta \phi^{T}\left(\overline{\mathbf{X}}_{\phi \Phi}-\overline{\mathbf{X}}_{\phi \Xi} \overline{\boldsymbol{\Delta}}_{\Xi}^{-1} \overline{\mathbf{X}}_{\Xi \Phi}\right) \delta \Phi+\frac{1}{2} \delta \xi^{T} \boldsymbol{\Delta}_{\xi} \delta \xi^{\prime}+\frac{1}{2} \delta \Xi^{\prime T} \overline{\boldsymbol{\Delta}}_{\Xi} \delta \Xi^{\prime} \\
& =-\frac{1}{2}\left(\begin{array}{ll}
\delta \Phi^{T} & \delta \phi^{T}
\end{array}\right)\left(\begin{array}{cc}
\overline{\boldsymbol{\Delta}}_{\Phi}-\overline{\mathbf{X}}_{\Phi \Xi} \overline{\boldsymbol{\Delta}}_{\Xi}^{-1} \overline{\mathbf{X}}_{\Xi \Phi} & \overline{\mathbf{X}}_{\Phi \phi}-\overline{\mathbf{X}}_{\Phi \Xi \overline{\boldsymbol{\Delta}}} \bar{\Xi}_{\Xi}^{-1} \overline{\mathbf{X}}_{\Xi \phi} \\
\overline{\mathbf{X}}_{\phi \Phi}-\overline{\mathbf{X}}_{\phi \Xi} \overline{\boldsymbol{\Delta}}_{\Xi}^{-1} \overline{\mathbf{X}}_{\Xi \Phi} & \overline{\boldsymbol{\Delta}}_{\phi}-\overline{\mathbf{X}}_{\phi \Xi} \overline{\boldsymbol{\Delta}}_{\Xi}^{-1} \overline{\mathbf{X}}_{\Xi \phi}
\end{array}\right)\left(\begin{array}{c}
\delta \Phi \\
\delta \phi
\end{array}\right) \\
& +\frac{1}{2} \delta \xi^{\prime T} \boldsymbol{\Delta}_{\xi} \delta \xi^{\prime}+\frac{1}{2} \delta \Xi^{\prime T} \overline{\boldsymbol{\Delta}}_{\Xi} \delta \Xi^{\prime} \\
& \equiv-\frac{1}{2}\left(\begin{array}{ll}
\delta \Phi^{T} & \delta \phi^{T}
\end{array}\right) \mathcal{Q}_{\mathrm{S}}\left(\begin{array}{l}
\delta \Phi \\
\delta \phi
\end{array}\right)+\frac{1}{2} \delta \xi^{\prime T} \boldsymbol{\Delta}_{\xi} \delta \xi^{\prime}+\frac{1}{2} \delta \Xi^{\prime T} \overline{\boldsymbol{\Delta}}_{\Xi} \delta \Xi^{\prime} \\
& \equiv \delta^{2} \mathcal{L}_{\mathrm{SF}}+\delta^{2} \mathcal{L}_{\mathrm{F}}
\end{aligned}
$$


with

$$
\begin{aligned}
\delta^{2} \mathcal{L}_{\mathrm{SF}} & =-\frac{1}{2}\left(\begin{array}{ll}
\delta \Phi^{T} & \delta \phi^{T}
\end{array}\right) \mathcal{Q}_{\mathrm{S}}\left(\begin{array}{c}
\delta \Phi \\
\delta \phi
\end{array}\right) \\
\delta^{2} \mathcal{L}_{\mathrm{F}} & =\frac{1}{2} \delta \xi^{\prime T} \boldsymbol{\Delta}_{\xi} \delta \xi^{\prime}+\frac{1}{2} \delta \Xi^{\prime T} \overline{\boldsymbol{\Delta}}_{\Xi} \delta \Xi^{\prime}
\end{aligned}
$$

At this point there are no terms including both a scalar and a fermionic fluctuation and the path integrals over scalars and fermions can be performed separately. As has been pointed out in [17] it is convenient to diagonalize the scalar part such that

$$
\mathcal{Q}_{\mathrm{S}}=\left(\begin{array}{cc}
\hat{\boldsymbol{\Delta}}_{\Phi}-\hat{\mathbf{X}}_{\Phi \phi} \hat{\boldsymbol{\Delta}}_{\phi}^{-1} \hat{\mathbf{X}}_{\phi \Phi} & 0 \\
0 & \hat{\boldsymbol{\Delta}}_{\phi}
\end{array}\right),
$$

where

$$
\begin{aligned}
\hat{\boldsymbol{\Delta}}_{A} & =\overline{\boldsymbol{\Delta}}_{A}-\overline{\mathbf{X}}_{A \Xi} \overline{\boldsymbol{\Delta}}_{\Xi}^{-1} \overline{\mathbf{X}}_{\Xi A}, \\
\hat{\mathbf{X}}_{A B} & =\overline{\mathbf{X}}_{A B}-\overline{\mathbf{X}}_{A \Xi} \overline{\boldsymbol{\Delta}}_{\Xi}^{-1} \overline{\mathbf{X}}_{\Xi B},
\end{aligned}
$$

with $A, B \in\{\phi, \Phi\}$. The contribution from this mixed scalar/fermionic part to the effective action is then given by

$$
\mathcal{L}_{\mathrm{EFT}, \mathrm{SF}}^{1 \ell}=\left.\frac{i}{2} \int \frac{\mathrm{d}^{d} q}{(2 \pi)^{d}}\left[\operatorname{tr} \log \left(\hat{\boldsymbol{\Delta}}_{\Phi}-\hat{\mathbf{X}}_{\Phi \phi} \hat{\boldsymbol{\Delta}}_{\phi}^{-1} \hat{\mathbf{X}}_{\phi \Phi}\right)+\operatorname{tr} \log \hat{\boldsymbol{\Delta}}_{\phi}\right]\right|_{\text {hard }} ^{P \rightarrow P-q}
$$

and it can be calculated using a covariant derivative expansion as outlined in e.g. [18]. However, care has to be taken since $\hat{\boldsymbol{\Delta}}_{\phi}$ contains contributions from heavy fermions and hence does not vanish completely in the hard region of the momentum integration. The corresponding contributions can be calculated by using

$$
\log \operatorname{det}\left(\overline{\boldsymbol{\Delta}}_{\phi}-\overline{\mathbf{X}}_{\phi \Xi} \overline{\boldsymbol{\Delta}}_{\Xi}^{-1} \overline{\mathbf{X}}_{\Xi \phi}\right)=\log \operatorname{det}\left(\overline{\boldsymbol{\Delta}}_{\phi}\right)+\log \operatorname{det}\left(\mathbb{1}-\overline{\boldsymbol{\Delta}}_{\phi}^{-1} \overline{\mathbf{X}}_{\phi \Xi} \overline{\boldsymbol{\Delta}}_{\Xi}^{-1} \overline{\mathbf{X}}_{\Xi \phi}\right),
$$

where the first term on the right hand side vanishes in the hard region as it only contains contributions from light fields.

Since a lot of terms are generated when re-expressing the hatted and barred quantities in terms of the quantities arising in the original variation (2.35) we abstain from writing out the result explicitly. It is, however, useful to consider the expansion of the hatted operators in order to understand the ingredients entering the final result. In particular we will show that it is possible to absorb all explicit factors of $\tilde{\mathbb{1}}$ and $\mathcal{C}$ by appropriate re-definitions of $\tilde{\mathbf{X}}_{A B}$. In order to achieve that we first expand $\left(\boldsymbol{\Delta}_{\xi}^{-1}\right)_{P_{\mu} \rightarrow P_{\mu}-q_{\mu}} \equiv \boldsymbol{\Delta}_{\xi}^{-1}(q)$ as

$$
\begin{aligned}
\boldsymbol{\Delta}_{\xi}^{-1}(q) & =\left[\mathcal{C} \tilde{\mathbb{1}}\left(\not P-\not q-M_{\xi}\right)+\tilde{\mathbf{X}}_{\xi \xi}\right]^{-1} \\
& =\left[\mathbb{1}-\left(-\not q-M_{\xi}\right)^{-1} \tilde{\mathbb{1}} \mathcal{C}^{-1}\left(-\mathcal{C} \tilde{\mathbb{1}} \not P-\tilde{\mathbf{X}}_{\xi \xi}\right)\right]\left(-\not q-M_{\xi}\right)^{-1} \tilde{\mathbb{1}} \mathcal{C}^{-1} \\
& =\sum_{n=0}^{\infty}\left[\left(-\not q-M_{\xi}\right)^{-1} \tilde{\mathbb{1}} \mathcal{C}^{-1}\left(-\mathcal{C} \tilde{\mathbb{1}} \not P-\tilde{\mathbf{X}}_{\xi \xi}\right)\right]^{n}\left(-\not q-M_{\xi}\right)^{-1} \tilde{\mathbb{1}} \mathcal{C}^{-1} \\
& =\sum_{n=0}^{\infty}\left[\left(-\not q-M_{\xi}\right)^{-1}\left(-\not P-\mathbf{X}_{\xi \xi}\right)\right]^{n}\left(-\not q-M_{\xi}\right)^{-1} \tilde{\mathbb{1}} \mathcal{C}^{-1},
\end{aligned}
$$


where we defined

$$
\mathbf{X}_{\xi \xi} \equiv \tilde{\mathbb{1}} \mathcal{C}^{-1} \tilde{\mathbf{X}}_{\xi \xi}
$$

Then (2.36)-(2.37) become

$$
\begin{aligned}
\overline{\mathbf{X}}_{A B} & =\tilde{\mathbf{X}}_{A B}-\tilde{\mathbf{X}}_{A \xi} \sum_{n=0}^{\infty}\left[\left(-\not q-M_{\xi}\right)^{-1}\left(-\mathbf{X}_{\xi \xi}-\not p\right)\right]^{n}\left(-\not q-M_{\xi}\right)^{-1} \mathbf{X}_{\xi B} \\
\overline{\boldsymbol{\Delta}}_{A} & =\boldsymbol{\Delta}_{A}-\tilde{\mathbf{X}}_{A \xi} \sum_{n=0}^{\infty}\left[\left(-\not q-M_{\xi}\right)^{-1}\left(-\mathbf{X}_{\xi \xi}-\not p\right)\right]^{n}\left(-\not q-M_{\xi}\right)^{-1} \mathbf{X}_{\xi A}
\end{aligned}
$$

where we introduced $\mathbf{X}_{\xi B} \equiv \mathcal{C}^{-1} \tilde{1} \tilde{\mathbf{X}}_{\xi B}$. Next we consider

$$
\begin{aligned}
& \bar{\Delta}_{\Xi}^{-1}(q)=[\mathcal{C} \tilde{\mathbb{1}}(-\not q-\left.M_{\Xi}\right)+\mathcal{C} \tilde{\mathbb{1}} \not P+\tilde{\mathbf{X}}_{\Xi \Xi} \\
&\left.\quad-\tilde{\mathbf{X}}_{\Xi \xi} \sum_{n=0}^{\infty}\left[\left(-\not q-M_{\xi}\right)^{-1}\left(-\mathbf{X}_{\xi \xi}-\not p\right)\right]^{n}\left(-\not q-M_{\xi}\right)^{-1} \mathbf{X}_{\xi \Xi}\right]^{-1} \\
&=\sum_{m=0}^{\infty}\left\{\mathcal{K}_{\Xi}^{-1}\left(-\mathbf{X}_{\Xi \Xi}-\not p\right)\right. \\
&\left.\quad+\mathcal{K}_{\Xi}^{-1} \mathbf{X}_{\Xi \xi} \sum_{n=0}^{\infty}\left[\mathcal{K}_{\xi}^{-1}\left(-\mathbf{X}_{\xi \xi}-\not p\right)\right]^{n} \mathcal{K}_{\xi}^{-1} \mathbf{X}_{\xi \Xi}\right\}^{m} \mathcal{K}_{\Xi}^{-1} \mathcal{C}^{-1} \tilde{\mathbb{1}}
\end{aligned}
$$

where

$$
\begin{aligned}
\mathcal{K}_{A} & \equiv\left(-\not q-M_{A}\right), \\
\mathbf{X}_{\Xi \xi} & \equiv \mathcal{C}^{-1} \tilde{\mathbb{1}} \tilde{\mathbf{X}}_{\Xi \xi} .
\end{aligned}
$$

Note that in (2.55) and (2.60) the expressions for $\boldsymbol{\Delta}_{\xi}^{-1}$ and $\overline{\boldsymbol{\Delta}}_{\Xi}^{-1}$ contain the factor $\mathcal{C}^{-1} \tilde{\mathbb{1}}$ on the very right. This means that in the combination

$$
\begin{aligned}
\overline{\boldsymbol{\Delta}}_{\Xi}^{-1} \overline{\mathbf{X}}_{\Xi B} & =\overline{\boldsymbol{\Delta}}_{\Xi}^{-1}\left(\tilde{\mathbf{X}}_{\Xi B}-\tilde{\mathbf{X}}_{\Xi \xi} \boldsymbol{\Delta}_{\xi}^{-1} \tilde{\mathbb{1}} \mathcal{C} \mathcal{C}^{-1} \tilde{\mathbb{1}} \tilde{\mathbf{X}}_{\xi B}\right) \\
& =\overline{\boldsymbol{\Delta}}_{\Xi}^{-1} \tilde{\mathbb{1}} \mathcal{C} \mathcal{C}^{-1} \tilde{\mathbb{1}}\left(\tilde{\mathbf{X}}_{\Xi B}-\tilde{\mathbf{X}}_{\Xi \xi} \boldsymbol{\Delta}_{\xi}^{-1} \tilde{\mathbb{1}} \mathcal{C} \mathcal{C}^{-1} \tilde{\mathbb{1}} \tilde{\mathbf{X}}_{\xi B}\right) \\
& =\overline{\boldsymbol{\Delta}}_{\Xi}^{-1} \tilde{\mathbb{1}} \mathcal{C}\left(\mathbf{X}_{\Xi B}-\mathbf{X}_{\Xi \xi} \boldsymbol{\Delta}_{\xi}^{-1} \tilde{\mathbb{1}} \mathcal{C} \mathbf{X}_{\xi B}\right),
\end{aligned}
$$

all appearances of $\mathcal{C}$ and $\tilde{\mathbb{1}}$ cancel once $\overline{\boldsymbol{\Delta}}_{\Xi}^{-1}$ and $\boldsymbol{\Delta}_{\xi}^{-1}$ are inserted and $\tilde{\mathbf{X}}_{A B}$ is expressed in terms of $\mathbf{X}_{A B}$ with $\mathbf{X}_{A B}=\mathcal{C}^{-1} \tilde{\mathbb{1}} \tilde{\mathbf{X}}_{A B}$. A similar property holds for $\tilde{\mathbf{X}}_{\Phi B}$ and $\tilde{\mathbf{X}}_{\phi B}$, which only appear as $\mathbf{X}_{\Phi B}=\tilde{\mathbb{1}} \tilde{\mathbf{X}}_{\Phi B}$ and $\mathbf{X}_{\phi B}=\tilde{\mathbb{1}} \tilde{\mathbf{X}}_{\phi B}$. Hence, the result can be expressed entirely through the matrices $\mathbf{X}_{A B}$ and neither $\tilde{\mathbb{1}}$ nor $\mathcal{C}$ explicitly appears in the final operator structures.

To complete the calculation we need to compute the purely fermionic part of the second variation (2.44), which reads

$$
\delta^{2} \mathcal{L}_{\mathrm{F}}=\frac{1}{2} \delta \Xi^{\prime T} \overline{\boldsymbol{\Delta}}_{\Xi} \delta \Xi^{\prime}+\frac{1}{2} \delta \xi^{\prime T} \boldsymbol{\Delta}_{\xi} \delta \xi^{\prime}
$$


Again, we are only interested in the contribution from the hard region where the light only part $\boldsymbol{\Delta}_{\xi}$ does not contribute. Hence we only need to consider $\overline{\boldsymbol{\Delta}}_{\Xi}$. We find

$$
\begin{aligned}
\operatorname{tr} \log ( & \left.\boldsymbol{\Delta}_{\Xi}(q)-\mathbf{X}_{\Xi \xi} \Delta_{\xi}^{-1}(q) \mathbf{X}_{\xi \Xi}\right) \\
& =\operatorname{tr} \log \left(\mathcal{C} \tilde{\mathbb{1}} \mathcal{K}_{\Xi}+\mathcal{C} \tilde{\mathbb{1}} \not P+\tilde{\mathbf{X}}_{\Xi \Xi}-\mathbf{X}_{\Xi \xi} \boldsymbol{\Delta}_{\xi}^{-1}(q) \tilde{\mathbf{X}}_{\xi \Xi}\right) \\
& =\operatorname{tr} \log \left(\mathcal{C} \tilde{\mathbb{1}} \mathcal{K}_{\Xi}\right)+\operatorname{tr} \log \left[\mathbb{1}-\mathcal{K}_{\Xi}^{-1}\left(-\not P-\mathbf{X}_{\Xi \Xi}+\mathbf{X}_{\Xi \xi} \boldsymbol{\Delta}_{\xi}^{-1}(q) \tilde{\mathbf{X}}_{\xi \Xi}\right)\right]
\end{aligned}
$$

where the first term on the r.h.s. of (2.68) is absorbed in the normalization of the path integral. Inserting $\boldsymbol{\Delta}_{\xi}^{-1}(q)$ from (2.55) yields

$$
\mathcal{L}_{\mathrm{EFT}, \mathrm{F}}^{1 \ell}=\frac{i}{2} \sum_{n=1}^{\infty} \frac{1}{n} \operatorname{tr}\left[\mathcal{K}_{\Xi}^{-1}\left(-\not P-\mathbf{X}_{\Xi \Xi}+\mathbf{X}_{\Xi \xi} \sum_{m=0}^{\infty}\left[\mathcal{K}_{\xi}^{-1} \mathbf{X}_{\xi \xi}\right]^{m} \mathcal{K}_{\xi}^{-1} \mathbf{X}_{\xi \Xi}\right)\right]^{n} .
$$

In order to obtain the final UOLEA from the sum

$$
\mathcal{L}_{\mathrm{EFT}}^{1 \ell}=\mathcal{L}_{\mathrm{EFT}, \mathrm{SF}}^{1 \ell}+\mathcal{L}_{\mathrm{EFT}, \mathrm{F}}^{1 \ell}
$$

one needs to expand all functional traces on the r.h.s. of (2.70) to a given mass dimension and calculate the coefficients and operator structures. In this expansion we keep $P^{\mu}$ as a whole to obtain a manifestly gauge-invariant effective Lagrangian. It can be shown, by using the Baker-Campbell-Hausdorff formula, that every $P_{\mu}$ appears in commutators of the form $\left[P_{\mu}, \bullet\right][11,12]$. To combine all $P^{\mu}$ operators into commutators one can either explicitly use the Baker-Campbell-Hausdorff formula in the calculation as was done in [8] or construct a basis for these commutators and then solve a system of equations to fix the coefficients of the basis elements as was pointed out in [18]. In this publication the second method was deployed. Our final expression for $\mathcal{L}_{\mathrm{EFT}}^{1 \ell}$ is contained in the file UOLEA.m of the arXiv submission and will be described further in the next section.

\section{Discussion of the result}

\subsection{Published operators and coefficients}

In the following we describe the calculated scalar/fermionic operators, which we publish in the file UOLEA .m of the arXiv submission. The file contains the following four lists:

- mixedLoopsNoP: mixed scalar/fermionic operators without $P^{\mu}$.

- mixedLoopsWithP: mixed scalar/fermionic operators with $P^{\mu}$.

- fermionicLoopsNoP: purely fermionic operators without $P^{\mu}$.

- fermionicLoopsWithP: purely fermionic operators with $P^{\mu}$.

For convenience, the additional list uolea is defined, which is the union of the four lists from above. The lists contain the calculated operators in the form $\left\{F^{\alpha}\left(M_{i}, M_{j}, \ldots\right), \mathcal{O}_{i j \ldots}^{\alpha}\right\}$, where $F^{\alpha}\left(M_{i}, M_{j}, \ldots\right)$ is the coefficient of the operator $\mathcal{O}_{i j \ldots}^{\alpha}$, which is expressed through 
the integrals $\tilde{\mathcal{I}}\left[q^{2 n_{c}}\right]_{i j \ldots 0}^{n_{i} n_{j} \ldots n_{L}}$ defined in appendix B. The operators $\mathcal{O}_{i j \ldots}^{\alpha}$ are expressed in terms of the symbols $X[\mathrm{~A}, \mathrm{~B}][i, j]$, with $\mathrm{A}, \mathrm{B} \in\{\mathrm{S}, \mathrm{s}, \mathrm{F}, \mathrm{f}\}$, which correspond to the matrices defined in section 2.2 as follows:

$$
\begin{aligned}
& X[\mathrm{~S}, \mathrm{~F}] \equiv \mathbf{X}_{\Phi \Xi}=\left(\begin{array}{ccc}
X_{\Sigma^{*} \Omega} & X_{\Sigma^{*} \bar{\Omega}} \mathcal{C}^{-1} & X_{\Sigma^{*} \Lambda} \\
X_{\Sigma \Omega} & X_{\Sigma \bar{\Omega}} \mathcal{C}^{-1} & X_{\Sigma \Lambda} \\
X_{\Theta \Omega} & X_{\Theta \bar{\Omega}} \mathcal{C}^{-1} & X_{\Theta \Lambda}
\end{array}\right), \\
& X[\mathrm{~s}, \mathrm{~F}] \equiv \mathbf{X}_{\phi \Xi}=\left(\begin{array}{ccc}
X_{\sigma^{*} \Omega} & X_{\sigma^{*} \bar{\Omega}} \mathcal{C}^{-1} & X_{\sigma^{*} \Lambda} \\
X_{\sigma \Omega} & X_{\sigma \bar{\Omega}} \mathcal{C}^{-1} & X_{\sigma \Lambda} \\
X_{\theta \Omega} & X_{\theta \bar{\Omega}} \mathcal{C}^{-1} & X_{\theta \Lambda}
\end{array}\right) \\
& X[\mathrm{~S}, \mathrm{f}] \equiv \mathbf{X}_{\Phi \xi}=\left(\begin{array}{ccc}
X_{\Sigma^{*} \omega} & X_{\Sigma^{*} \bar{\omega}} \mathcal{C}^{-1} & X_{\Sigma^{*} \lambda} \\
X_{\Sigma \omega} & X_{\Sigma \bar{\omega}} \mathcal{C}^{-1} & X_{\Sigma \lambda} \\
X_{\Theta \omega} & X_{\Theta \bar{\omega}} \mathcal{C}^{-1} & X_{\Theta \lambda}
\end{array}\right) \\
& X[\mathrm{~s}, \mathrm{f}] \equiv \mathbf{X}_{\phi \xi}=\left(\begin{array}{ccc}
X_{\sigma^{*} \omega} & X_{\sigma^{*} \bar{\omega}} \mathcal{C}^{-1} & X_{\sigma^{*} \lambda} \\
X_{\sigma \omega} & X_{\sigma \bar{\omega}} \mathcal{C}^{-1} & X_{\sigma \lambda} \\
X_{\theta \omega} & X_{\theta \bar{\omega}} \mathcal{C}^{-1} & X_{\theta \lambda}
\end{array}\right) \\
& X[\mathrm{~F}, \mathrm{~S}] \equiv \mathbf{X}_{\Xi \Phi}=\left(\begin{array}{ccc}
X_{\bar{\Omega} \Sigma} & X_{\bar{\Omega} \Sigma^{*}} & X_{\bar{\Omega} \Theta} \\
\mathcal{C}^{-1} X_{\Omega \Sigma} & \mathcal{C}^{-1} X_{\Omega \Sigma^{*}} & \mathcal{C}^{-1} X_{\Omega \Theta} \\
\mathcal{C}^{-1} X_{\Lambda \Sigma} & \mathcal{C}^{-1} X_{\Lambda \Sigma^{*}} & \mathcal{C}^{-1} X_{\Lambda \Theta}
\end{array}\right) \text {, } \\
& X[\mathrm{f}, \mathrm{S}] \equiv \mathbf{X}_{\xi \Phi}=\left(\begin{array}{ccc}
X_{\bar{\omega} \Sigma} & X_{\bar{\omega} \Sigma^{*}} & X_{\bar{\omega} \Theta} \\
\mathcal{C}^{-1} X_{\omega \Sigma} & \mathcal{C}^{-1} X_{\omega \Sigma^{*}} & \mathcal{C}^{-1} X_{\omega \Theta} \\
\mathcal{C}^{-1} X_{\lambda \Sigma} & \mathcal{C}^{-1} X_{\lambda \Sigma^{*}} & \mathcal{C}^{-1} X_{\lambda \Theta}
\end{array}\right), \\
& X[\mathrm{~F}, \mathrm{~s}] \equiv \mathbf{X}_{\Xi \phi}=\left(\begin{array}{ccc}
X_{\bar{\Omega} \sigma} & X_{\bar{\Omega} \sigma^{*}} & X_{\bar{\Omega} \theta} \\
\mathcal{C}^{-1} X_{\Omega \sigma} & \mathcal{C}^{-1} X_{\Omega \sigma^{*}} & \mathcal{C}^{-1} X_{\Omega \theta} \\
\mathcal{C}^{-1} X_{\Lambda \sigma} & \mathcal{C}^{-1} X_{\Lambda \sigma^{*}} & \mathcal{C}^{-1} X_{\Lambda \theta}
\end{array}\right), \\
& X[\mathrm{f}, \mathrm{s}] \equiv \mathbf{X}_{\xi \phi}=\left(\begin{array}{ccc}
X_{\bar{\omega} \sigma} & X_{\bar{\omega} \sigma^{*}} & X_{\bar{\omega} \theta} \\
\mathcal{C}^{-1} X_{\omega \sigma} & \mathcal{C}^{-1} X_{\omega \sigma^{*}} & \mathcal{C}^{-1} X_{\omega \theta} \\
\mathcal{C}^{-1} X_{\lambda \sigma} & \mathcal{C}^{-1} X_{\lambda \sigma^{*}} & \mathcal{C}^{-1} X_{\lambda \theta}
\end{array}\right), \\
& X[\mathrm{~F}, \mathrm{~F}] \equiv \mathbf{X}_{\Xi \Xi}=\left(\begin{array}{ccc}
X_{\bar{\Omega} \Omega} & X_{\bar{\Omega} \bar{\Omega}} \mathcal{C}^{-1} & X_{\bar{\Omega} \Lambda} \\
\mathcal{C}^{-1} X_{\Omega \Omega} & \mathcal{C}^{-1} X_{\Omega \bar{\Omega}} \mathcal{C}^{-1} & \mathcal{C}^{-1} X_{\Omega \Lambda} \\
\mathcal{C}^{-1} X_{\Lambda \Omega} & \mathcal{C}^{-1} X_{\Lambda \bar{\Omega}} \mathcal{C}^{-1} & \mathcal{C}^{-1} X_{\Lambda \Lambda}
\end{array}\right) \text {, } \\
& X[\mathrm{f}, \mathrm{f}] \equiv \mathbf{X}_{\xi \xi}=\left(\begin{array}{ccc}
X_{\bar{\omega} \omega} & X_{\bar{\omega} \bar{\omega}} \mathcal{C}^{-1} & X_{\bar{\omega} \lambda} \\
\mathcal{C}^{-1} X_{\omega \omega} & \mathcal{C}^{-1} X_{\omega \bar{\omega}} \mathcal{C}^{-1} & \mathcal{C}^{-1} X_{\omega \lambda} \\
\mathcal{C}^{-1} X_{\lambda \omega} & \mathcal{C}^{-1} X_{\lambda \bar{\omega}} \mathcal{C}^{-1} & \mathcal{C}^{-1} X_{\lambda \lambda}
\end{array}\right) \\
& X[\mathrm{~F}, \mathrm{f}] \equiv \mathbf{X}_{\Xi \xi}=\left(\begin{array}{ccc}
X_{\bar{\Omega} \omega} & X_{\bar{\Omega} \bar{\omega}} \mathcal{C}^{-1} & X_{\bar{\Omega} \lambda} \\
\mathcal{C}^{-1} X_{\Omega \omega} & \mathcal{C}^{-1} X_{\Omega \bar{\omega}} \mathcal{C}^{-1} & \mathcal{C}^{-1} X_{\Omega \lambda} \\
\mathcal{C}^{-1} X_{\Lambda \omega} & \mathcal{C}^{-1} X_{\Lambda \bar{\omega}} \mathcal{C}^{-1} & \mathcal{C}^{-1} X_{\Lambda \lambda}
\end{array}\right), \\
& X[\mathrm{f}, \mathrm{F}] \equiv \mathbf{X}_{\xi \Xi}=\left(\begin{array}{ccc}
X_{\bar{\omega} \Omega} & X_{\bar{\omega} \bar{\Omega}} \mathcal{C}^{-1} & X_{\bar{\omega} \Lambda} \\
\mathcal{C}^{-1} X_{\omega \Omega} & \mathcal{C}^{-1} X_{\omega \bar{\Omega}} \mathcal{C}^{-1} & \mathcal{C}^{-1} X_{\omega \Lambda} \\
\mathcal{C}^{-1} X_{\lambda \Omega} & \mathcal{C}^{-1} X_{\lambda \bar{\Omega}} \mathcal{C}^{-1} & \mathcal{C}^{-1} X_{\lambda \Lambda}
\end{array}\right)
\end{aligned}
$$




$$
\begin{aligned}
X[\mathrm{~S}, \mathrm{~S}] \equiv \mathbf{X}_{\Phi \Phi} & =\left(\begin{array}{ccc}
X_{\Sigma^{*} \Sigma} & X_{\Sigma^{*} \Sigma^{*}} & X_{\Sigma^{*} \Theta} \\
X_{\Sigma \Sigma} & X_{\Sigma \Sigma^{*}} & X_{\Sigma \Theta} \\
X_{\Theta \Sigma} & X_{\Theta \Sigma^{*}} & X_{\Theta \Theta}
\end{array}\right), \\
X[\mathrm{~S}, \mathrm{~s}] \equiv \mathbf{X}_{\Phi \phi} & =\left(\begin{array}{ccc}
X_{\Sigma^{*} \sigma} & X_{\Sigma^{*} \sigma^{*}} & X_{\Sigma^{*} \theta} \\
X_{\Sigma \sigma} & X_{\Sigma \sigma^{*}} & X_{\Sigma \theta} \\
X_{\Theta \sigma} & X_{\Theta \sigma^{*}} & X_{\Theta \theta}
\end{array}\right), \\
X[\mathrm{~s}, \mathrm{~S}] \equiv \mathbf{X}_{\phi \Phi} & =\left(\begin{array}{ccc}
X_{\sigma^{*} \Sigma} & X_{\sigma^{*} \Sigma^{*}} & X_{\sigma^{*} \Theta} \\
X_{\sigma \Sigma} & X_{\sigma \Sigma^{*}} & X_{\sigma \Theta} \\
X_{\theta \Sigma} & X_{\theta \Sigma^{*}} & X_{\theta \Theta}
\end{array}\right), \\
X[\mathrm{~s}, \mathrm{~s}] \equiv \mathbf{X}_{\phi \phi} & =\left(\begin{array}{ccc}
X_{\sigma^{*} \sigma} & X_{\sigma^{*} \sigma^{*}} & X_{\sigma^{*} \theta} \\
X_{\sigma \sigma} & X_{\sigma \sigma^{*}} & X_{\sigma \theta} \\
X_{\theta \sigma} & X_{\theta \sigma^{*}} & X_{\theta \theta}
\end{array}\right) .
\end{aligned}
$$

The indices $i, j \in \mathbb{N}$ label a specific element of the respective matrix. The full one-loop effective action is then obtained as

$$
\mathcal{L}_{\mathrm{EFT}}^{1 \ell}=\kappa \sum_{\alpha} \sum_{i j \ldots} F^{\alpha}\left(M_{i}, M_{j}, \ldots\right) \mathcal{O}_{i j \ldots}^{\alpha},
$$

where $\kappa=1 /(4 \pi)^{2}$ and the sum over $\alpha$ runs over all operators and their corresponding coefficients. Several comments regarding the use of the operators of (3.1) are in order. First, no assumptions have been made about the dependence of the second derivatives $X_{A B}$ regarding gamma matrices. The result is valid for any spin $1 / 2$ spinor structure appearing in these derivatives. Second, care has to be taken to retain the poles of the coefficients since the gamma algebra has to be performed in $d=4-\epsilon$ dimensions, which may generate finite contributions when combined with the poles. The function ExpandEps, contained in the Mathematica file LoopFunctions.m of the arXiv submission, can be used to extract these finite contributions. Third, some of the coefficients diverge in the case of degenerate masses if the degenerate limit is not taken carefully. The most convenient way to deal with degenerate masses may be to first set the masses equal, which modifies the integrals appearing in the coefficients $F^{\alpha}\left(M_{i}, M_{j}, \ldots\right)$, and to then calculate these integrals using the reduction algorithm implemented in the Mathematica file LoopFunctions.m of the arXiv submission. Last, there are no $c_{s}$ or $c_{F}$ factors appearing in the final result, in contrast to [8-10]. In our formulation these prefactors have been fixed by our treatment of the different kinds of fields and are absorbed in the coefficients.

\subsection{Infrared and ultra-violet divergences}

It appears that the operator coefficients have infrared divergences, which might be surprising as the infrared physics should cancel in the matching. The reason for the appearance of such poles is the fact that expansion by regions was used to perform the calculation as discussed in section 2.1. For a heavy-light loop this means that the one-loop integral $I_{\text {full }}$ in the full integration region is split into a part $I_{\text {soft }}$, calculated in the soft region, and a part $I_{\text {hard }}$, calculated in the hard region,

$$
I_{\text {full }}=I_{\text {soft }}+I_{\text {hard }} .
$$


Only the hard part remains, since the soft part is canceled in the matching by the EFT contribution. For the example of $I_{\text {full }}$ being finite, a UV-divergence in the soft part of the integration region cancels with an IR-divergence in the hard part with the condition

$$
\frac{1}{\epsilon_{\mathrm{UV}}}=\frac{1}{\epsilon_{\mathrm{IR}}}
$$

which assures that scaleless integrals vanish in dimensional regularization. Since the soft part is removed in the matching, the IR-divergence of the hard part remains. However, such an IR-divergence should be interpreted as a subtracted UV-divergence coming from the EFT as indicated by (3.3). It is not surprising that these divergences do not cancel in the matching since the UV behavior of the EFT is modified as compared to the UV-theory. However, since these genuine UV-divergences may still combine with an $\epsilon$ from the gamma algebra to yield finite contributions they must be treated in the same way as $1 / \epsilon$ poles stemming from the UV behavior of the UV-theory. After performing the trace and the gamma algebra, remaining terms containing $1 / \epsilon$ poles can be discarded, which amounts to performing a matching calculation in the $\overline{\mathrm{MS}}$ scheme.

\subsection{Application to models with massive vector fields}

The operators calculated in this paper can be used to treat massive vector fields in Feynman gauge as described in [18]. Furthermore, couplings of fermions to massless gauge bosons can be correctly accounted for as well using the same technique and the treatment is complete when the UV-theory is renormalizable. This follows from the fact that the gauge-kinetic term of a fermion $\psi$ is linear in the covariant derivative so that $X_{A_{\mu} \psi}$ is independent of $P_{\mu}$. This is not the case for scalar fields, since the kinetic term is quadratic in $P_{\mu}$, which means that even for a renormalizable UV-theory there are further operators stemming from the coupling of scalar fields to massless gauge bosons. Of course, once one considers the matching of a UV-theory that already contains higher dimensional operators with covariant derivatives to an EFT, further operators arise also for fermions. These missing operators all stem from open covariant derivatives and are currently unknown.

\subsection{Extraction of $\beta$-functions}

As was pointed out in [15] functional methods can be used to calculate $\beta$-functions since they allow for the computation of the loop-corrected generator of 1PI Green's functions. To one-loop we have

$$
\Gamma[\Phi]=\Gamma^{\text {tree }}[\Phi]+\Gamma^{1 \ell}[\Phi]
$$

where $\Gamma^{\text {tree }}[\Phi]=S[\Phi]$ is the tree-level generator of 1PI Green's functions, which is simply the classical action. Assume that $\Gamma^{\text {tree }}[\Phi]$ contains a kinetic term $\mathcal{O}_{K}[\Phi]$ and an interaction term $g \mathcal{O}_{g}[\Phi]$. Then, in general, the one-loop contribution will contain corrections to these, which depend on the renormalization scale $\mu$, so that

$$
\Gamma[\Phi] \supset \int \mathrm{d}^{4} x\left\{a_{K}(\mu) \mathcal{O}_{K}[\Phi]+a_{g}(\mu) \mathcal{O}_{g}[\Phi]\right\} .
$$


Canonically normalizing the kinetic term for the field $\Phi$ yields

$$
\Gamma[\Phi] \supset \int \mathrm{d}^{4} x\left\{\mathcal{O}_{K}[\Phi]+a_{g}^{\prime}(\mu) \mathcal{O}_{g}[\Phi]\right\}
$$

where

$$
\mu \frac{\mathrm{d}}{\mathrm{d} \mu} a_{g}^{\prime}(\mu)=0
$$

due to the Callan-Symanzik equation [30, 31]. Eq. (3.7) can be solved for the one-loop $\beta$-function of the coupling $g$.

In a specific sense, the UOLEA represents an expression for $\Gamma^{1 \ell}$ of a model with operators up to dimension 6 , and it can thus be used to calculate the one-loop $\beta$-functions of all dimension 6 operators for any given Lagrangian as described above. In order to calculate $\Gamma^{1 \ell}$, the UOLEA operators (3.1) must be re-interpreted as follows: since one is interested in the full $\Gamma^{1 \ell}$, a distinction between heavy and light fields must not be made and all fields shall be treated as "heavy" fields. As a consequence, the one-loop effective action of a scalar theory is given by

$$
\Gamma[\Phi]=S[\Phi]+\frac{i}{2} \log \operatorname{det}\left(-\frac{\delta^{2} \mathcal{L}_{\text {int }}}{\delta \Phi \delta \Phi}\right),
$$

where $\Phi$ represents the collection of all scalar fields contained in the model. The expression on the r.h.s. of $(3.8)$ can be expanded as outlined e.g. in $[8,15,17]$ and one arrives at the heavy-only part of the UOLEA (3.1), which contains only operators built out of derivatives of the Lagrangian with respect to "heavy" $\Phi$ fields. This procedure is not restricted to a theory with only scalars and can also be applied to models with both scalars and fermions using the heavy-only part of (3.1). However, higher-dimensional operators with covariant derivatives have not been treated in this work and hence their influence on the running of the couplings cannot be determined using our result.

\section{Applications}

\subsection{Integrating out the top quark from the Standard Model}

As a simple first example we consider the corrections to the Higgs tadpole and mass parameter that arise when integrating out the top quark from the Standard Model. The considered interaction Lagrangian shall contain only one coupling

$$
\mathcal{L}_{\mathrm{SM}} \supset-\frac{g_{t}}{\sqrt{2}} h \bar{t} t
$$

where $h$ denotes the physical Higgs field, $t$ is the top quark and $g_{t}$ is the top Yukawa coupling. The relevant operators of the UOLEA (2.70) are given by

$$
\begin{aligned}
\frac{1}{\kappa} \mathcal{L}_{\mathrm{EFT}}^{1 \ell}=\operatorname{tr}\{ & \frac{1}{4} m_{\Xi i} m_{\Xi j}^{3} \tilde{\mathcal{I}}_{i j}^{13}\left[P_{\mu},\left(\mathbf{X}_{\Xi \Xi}\right)_{i j}\right]\left[P^{\mu},\left(\mathbf{X}_{\Xi \Xi}\right)_{j i}\right] \\
& -\frac{1}{2} \tilde{\mathcal{I}}\left[q^{4}\right]_{i j}^{22} \gamma^{\nu}\left[P_{\mu},\left(\mathbf{X}_{\Xi \Xi}\right)_{i j}\right] \gamma_{\nu}\left[P^{\mu},\left(\mathbf{X}_{\Xi \Xi}\right)_{j i}\right]
\end{aligned}
$$




$$
\begin{aligned}
& -\tilde{\mathcal{I}}\left[q^{4}\right]_{i j}^{22} \gamma^{\nu}\left[P_{\nu},\left(\mathbf{X}_{\Xi \Xi}\right)_{i j}\right] \gamma_{\mu}\left[P^{\mu},\left(\mathbf{X}_{\Xi \Xi}\right)_{j i}\right] \\
& +\frac{1}{2} m_{\Xi i} \tilde{\mathcal{I}}_{i}^{1}\left(\mathbf{X}_{\Xi \Xi}\right)_{i i} \\
& -\frac{1}{4} m_{\Xi i} m_{\Xi j} \tilde{\mathcal{I}}_{i j}^{11}\left(\mathbf{X}_{\Xi \Xi}\right)_{i j}\left(\mathbf{X}_{\Xi \Xi}\right)_{j i} \\
& \left.-\frac{1}{4} \tilde{\mathcal{I}}\left[q^{2}\right]_{i j}^{11} \gamma^{\mu}\left(\mathbf{X}_{\Xi \Xi}\right)_{i j} \gamma_{\mu}\left(\mathbf{X}_{\Xi \Xi}\right)_{j i}\right\}
\end{aligned}
$$

where $m_{\Xi i}$ denotes the mass of the $i$ th component of $\Xi$. The matrix $\left(\mathbf{X}_{\Xi \Xi}\right)$ is given by

$$
\left(\mathbf{X}_{\Xi \Xi}\right)_{\alpha \beta i j}=\left(\begin{array}{cc}
\left(X_{\bar{t} t}\right)_{\alpha \beta i j} & 0 \\
0 & \mathcal{C}_{\alpha \rho}^{-1}\left(X_{t \bar{t}}\right)_{\rho \sigma i j} \mathcal{C}_{\sigma \beta}^{-1}
\end{array}\right)=-\frac{g_{t}}{\sqrt{2}} h \delta_{\alpha \beta} \delta_{i j} \mathbf{1}_{2 \times 2},
$$

with $\alpha, \beta=1, \ldots, 4$ being spinor indices and $i, j=1,2,3$ being color indices. In (4.2) we included terms with two covariant derivatives in order to obtain the field-redefinition of the Higgs field that is necessary to canonically normalize the corresponding Higgs field $\hat{h}$ in the effective theory. Since this redefinition arises from the correction to the kinetic term only, we can set $P^{\mu}=i \partial^{\mu}$. Inserting (4.3) into (4.2) and calculating the trace yields

$$
\begin{aligned}
\frac{1}{\kappa} \mathcal{L}_{\mathrm{EFT}}^{1 \ell}= & -3 g_{t}^{2}\left(m_{t}^{4} \tilde{\mathcal{I}}_{t}^{4}-2 d \tilde{\mathcal{I}}\left[q^{4}\right]_{t}^{4}-4 \tilde{\mathcal{I}}\left[q^{4}\right]_{t}^{4}\right)\left(\partial_{\mu} h\right)\left(\partial^{\mu} h\right) \\
& -3 g_{t}^{2}\left(\tilde{\mathcal{I}}_{t}^{2} m_{t}^{2}+d \tilde{\mathcal{I}}\left[q^{2}\right]_{t}^{2}\right) h^{2}-\frac{12}{\sqrt{2}} g_{t} m_{t} \tilde{\mathcal{I}}_{t}^{1} h,
\end{aligned}
$$

where $d=4-\epsilon=g_{\mu}^{\mu}$ has to be retained since the integrals contain poles in $1 / \epsilon$. The loop functions $\tilde{\mathcal{I}}$ are defined in appendix B. It is customary to introduce the canonically normalized field $\hat{h}$ which is related to $h$ through

$$
\hat{h}=\left(1+\frac{1}{2} \delta Z_{h}\right) h
$$

From (4.4) one can read off $\delta Z_{h}$ to be

$$
\delta Z_{h}=-6 g_{t}^{2}\left(m_{t}^{4} \tilde{\mathcal{I}}_{t}^{4}-2 d \tilde{\mathcal{I}}\left[q^{4}\right]_{t}^{4}-4 \tilde{\mathcal{I}}\left[q^{4}\right]_{t}^{4}\right)=-6 g_{t}^{2}\left(m_{t}^{4} \tilde{\mathcal{I}}_{t}^{4}-12 \tilde{\mathcal{I}}\left[q^{4}\right]_{t}^{4}+\frac{1}{6}\right)
$$

The loop functions that appear in (4.4) and (4.6) can be calculated with the Mathematica file LoopFunctions.m and read

$$
\begin{aligned}
& \tilde{\mathcal{I}}_{t}^{1}=2 \tilde{\mathcal{I}}\left[q^{2}\right]_{t}^{2}=m_{t}^{2}\left(\frac{2}{\epsilon}+1-\log \frac{m_{t}^{2}}{\mu^{2}}\right), \\
& \tilde{\mathcal{I}}_{t}^{2}=24 \tilde{\mathcal{I}}\left[q^{4}\right]_{t}^{4}=\frac{2}{\epsilon}-\log \frac{m_{t}^{2}}{\mu^{2}}, \\
& \tilde{\mathcal{I}}_{t}^{4}=\frac{1}{6 m_{t}^{4}} .
\end{aligned}
$$

\subsection{MSSM threshold correction to the quartic Higgs coupling}

As a first nontrivial application and a check we reproduce the one-loop threshold correction of the quartic Higgs coupling $\lambda$ when matching the MSSM to the SM at one-loop [32] in 
the unbroken phase. As discussed in [32] there are several contributions of distinct origins. The scalar contribution $\Delta \lambda^{1 \ell, \phi}$ arises from interactions of the SM-like Higgs with heavy Higgs bosons, squarks and sleptons, and the relevant interaction Lagrangian is given by

$$
\begin{aligned}
\mathcal{L}_{\phi}= & -\frac{g_{t}^{2}}{2} h^{2}\left(\tilde{t}_{L}^{*} \tilde{t}_{L}+\tilde{t}_{R}^{*} \tilde{t}_{R}\right)-\frac{1}{\sqrt{2}} g_{t} X_{t} h\left(\tilde{t}_{L}^{*} \tilde{t}_{R}+\tilde{t}_{L} \tilde{t}_{R}^{*}\right) \\
& -\frac{1}{8} c_{2 \beta} h^{2} \sum_{i}\left[\left(g_{2}^{2}-\frac{g_{1}^{2}}{5}\right) \tilde{u}_{L i}^{*} \tilde{u}_{L i}+\frac{4}{5} g_{1}^{2} \tilde{u}_{R i}^{*} \tilde{u}_{R i}-\left(g_{2}^{2}+\frac{g_{1}^{2}}{5}\right) \tilde{d}_{L i}^{*} \tilde{d}_{L i}-\frac{2}{5} g_{1}^{2} \tilde{d}_{R i}^{*} \tilde{d}_{R i}\right] \\
& -\frac{1}{8} c_{2 \beta} h^{2} \sum_{i}\left[\left(g_{2}^{2}+g_{1}^{2} \frac{3}{5}\right) \tilde{\nu}_{L i}^{*} \tilde{\nu}_{L i}-\left(g_{2}^{2}-g_{1}^{2} \frac{3}{5}\right) \tilde{e}_{L i}^{*} \tilde{e}_{L i}-\frac{6}{5} g_{1}^{2} \tilde{e}_{R i}^{*} \tilde{e}_{R i}\right] \\
& +\frac{1}{16} c_{2 \beta}^{2}\left(\frac{3}{5} g_{1}^{2}+g_{2}^{2}\right) h^{2} A^{2}-\frac{1}{8}\left(\left(1+s_{2 \beta}^{2}\right) g_{2}^{2}-\frac{3}{5} g_{1}^{2} c_{2 \beta}^{2}\right) h^{2} H^{-} H^{+} \\
& -\frac{1}{16}\left(\frac{3}{5} g_{1}^{2}+g_{2}^{2}\right)\left(3 s_{2 \beta}^{2}-1\right) h^{2} H^{2}-\frac{1}{8}\left(\frac{3}{5} g_{1}^{2}+g_{2}^{2}\right) s_{2 \beta} c_{2 \beta} h^{3} H \\
& +\frac{1}{8}\left(\frac{3}{5} g_{1}^{2}+g_{2}^{2}\right) s_{2 \beta} c_{2 \beta} h^{2}\left(G^{-} H^{+}+H^{-} G^{+}\right)+\frac{1}{8}\left(\frac{3}{5} g_{1}^{2}+g_{2}^{2}\right) s_{2 \beta} c_{2 \beta} h^{2} G^{0} A .
\end{aligned}
$$

Here $g_{1}$ and $g_{2}$ are the GUT-normalized electroweak gauge couplings, $X_{t}$ is the stop mixing parameter, and $g_{t}=y_{t} s_{\beta}$ with $y_{t}$ being the MSSM top Yukawa coupling and $s_{\beta}=\sin (\beta)$. The three generations of left- and right-handed squarks and sleptons are denoted as $\tilde{u}_{L i}$, $\tilde{u}_{R i}, \tilde{d}_{L i}, \tilde{d}_{R i}, \tilde{e}_{L i}, \tilde{e}_{R i}, \tilde{\nu}_{L i}(i=1,2,3)$, respectively, where $\tilde{t}_{L} \equiv \tilde{u}_{L 3}$ and $\tilde{t}_{R} \equiv \tilde{u}_{R 3}$ are the left- and right-handed stops. Furthermore we have defined $h=\sqrt{2} \Re \mathfrak{e}\left(\mathcal{H}^{0}\right)$, where $\mathcal{H}^{0}$ is the neutral component of the SM-like Higgs doublet $\mathcal{H}$ related to the Higgs doublets $H_{u}$ and $H_{d}$ through

$$
\mathcal{H}=-c_{\beta} \varepsilon H_{d}^{*}+s_{\beta} H_{u},
$$

where $\varepsilon$ is the antisymmetric tensor with $\varepsilon_{12}=1$ and $c_{\beta}=\cos (\beta), s_{2 \beta}=\sin (2 \beta)$ and $c_{2 \beta}=\cos (2 \beta)$. The fields $G^{0}$ and $G^{ \pm}$are Goldstone bosons arising from the same Higgs doublet. The heavy Higgs bosons $H, A$ and $H^{ \pm}$arise from the heavy doublet $\mathcal{A}$, which is related to the MSSM doublets through

$$
\mathcal{A}=s_{\beta} \varepsilon H_{d}^{*}+c_{\beta} H_{u} .
$$

Note, that since we work in the unbroken phase, $\beta$ should not be regarded as a ratio of vacuum expectation values, but as the fine-tuned mixing angle which rotates the two MSSM Higgs doublets $H_{u}$ and $H_{d}$ into $\mathcal{H}$ and $\mathcal{A}$ as given in (4.11)-(4.12) [32]. The fermionic contribution $\Delta \lambda^{1 \ell, \chi}$ to the threshold correction of $\lambda$ originates from interactions of the Higgs boson with charginos $\tilde{\chi}_{i}^{+}(i=1,2)$ and neutralinos $\tilde{\chi}_{i}^{0}(i=1, \ldots, 4)$ described by the interaction Lagrangian

$$
\begin{aligned}
\mathcal{L}_{\chi}= & -\frac{g_{2}}{\sqrt{2}} h c_{\beta}\left(\overline{\tilde{\chi}_{1}^{+}} P_{R} \tilde{\chi}_{2}^{+}+\overline{\tilde{\chi}_{2}^{+}} P_{L} \tilde{\chi}_{1}^{+}\right)-\frac{g_{2}}{\sqrt{2}} h s_{\beta}\left(\overline{\tilde{\chi}_{2}^{+}} P_{R} \tilde{\chi}_{1}^{+}+\overline{\tilde{\chi}_{1}^{+}} P_{L} \tilde{\chi}_{2}^{+}\right) \\
& +i \frac{g_{Y}}{2 \sqrt{2}}\left(c_{\beta}-s_{\beta}\right) h \overline{\tilde{\chi}_{1}^{0}} \gamma^{5} \tilde{\chi}_{3}^{0}-\frac{g_{Y}}{2 \sqrt{2}}\left(c_{\beta}+s_{\beta}\right) h \overline{\tilde{\chi}_{1}^{0}} \tilde{\chi}_{4}^{0} \\
& -i \frac{g_{2}}{2 \sqrt{2}}\left(c_{\beta}-s_{\beta}\right) h \overline{\tilde{\chi}_{2}^{0}} \gamma^{5} \tilde{\chi}_{3}^{0}+\frac{g_{2}}{2 \sqrt{2}}\left(c_{\beta}+s_{\beta}\right) h \overline{\tilde{\chi}_{2}^{0}} \tilde{\chi}_{4}^{0}
\end{aligned}
$$

where $\overline{\tilde{\chi}_{i}^{0}}=\left(\tilde{\chi}_{i}^{0}\right)^{T} \mathcal{C}$ and $g_{Y}=\sqrt{3 / 5} g_{1}$. 
To calculate the one-loop threshold correction for $\lambda$, the following contributions with purely scalar and purely fermionic operators from our generic UOLEA (2.70) are relevant,

$$
\begin{aligned}
& \frac{1}{\kappa} \mathcal{L}_{\mathrm{EFT}}^{1 \ell}=\operatorname{tr}\left\{\frac{1}{2} \tilde{\mathcal{I}}_{i}^{1}\left(\mathbf{X}_{\Phi \Phi}\right)_{i i}+\frac{1}{2} \tilde{\mathcal{I}}\left[q^{2}\right]_{i j}^{22}\left[P_{\mu},\left(\mathbf{X}_{\Phi \Phi}\right)_{i j}\right]\left[P^{\mu},\left(\mathbf{X}_{\Phi \Phi}\right)_{j i}\right]\right. \\
& +\frac{1}{4} \tilde{\mathcal{I}}_{i j}^{11}\left(\mathbf{X}_{\Phi \Phi}\right)_{i j}\left(\mathbf{X}_{\Phi \Phi}\right)_{j i}+\frac{1}{6} \tilde{\mathcal{I}}_{i j k}^{111}\left(\mathbf{X}_{\Phi \Phi}\right)_{i j}\left(\mathbf{X}_{\Phi \Phi}\right)_{j k}\left(\mathbf{X}_{\Phi \Phi}\right)_{k i} \\
& +\frac{1}{8} \tilde{\mathcal{I}}_{i j k l}^{1111}\left(\mathbf{X}_{\Phi \Phi}\right)_{i j}\left(\mathbf{X}_{\Phi \Phi}\right)_{j k}\left(\mathbf{X}_{\Phi \Phi}\right)_{k l}\left(\mathbf{X}_{\Phi \Phi}\right)_{l i}+\frac{1}{2} \tilde{\mathcal{I}}_{i}^{1}\left(\mathbf{X}_{\Phi \phi}\right)_{i j}\left(\mathbf{X}_{\phi \Phi}\right)_{j i} \\
& -\frac{1}{8} m_{\Xi i} m_{\Xi j} m_{\Xi k} m_{\Xi l} \tilde{\mathcal{I}}_{i j k l}^{1111}\left(\mathbf{X}_{\Xi \Xi}\right)_{i j}\left(\mathbf{X}_{\Xi \Xi}\right)_{j k}\left(\mathbf{X}_{\Xi \Xi}\right)_{k l}\left(\mathbf{X}_{\Xi \Xi}\right)_{l i} \\
& -\frac{1}{2} m_{\Xi i} m_{\Xi j} \tilde{\mathcal{I}}\left[q^{2}\right]_{i j k l}^{1111}\left(\mathbf{X}_{\Xi \Xi}\right)_{i j}\left(\mathbf{X}_{\Xi \Xi}\right)_{j k} \gamma^{\mu}\left(\mathbf{X}_{\Xi \Xi}\right)_{k l} \gamma_{\mu}\left(\mathbf{X}_{\Xi \Xi}\right)_{l i} \\
& -\frac{1}{4} m_{\Xi i} m_{\Xi k} \tilde{\mathcal{I}}\left[q^{2}\right]_{i j k l}^{1111}\left(\mathbf{X}_{\Xi \Xi}\right)_{i j} \gamma^{\mu}\left(\mathbf{X}_{\Xi \Xi}\right)_{j k}\left(\mathbf{X}_{\Xi \Xi}\right)_{k l} \gamma_{\mu}\left(\mathbf{X}_{\Xi \Xi}\right)_{l i} \\
& -\frac{1}{8} g_{\mu \nu \rho \sigma} \tilde{\mathcal{I}}\left[q^{4}\right]_{i j k l}^{1111} \gamma^{\mu}\left(\mathbf{X}_{\Xi \Xi}\right)_{i j} \gamma^{\nu}\left(\mathbf{X}_{\Xi \Xi}\right)_{j k} \gamma^{\rho}\left(\mathbf{X}_{\Xi \Xi}\right)_{k l} \gamma^{\sigma}\left(\mathbf{X}_{\Xi \Xi}\right)_{l i} \\
& +\frac{1}{4} m_{\Xi i} m_{\Xi j}^{3} \tilde{\mathcal{I}}_{i j}^{13}\left[P_{\mu},\left(\mathbf{X}_{\Xi \Xi}\right)_{i j}\right]\left[P^{\mu},\left(\mathbf{X}_{\Xi \Xi}\right)_{j i}\right] \\
& -\frac{1}{2} \tilde{\mathcal{I}}\left[q^{4}\right]_{i j}^{22} \gamma^{\nu}\left[P_{\mu},\left(\mathbf{X}_{\Xi \Xi}\right)_{i j}\right] \gamma_{\nu}\left[P^{\mu},\left(\mathbf{X}_{\Xi \Xi}\right)_{j i}\right] \\
& \left.-\tilde{\mathcal{I}}\left[q^{4}\right]_{i j}^{22} \gamma^{\nu}\left[P_{\nu},\left(\mathbf{X}_{\Xi \Xi}\right)_{i j}\right] \gamma_{\mu}\left[P^{\mu},\left(\mathbf{X}_{\Xi \Xi}\right)_{j i}\right]\right\}
\end{aligned}
$$

where $\kappa=1 /(4 \pi)^{2}$. The operators containing covariant derivatives can be removed by a field-strength renormalization of the Higgs field to canonically normalize the kinetic term. This field renormalization propagates into every Higgs coupling that has a non-vanishing tree-level contribution and hence also into the quartic coupling.

Next, we compute the $\mathbf{X}_{A B}$ matrices as the second derivatives of the Lagrangian with respect to the different kinds of fields. We start with

$$
\mathbf{X}_{\Phi \Phi}=\left(\begin{array}{ccc}
X_{\Sigma^{*} \Sigma} & X_{\Sigma^{*} \Sigma^{*}} & X_{\Sigma^{*} \Theta} \\
X_{\Sigma \Sigma} & X_{\Sigma \Sigma^{*}} & X_{\Sigma \Theta} \\
X_{\Theta \Sigma} & X_{\Theta \Sigma^{*}} & X_{\Theta \Theta}
\end{array}\right)
$$

and define

$$
\Sigma=\left(\begin{array}{lllllll}
\tilde{u}_{L i} & \tilde{u}_{R i} & \tilde{d}_{L i} & \tilde{d}_{R i} & \tilde{e}_{L i} & \tilde{e}_{R i} & \tilde{\nu}_{L i} H^{+}
\end{array}\right)^{T}, \quad \Theta=(A H)^{T},
$$

where $i=1,2,3$ denotes the generation index. The non-vanishing derivatives with respect to two heavy scalar fields read

$$
\begin{aligned}
& X_{\tilde{u}_{L i}^{*} \tilde{u}_{L j}}=X_{\tilde{u}_{L i} \tilde{u}_{L j}^{*}}=\frac{1}{8} c_{2 \beta} h^{2} \delta_{i j}\left(g_{2}^{2}-\frac{1}{5} g_{1}^{2}\right)+\delta_{3 i} \delta_{3 j} \frac{g_{t}^{2}}{2} h^{2}, \\
& X_{\tilde{u}_{R i}^{*} \tilde{u}_{R j}}=X_{\tilde{u}_{R i} \tilde{u}_{R j}^{*}}=\frac{1}{10} c_{2 \beta} h^{2} \delta_{i j} g_{1}^{2}+\delta_{3 i} \delta_{3 j} \frac{g_{t}^{2}}{2} h^{2}, \\
& X_{\tilde{d}_{L i}^{*} \tilde{d}_{L j}}=X_{\tilde{d}_{L i} \tilde{d}_{L j}^{*}}=-\frac{1}{8} c_{2 \beta} h^{2} \delta_{i j}\left(g_{2}^{2}+\frac{1}{5} g_{1}^{2}\right)
\end{aligned}
$$




$$
\begin{aligned}
X_{\tilde{d}_{R i}^{*} \tilde{d}_{R j}} & =X_{\tilde{d}_{R i} \tilde{d}_{R j}^{*}}=\frac{1}{20} c_{2 \beta} h^{2} \delta_{i j} g_{1}^{2}, \\
X_{\tilde{e}_{L i}^{*} \tilde{e}_{L j}} & =X_{\tilde{e}_{L i} \tilde{e}_{L j}^{*}}=\frac{1}{8} c_{2 \beta} h^{2} \delta_{i j}\left(g_{2}^{2}-\frac{3}{5} g_{1}^{2}\right), \\
X_{\tilde{e}_{R i}^{*} \tilde{e}_{R j}} & =X_{\tilde{e}_{R i} \tilde{e}_{R j}^{*}}=-\frac{1}{20} c_{2 \beta} h^{2} \delta_{i j} g_{1}^{2}, \\
X_{\tilde{\nu}_{L i}^{*} \tilde{\nu}_{L j}} & =X_{\tilde{\nu}_{L i} \tilde{\nu}_{L j}^{*}}=\frac{1}{8} c_{2 \beta} h^{2} \delta_{i j}\left(g_{2}^{2}+\frac{3}{5} g_{1}^{2}\right), \\
X_{H^{+} H^{-}} & =X_{H^{-} H^{+}}=\frac{1}{8} h^{2}\left[\left(1+s_{2 \beta}^{2}\right) g_{2}^{2}-\frac{3}{5} g_{1}^{2} c_{2 \beta}^{2}\right] \\
X_{A A} & =-\frac{1}{16} c_{2 \beta}^{2}\left(\frac{3}{5} g_{1}^{2}+g_{2}^{2}\right) h^{2}, \\
X_{H H} & =\frac{1}{16}\left(2 s_{2 \beta}^{2}-1\right)\left(\frac{3}{5} g_{1}^{2}+g_{2}^{2}\right) h^{2}, \\
X_{\tilde{u}_{L i}^{*} \tilde{u}_{R j}} & =X_{\tilde{u}_{L i} \tilde{u}_{R j}^{*}}=\frac{1}{\sqrt{2}} \delta_{3 i} \delta_{3 j} g_{t} X_{t} h .
\end{aligned}
$$

Given these derivatives we find that $\mathbf{X}_{\Phi \Phi}$ is block-diagonal with the blocks being

$$
\begin{aligned}
& X_{\Sigma^{*} \Sigma}=\left(\begin{array}{ccc}
X_{\tilde{u}_{L i}^{*} \tilde{u}_{L j}} & X_{\tilde{u}_{L i}^{*} \tilde{u}_{R j}} & \mathbf{0}_{1 \times 6} \\
X_{\tilde{u}_{R i}^{*}}^{*} \tilde{u}_{L j} & X_{\tilde{u}_{R i}^{*}}^{*} \tilde{u}_{R j} & \mathbf{0}_{1 \times 6} \\
\mathbf{0}_{6 \times 1} & \mathbf{0}_{6 \times 1} & X_{\Pi^{*} \Pi}
\end{array}\right), \\
& X_{\Pi *} \Pi= \operatorname{diag}\left(X_{\tilde{d}_{L i}^{*} \tilde{d}_{L j}}, X_{\tilde{d}_{R i}^{*}} \tilde{d}_{R j}, X_{\tilde{e}_{L i}^{*} \tilde{e}_{L j}}, X_{\tilde{e}_{R i}^{*} \tilde{e}_{R j}}, X_{\tilde{\nu}_{L i}^{*} \tilde{\nu}_{L j}}, X_{H^{+} H^{-}}\right), \\
& X_{\Sigma \Sigma^{*}}=\left(\begin{array}{ccc}
X_{\tilde{u}_{L i} \tilde{u}_{L j}^{*}} & X_{\tilde{u}_{L i} \tilde{u}_{R j}^{*}} & \mathbf{0}_{1 \times 6} \\
X_{\tilde{u}_{R i} \tilde{u}_{L j}^{*}} & X_{\tilde{u}_{R i} \tilde{u}_{R j}^{*}} & \mathbf{0}_{1 \times 6} \\
\mathbf{0}_{6 \times 1} & \mathbf{0}_{6 \times 1} & X_{\Pi \Pi^{*}}
\end{array}\right), \\
& X_{\Pi \Pi \Pi^{*}}=\operatorname{diag}\left(X_{\tilde{d}_{L i} \tilde{d}_{L j}^{*}}, X_{\tilde{d}_{R i} \tilde{d}_{R j}^{*}}, X_{\tilde{e}_{L i} \tilde{e}_{L j}^{*}}, X_{\tilde{e}_{R i} \tilde{e}_{R j}^{*}}, X_{\tilde{\nu}_{L i} \tilde{\nu}_{L j}^{*}}, X_{H^{-} H^{+}}\right), \\
& X_{\Theta \Theta}=\operatorname{diag}\left(X_{A A}, X_{H H}\right),
\end{aligned}
$$

where $\mathbf{0}_{m \times n}$ denotes the $m \times n$ matrix of only zeros. We next calculate $\mathbf{X}_{\phi \Phi}$ and $\mathbf{X}_{\Phi \phi}$, which contain derivatives with respect to one heavy and one light scalar field. We define the light scalar field multiplets as

$$
\sigma=\left(G^{+}\right), \quad \theta=\left(h G^{0}\right)^{T} .
$$

As discussed in section 2.1 the derivatives w.r.t. the fields are evaluated at the background field configurations, and the heavy background fields are expressed in terms of the light ones using a local operator expansion. ${ }^{3}$ This corresponds to an expansion in $\square / M^{2}$ for a heavy scalar field of mass $M$ and hence it leads to contributions suppressed by at least $1 / M^{2}$. Since we are not interested in these suppressed contributions here, we only consider derivatives of the Lagrangian which exclusively contain light background fields and set all other derivatives to zero. The non-vanishing derivatives are given by

$$
X_{H h}=X_{h H}=\frac{3}{8}\left(\frac{3}{5} g_{1}^{2}+g_{2}^{2}\right) s_{2 \beta} c_{2 \beta} h^{2},
$$

\footnotetext{
${ }^{3}$ An explicit example is given in section 4.4 in the treatment of dimension 5 operators.
} 


$$
\begin{gathered}
X_{A G^{0}}=X_{G^{0} A}=-\frac{1}{8}\left(\frac{3}{5} g_{1}^{2}+g_{2}^{2}\right) s_{2 \beta} c_{2 \beta} h^{2}, \\
X_{H^{+} G^{-}}=X_{H^{-} G^{+}}=-\frac{1}{8}\left(\frac{3}{5} g_{1}^{2}+g_{2}^{2}\right) s_{2 \beta} c_{2 \beta} h^{2} .
\end{gathered}
$$

We then find that $\mathbf{X}_{\Phi \phi}$ is block-diagonal with the blocks being

$$
\begin{aligned}
X_{\Sigma^{*} \sigma} & =\left(\begin{array}{c}
\mathbf{0}_{7 \times 1} \\
X_{H^{-} G^{+}}
\end{array}\right), \\
X_{\Sigma \sigma^{*}} & =\left(\begin{array}{c}
\mathbf{0}_{7 \times 1} \\
X_{H^{+} G^{-}}
\end{array}\right), \\
X_{\Theta \theta} & =\left(\begin{array}{cc}
0 & X_{A G^{0}} \\
X_{H h} & 0
\end{array}\right) .
\end{aligned}
$$

Similarly, $\mathbf{X}_{\phi \Phi}$ is block-diagonal with diagonal entries

$$
\begin{aligned}
X_{\sigma^{*} \Sigma} & =\left(\begin{array}{ll}
\mathbf{0}_{1 \times 7} & X_{G^{-} H^{+}}
\end{array}\right), \\
X_{\sigma \Sigma^{*}} & =\left(\begin{array}{ll}
\mathbf{0}_{1 \times 7} & X_{G^{+} H^{-}}
\end{array}\right), \\
X_{\theta \Theta} & =\left(\begin{array}{cc}
0 & X_{h H} \\
X_{G^{0} A} & 0
\end{array}\right) .
\end{aligned}
$$

Finally, we need the derivatives with respect to two heavy fermions to construct the matrix $\mathbf{X}_{\Xi \Xi}$. We define

$$
\Omega=\left(\begin{array}{ll}
\tilde{\chi}_{1}^{+} & \tilde{\chi}_{2}^{+}
\end{array}\right)^{T}, \quad \Lambda=\left(\begin{array}{llll}
\tilde{\chi}_{1}^{0} & \tilde{\chi}_{2}^{0} & \tilde{\chi}_{3}^{0} & \tilde{\chi}_{4}^{0}
\end{array}\right)^{T}
$$

and the matrix $\mathbf{X}_{\Xi \Xi}$ is again block-diagonal with the non-vanishing entries

$$
\begin{aligned}
X_{\bar{\Omega} \Omega} & =\mathcal{C}^{-1} X_{\Omega \bar{\Omega}}^{T} \mathcal{C}^{-1}=-\frac{g_{2}}{\sqrt{2}} h\left(\begin{array}{ccc}
0 & c_{\beta} P_{R}+s_{\beta} P_{L} \\
c_{\beta} P_{L}+s_{\beta} P_{R} & 0
\end{array}\right), \\
\mathcal{C}^{-1} X_{\Lambda \Lambda} & =\frac{h}{2 \sqrt{2}}\left(\begin{array}{cccc}
0 & i g_{Y}\left(c_{\beta}-s_{\beta}\right) \gamma^{5} & -g_{Y}\left(c_{\beta}+s_{\beta}\right) \\
0 & 0 & -i g_{2}\left(c_{\beta}-s_{\beta}\right) \gamma^{5} & g_{2}\left(c_{\beta}+s_{\beta}\right) \\
i g_{Y}\left(c_{\beta}-s_{\beta}\right) \gamma^{5} & -i g_{2}\left(c_{\beta}-s_{\beta}\right) \gamma^{5} & 0 & 0 \\
-g_{Y}\left(c_{\beta}+s_{\beta}\right) & g_{2}\left(c_{\beta}+s_{\beta}\right) & 0 & 0
\end{array}\right),
\end{aligned}
$$

where the relations of appendix $\mathrm{C}$ were used to simplify the expressions. Note, that in the calculation of $X_{\Lambda \Lambda}$ for a given Majorana fermion $\lambda$ the two fields $\bar{\lambda}$ and $\lambda$ are not independent, but are related via $\bar{\lambda}=\lambda^{T} \mathcal{C}$. Inserting all of the derivatives into (4.14), summing over all indices and canonically normalizing the kinetic term for the SM-like Higgs boson as

$$
\begin{aligned}
h= & \left(1-\frac{1}{2} \delta Z_{h}\right) \hat{h}, \\
\delta Z_{h}= & -6 g_{t}^{2} X_{t}^{2} \tilde{\mathcal{I}}\left[q^{2}\right]_{\tilde{q} \tilde{u}}^{22}+\frac{s_{2 \beta}}{2} \mu\left(g_{Y}^{2} M_{1} \mu^{2} \tilde{\mathcal{I}}_{1 \mu}^{13}+g_{Y}^{2} M_{1}^{3} \tilde{\mathcal{I}}_{1 \mu}^{31}-3 g_{2}^{2} M_{2} \mu^{2} \tilde{\mathcal{I}}_{2 \mu}^{13}-3 g_{2}^{2} M_{2}^{3} \tilde{\mathcal{I}}_{2 \mu}^{31}\right) \\
& +2(2+d)\left(-g_{Y}^{2} \tilde{\mathcal{I}}\left[q^{4}\right]_{1 \mu}^{22}+3 g_{2}^{2} \tilde{\mathcal{I}}\left[q^{4}\right]_{2 \mu}^{22}\right),
\end{aligned}
$$


one finds the following effective Lagrangian

$$
\mathcal{L}_{\mathrm{EFT}}^{1 \ell}=\frac{1}{2}(\partial \hat{h})^{2}-\frac{\lambda}{8} \hat{h}^{4}+\cdots
$$

with

$$
\begin{aligned}
\lambda & =\frac{1}{4}\left(\frac{3}{5} g_{1}^{2}+g_{2}^{2}\right) c_{2 \beta}^{2}+\kappa \Delta \lambda^{1 \ell}, \\
\Delta \lambda^{1 \ell} & =\Delta \lambda^{1 \ell, \text { reg }}+\Delta \lambda^{1 \ell, \phi}+\Delta \lambda^{1 \ell, \chi},
\end{aligned}
$$

and

$$
\begin{aligned}
& \Delta \lambda^{1 \ell, \phi}=g_{t}^{4}\left[-3 X_{t}^{4} \tilde{\mathcal{I}}_{\tilde{q} \tilde{q} \tilde{u} \tilde{u}}^{1111}-6 X_{t}^{2}\left(\tilde{\mathcal{I}}_{\tilde{q} \tilde{q} \tilde{u}}^{111}+\tilde{\mathcal{I}}_{\tilde{q} \tilde{u} \tilde{u}}^{111}\right)-3\left(\tilde{\mathcal{I}}_{\tilde{q} \tilde{q}}^{11}+\tilde{\mathcal{I}}_{\tilde{u} \tilde{u}}^{11}\right)\right] \\
& +\frac{3}{10} g_{t}^{2} c_{2 \beta}\left\{X_{t}^{2}\left[2 c_{2 \beta}\left(3 g_{1}^{2}+5 g_{2}^{2}\right) \tilde{\mathcal{I}}\left[q^{2}\right]_{\tilde{q} \tilde{u}}^{22}+\left(g_{1}^{2}-5 g_{2}^{2}\right) \tilde{\mathcal{I}}_{\tilde{q} \tilde{q} \tilde{u}}^{111}-4 g_{1}^{2} \tilde{\mathcal{I}}_{\tilde{q} \tilde{u} \tilde{u}}^{111}\right]\right. \\
& \left.+\left(g_{1}^{2}-5 g_{2}^{2}\right) \tilde{\mathcal{I}}_{\tilde{q} \tilde{q}}^{11}-4 g_{1}^{2} \tilde{\mathcal{I}}_{\tilde{u} \tilde{u}}^{11}\right\} \\
& -\frac{c_{2 \beta}^{2}}{200} \sum_{i=1}^{3}\left[3\left(g_{1}^{4}+25 g_{2}^{4}\right) \tilde{\mathcal{I}}_{\tilde{q}_{i} \tilde{q}_{i}}^{11}+24 g_{1}^{4} \tilde{\mathcal{I}}_{\tilde{u}_{i} \tilde{u}_{i}}^{11}+6 g_{1}^{4} \tilde{\mathcal{I}}_{\tilde{d}_{i} \tilde{d}_{i}}^{11}\right. \\
& +\left(9 g_{1}^{4}+25 g_{2}^{4}\right) \tilde{\mathcal{I}}_{\tilde{l}_{i} \tilde{l}_{i}}^{11}+18 g_{1}^{4} \tilde{\mathcal{I}}_{\tilde{e}_{i} \tilde{e}_{i}}^{11} \\
& +\frac{1}{200}\left\{6 c_{2 \beta}^{2}\left(c_{2 \beta}^{2}-1\right)\left(3 g_{1}^{2}+5 g_{2}^{2}\right)^{2} \tilde{\mathcal{I}}_{A 0}^{11}-\left[9\left(3 c_{2 \beta}^{4}-3 c_{2 \beta}^{2}+1\right) g_{1}^{4}\right.\right. \\
& \left.\left.+30\left(3 c_{2 \beta}^{4}-4 c_{2 \beta}^{2}+1\right) g_{1}^{2} g_{2}^{2}+25\left(3 c_{2 \beta}^{4}-5 c_{2 \beta}^{2}+3\right) g_{2}^{4}\right] \tilde{\mathcal{I}}_{A A}^{11}\right\}, \\
& \Delta \lambda^{1 \ell, \chi}=-\frac{1}{4}\left\{-d\left(2 g_{Y}^{4} M_{1}^{2} \tilde{\mathcal{I}}\left[q^{2}\right]_{1 \mu}^{22}+2 g_{2}^{4} M_{2}^{2} \tilde{\mathcal{I}}\left[q^{2}\right]_{2 \mu}^{22}+g_{Y}^{4} \mu^{2} \tilde{\mathcal{I}}\left[q^{2}\right]_{1 \mu}^{22}\right.\right. \\
& -g_{Y}^{4} \mu^{2} c_{4 \beta} \tilde{\mathcal{I}}\left[q^{2}\right]_{1 \mu}^{22}+g_{2}^{4} \mu^{2} \tilde{\mathcal{I}}\left[q^{2}\right]_{2 \mu}^{22}-g_{2}^{4} \mu^{2} c_{4 \beta} \tilde{\mathcal{I}}\left[q^{2}\right]_{2 \mu}^{22} \\
& \left.+4 g_{Y}^{2} g_{2}^{2} M_{1} M_{2} \tilde{\mathcal{I}}\left[q^{2}\right]_{12 \mu}^{112}+2 g_{Y}^{2} g_{2}^{2} \mu^{2} \tilde{\mathcal{I}}\left[q^{2}\right]_{12 \mu}^{112}-2 g_{Y}^{2} g_{2}^{2} \mu^{2} c_{4 \beta} \tilde{\mathcal{I}}\left[q^{2}\right]_{12 \mu}^{112}\right) \\
& -d(2+d)\left(2 g_{Y}^{4} \tilde{\mathcal{I}}\left[q^{4}\right]_{1 \mu}^{22}+2 g_{2}^{4} \tilde{\mathcal{I}}\left[q^{4}\right]_{2 \mu}^{22}+4 g_{Y}^{2} g_{2}^{2} \tilde{\mathcal{I}}\left[q^{4}\right]_{12 \mu}^{112}\right) \\
& -g_{2}^{4}\left[2 d(2+d)\left(3+c_{4 \beta}\right) \tilde{\mathcal{I}}\left[q^{4}\right]_{2 \mu}^{22}+16 c_{\beta} s_{\beta}\left(d M_{2} \tilde{\mathcal{I}}\left[q^{2}\right]_{2 \mu}^{22}\left(\mu+M_{2} c_{\beta} s_{\beta}\right)\right.\right. \\
& \left.\left.+\mu\left\{M_{2}^{2} \mu c_{\beta} \tilde{\mathcal{I}}_{2 \mu}^{22} s_{\beta}+d \tilde{\mathcal{I}}\left[q^{2}\right]_{2 \mu}^{22}\left(M_{2}+\mu c_{\beta} s_{\beta}\right)\right\}\right)\right] \\
& -4 d \mu\left(2 g_{Y}^{4} M_{1} \tilde{\mathcal{I}}\left[q^{2}\right]_{1 \mu}^{22}+2 g_{2}^{4} M_{2} \tilde{\mathcal{I}}\left[q^{2}\right]_{2 \mu}^{22}+2 g_{Y}^{2} g_{2}^{2} M_{1} \tilde{\mathcal{I}}\left[q^{2}\right]_{12 \mu}^{112}\right. \\
& \left.+2 g_{Y}^{2} g_{2}^{2} M_{2} \tilde{\mathcal{I}}\left[q^{2}\right]_{12 \mu}^{112}\right) s_{2 \beta} \\
& -2 \mu^{2}\left(g_{Y}^{4} M_{1}^{2} \tilde{\mathcal{I}}_{1 \mu}^{22}+g_{2}^{2} M_{2}\left(g_{2}^{2} M_{2} \tilde{\mathcal{I}}_{2 \mu}^{22}+g_{Y}^{2} M_{1} 2 \tilde{\mathcal{I}}_{12 \mu}^{112}+\right)\right) s_{2 \beta}^{2} \\
& -2 g_{2}^{2}\left(g_{Y}^{2}+g_{2}^{2}\right) c_{2 \beta}^{2}\left(-4(2+d) \tilde{\mathcal{I}}\left[q^{4}\right]_{2 \mu}^{22}+M_{2} \mu\left(\mu^{2} \tilde{\mathcal{I}}_{2 \mu}^{13}+M_{2}^{2} \tilde{\mathcal{I}}_{2 \mu}^{31}\right) s_{2 \beta}\right) \\
& -\left(g_{Y}^{2}+g_{2}^{2}\right) c_{2 \beta}^{2}\left(-4(2+d) g_{Y}^{2} \tilde{\mathcal{I}}\left[q^{4}\right]_{1 \mu}^{22}-4(2+d) g_{2}^{2} \tilde{\mathcal{I}}\left[q^{4}\right]_{2 \mu}^{22}\right. \\
& \left.\left.+\mu\left\{g_{Y}^{2} M_{1} \mu^{2} \tilde{\mathcal{I}}_{1 \mu}^{13}+g_{Y}^{2} M_{1}^{3} \tilde{\mathcal{I}}_{1 \mu}^{31}+g_{2}^{2} M_{2}\left(\mu^{2} \tilde{\mathcal{I}}_{2 \mu}^{31}+M_{2}^{2} \tilde{\mathcal{I}}_{2 \mu}^{31}\right)\right\} s_{2 \beta}\right)\right\} .
\end{aligned}
$$


The subscripts 1 and 2 of the loop functions are shorthand for $M_{1}$ and $M_{2}$, respectively. The terms involving $d=4-\epsilon$ originate from contractions of gamma matrices and metric tensors, see appendix D. Note, that $\lambda$ is expressed entirely in terms of the MSSM gauge couplings, in contrast to [32].

It is sensible to regularize the MSSM using dimensional reduction (DRED) [33], whereas the SM is more naturally regularized in dimensional regularization (DREG) [3438]. Such a regularization scheme change leads to further contributions to the threshold correction denoted by $\Delta \lambda^{1 \ell \text {,reg }}$, which can be obtained using the DRED-DREG regularization scheme translating operators presented in [10]. This contribution originates from the operator

$$
\frac{1}{\kappa} \epsilon \mathcal{L}_{\mathrm{EFT}, \epsilon}^{1 \ell}=\frac{1}{2} \operatorname{tr}\left\{\breve{X}_{\epsilon \epsilon}^{\mu \nu} \breve{X}_{\epsilon \epsilon \mu \nu}\right\}
$$

where on the r.h.s. $\epsilon$ denotes all epsilon scalars that couple to the Higgs and

$$
\breve{X}_{\epsilon \epsilon}^{\mu \nu}=\breve{g}_{\sigma}^{\mu} \breve{g}_{\rho}^{\nu} \stackrel{\circ}{X}_{\epsilon \epsilon}^{\sigma \rho}
$$

is the projection of the 4-dimensional $\dot{X}_{\epsilon \epsilon}^{\sigma \rho}$ onto the $\epsilon$-dimensional $Q \epsilon S$ space $[10,39]$ with $\breve{g}^{\mu \nu} \breve{g}_{\mu \nu}=\epsilon$, see appendix D. In the MSSM we have the following couplings to epsilon scalars to the SM-like doublet $\mathcal{H}$,

$$
\mathcal{L}_{\epsilon \mathcal{H}}=\mathcal{H}_{i}^{*} \breve{g}_{\mu \nu}\left(g_{2}^{2} T_{i j}^{a} T_{j l}^{b} a^{a \mu} a^{b \nu}+\sqrt{\frac{3}{5}} g_{1} g_{2} T_{i l}^{a} a^{a \mu} b^{\nu}+\frac{3}{20} g_{1}^{2} b^{\mu} b^{\nu} \delta_{i l}\right) \mathcal{H}_{l},
$$

where the indices $i, j, l$ are $\mathrm{SU}(2)_{L}$ indices of the fundamental representation with the generators $T_{i j}^{a}$. The fields $a^{a \mu}$ and $b^{\mu}$ denote the epsilon scalars corresponding to $\mathrm{SU}(2)_{L}$ and $\mathrm{U}(1)_{Y}$, respectively. One obtains the derivative

$$
\breve{X}_{\epsilon \epsilon}^{\mu \nu}=-\breve{g}^{\mu \nu}\left(\begin{array}{cc}
\mathcal{H}_{i}^{*} g_{2}^{2}\left\{T^{a}, T^{b}\right\}_{i l} \mathcal{H}_{l} & \sqrt{\frac{3}{5}} g_{1} g_{2} \mathcal{H}_{i}^{*} T_{i l}^{a} \mathcal{H}_{l} \\
\sqrt{\frac{3}{5}} g_{1} g_{2} \mathcal{H}_{i}^{*} T_{i l}^{a} \mathcal{H}_{l} & \frac{3}{10} g_{1}^{2} \mathcal{H}_{i}^{*} \mathcal{H}_{i}
\end{array}\right) .
$$

Inserting this into (4.53) we obtain

$$
\Delta \lambda^{1 \ell, \text { reg }}=-\frac{9}{100} g_{1}^{4}-\frac{3}{10} g_{1}^{2} g_{2}^{2}-\frac{3}{4} g_{2}^{4} .
$$

We do not find the term proportional to $c_{2 \beta}^{2}$ given in [32] since this term only arises once the tree-level expression for $\lambda$ is expressed in terms of SM gauge couplings, as opposed to MSSM parameters as in our case. Up to terms arising from this conversion the one-loop threshold corrections agree with the results of [32].

\subsection{Integrating out stops and the gluino from the MSSM}

As a second nontrivial application we reproduce known threshold corrections from the MSSM to the Standard Model Effective Field Theory (SMEFT) from heavy stops and the gluino in the gaugeless limit $\left(g_{1}=g_{2}=0\right)$ in the unbroken phase and for vanishing Yukawa couplings, except for the one of the top quark. In particular we reproduce the 
Wilson coefficient of the higher-dimensional $\hat{h}^{6}$ operator calculated in $[8,40]$. Furthermore, this example application again represents a scenario, where a heavy Majorana fermion is integrated out and the formalism introduced in section 2 must be carefully applied.

We consider the following part of the MSSM Lagrangian

$$
\begin{aligned}
& \mathcal{L}_{\mathrm{MSSM}} \supset\left|\partial \tilde{t}_{L}\right|^{2}-m_{\tilde{q}}^{2}\left|\tilde{t}_{L}\right|^{2}+\left|\partial \tilde{t}_{R}\right|^{2}-m_{\tilde{u}}^{2}\left|\tilde{t}_{R}\right|^{2}+\frac{1}{2}\left(\tilde{g}^{a}\right)^{T} \mathcal{C}\left(i \not \partial-m_{\tilde{g}}\right) \tilde{g}^{a} \\
& -\frac{y_{t} s_{\beta}}{\sqrt{2}} h \bar{t} t-\frac{y_{t}^{2} s_{\beta}^{2}}{2} h^{2}\left(\left|\tilde{t}_{L}\right|^{2}+\left|\tilde{t}_{R}\right|^{2}\right)-\frac{y_{t} s_{\beta} X_{t}}{\sqrt{2}} h\left(\tilde{t}_{L}^{*} \tilde{t}_{R}+\text { h.c. }\right) \\
& -\sqrt{2} g_{3}\left[\bar{t} P_{R} \tilde{g}^{a} T^{a} \tilde{t}_{L}-\bar{t} P_{L} \tilde{g}^{a} T^{a} \tilde{t}_{R}+\tilde{t}_{L}^{*}\left(\tilde{g}^{a}\right)^{T} T^{a} \mathcal{C} P_{L} t-\tilde{t}_{R}^{*}\left(\tilde{g}^{a}\right)^{T} T^{a} \mathcal{C} P_{R} t\right],
\end{aligned}
$$

where we use the same notation as in section 4.2 and $g_{3}$ is the strong gauge coupling. The top quark is denoted as $t$ and is defined as a Dirac fermion built from the upper component of the left-handed quark-doublet $q_{L}$ and the right-handed top $t_{R}$. The gluino is denoted as $\tilde{g}^{a}$ and we have used the relation $\overline{\tilde{g}}^{a}=\left(\left(\tilde{g}^{a}\right)^{C}\right)^{T} \mathcal{C}=\left(\tilde{g}^{a}\right)^{T} \mathcal{C}$ to express (4.58) in terms of the gluino Majorana spinor $\tilde{g}^{a}$.

Upon integrating out the heavy stops and the gluino the Lagrangian of the effective theory becomes

$$
\mathcal{L}_{\mathrm{SMEFT}} \supset-\frac{y_{t} s_{\beta}}{\sqrt{2}} h \bar{t} t+\mathcal{L}_{\mathrm{SMEFT}}^{1 \ell}
$$

In our limit the one-loop term $\mathcal{L}_{\text {SMEFT }}^{1 \ell}$ receives contributions from the following generic operators from $(2.70)$

$$
\begin{aligned}
\frac{1}{\kappa} \mathcal{L}_{\mathrm{EFT}}^{1 \ell} \supset & \frac{1}{2} \tilde{\mathcal{I}}_{i}^{1}\left(\mathbf{X}_{\Phi \Phi}\right)_{i i}+\frac{1}{4} \tilde{\mathcal{I}}_{i k}^{11}\left(\mathbf{X}_{\Phi \Phi}\right)_{i k}\left(\mathbf{X}_{\Phi \Phi}\right)_{k i}+\frac{1}{6} \tilde{\mathcal{I}}_{l i k}^{111}\left(\mathbf{X}_{\Phi \Phi}\right)_{i k}\left(\mathbf{X}_{\Phi \Phi}\right)_{k l}\left(\mathbf{X}_{\Phi \Phi}\right)_{l i} \\
& +\frac{1}{8} \tilde{\mathcal{I}}_{l i k n}^{1111}\left(\mathbf{X}_{\Phi \Phi}\right)_{i k}\left(\mathbf{X}_{\Phi \Phi}\right)_{k l}\left(\mathbf{X}_{\Phi \Phi}\right)_{l n}\left(\mathbf{X}_{\Phi \Phi}\right)_{n i} \\
& +\frac{1}{10} \tilde{\mathcal{I}}_{i k l n p}^{11111}\left(\mathbf{X}_{\Phi \Phi}\right)_{i k}\left(\mathbf{X}_{\Phi \Phi}\right)_{k l}\left(\mathbf{X}_{\Phi \Phi}\right)_{l n}\left(\mathbf{X}_{\Phi \Phi}\right)_{n p}\left(\mathbf{X}_{\Phi \Phi}\right)_{p i} \\
& +\frac{1}{12} \tilde{\mathcal{I}}_{i k l n p r}^{111111}\left(\mathbf{X}_{\Phi \Phi}\right)_{i k}\left(\mathbf{X}_{\Phi \Phi}\right)_{k l}\left(\mathbf{X}_{\Phi \Phi}\right)_{l n}\left(\mathbf{X}_{\Phi \Phi}\right)_{n p}\left(\mathbf{X}_{\Phi \Phi}\right)_{p r}\left(\mathbf{X}_{\Phi \Phi}\right)_{r i} \\
& +\frac{1}{2} \tilde{\mathcal{I}}\left[q^{2}\right]_{k i}^{22}\left[P_{\mu},\left(\mathbf{X}_{\Phi \Phi}\right)_{i k}\right]\left[P^{\mu},\left(\mathbf{X}_{\Phi \Phi}\right)_{k i}\right] \\
& -\tilde{\mathcal{I}}\left[q^{2}\right]_{i l}^{21}\left(\mathbf{X}_{\Phi \Xi}\right)_{i l} \gamma^{\mu}\left[P_{\mu},\left(\mathbf{X}_{\Xi \Phi}\right)_{l i}\right] \\
& -\frac{1}{2} m_{\Xi_{k}} \tilde{\mathcal{I}}_{i k l}^{111}\left(\mathbf{X}_{\Phi \Xi}\right)_{i k}\left(\mathbf{X}_{\Xi \Phi}\right)_{k l}\left(\mathbf{X}_{\Phi \Phi}\right)_{l i}
\end{aligned}
$$

We furthermore set $P_{\mu} \equiv i \partial_{\mu}$ to omit contributions from gauge bosons. In our scenario we identify $\Sigma=\left(\tilde{t}_{L}, \tilde{t}_{R}\right)$ as the vector of (complex) heavy stops and $\Lambda=\tilde{g}^{a}$ as the heavy gluino. From (4.58) we then obtain the following non-vanishing derivatives

$$
\begin{aligned}
& \left(X_{\tilde{t}_{L}^{*} \tilde{t}_{L}}\right)_{i j}=\left(X_{\tilde{t}_{L} \tilde{t}_{L}^{*}}\right)_{i j}=\left(X_{\tilde{t}_{R}^{*} \tilde{t}_{R}}\right)_{i j}=\left(X_{\tilde{t}_{R} \tilde{t}_{R}^{*}}\right)_{i j}=\frac{1}{2}\left(y_{t} s_{\beta} h\right)^{2} \delta_{i j}, \\
& \left(X_{\tilde{t}_{L}^{*} \tilde{t}_{R}}\right)_{i j}=\left(X_{\tilde{t}_{L} \tilde{t}_{R}^{*}}\right)_{i j}=\left(X_{\tilde{t}_{R}^{*} \tilde{t}_{L}}\right)_{i j}=\left(X_{\tilde{t}_{R} \tilde{t}_{L}^{*}}\right)_{i j}=\frac{1}{\sqrt{2}} y_{t} s_{\beta} h X_{t} \delta_{i j},
\end{aligned}
$$




$$
\begin{aligned}
& \left(X_{\tilde{t}_{L} \tilde{g}^{a}}\right)_{i \alpha}^{a}=\left(X_{\tilde{g}^{a} \tilde{t}_{L}}\right)_{i \alpha}^{a}=-\sqrt{2} g_{3}\left(\bar{t}_{j} P_{R}\right)_{\alpha} T_{j i}^{a}, \\
& \left(X_{\tilde{t}_{R} \tilde{g}^{a}}\right)_{i \alpha}^{a}=\left(X_{\tilde{g}^{a} \tilde{t}_{R}}\right)_{i \alpha}^{a}=\sqrt{2} g_{3}\left(\bar{t}_{j} P_{L}\right)_{\alpha} T_{j i}^{a}, \\
& \left(X_{\tilde{g}^{a} \tilde{t}_{L}^{*}}\right)_{i \alpha}^{a}=\left(X_{\tilde{t}_{L}^{*} \tilde{g}^{a}}\right)_{i \alpha}^{a}=\sqrt{2} g_{3} T_{i j}^{a}\left(\mathcal{C} P_{L} t_{j}\right)_{\alpha}, \\
& \left(X_{\tilde{g}^{a} \tilde{t}_{R}^{*}}\right)_{i \alpha}^{a}=\left(X_{\tilde{t}_{R}^{*} \tilde{g}^{a}}\right)_{i \alpha}^{a}=-\sqrt{2} g_{3} T_{i j}^{a}\left(\mathcal{C} P_{R} t_{j}\right)_{\alpha},
\end{aligned}
$$

where $i, j=1,2,3$ and $a=1, \ldots, 8$ are color indices and $\alpha=1, \ldots, 4$ is a 4 -component spinor index. Note the flipped sign in eqs. (4.63)-(4.64) due to one anti-commutation of the spinor $\bar{t}$ with the derivative w.r.t. the spinor $\tilde{g}^{a}$. The bold derivative matrices thus become

$$
\begin{aligned}
& \mathbf{X}_{\Phi \Phi}=\left(\begin{array}{cc}
X_{\Sigma^{*} \Sigma} & X_{\Sigma^{*} \Sigma^{*}} \\
X_{\Sigma \Sigma} & X_{\Sigma \Sigma^{*}}
\end{array}\right)=\left(\begin{array}{cccc}
\left(X_{\tilde{t}_{L}^{*} \tilde{t}_{L}}\right)_{i j} & \left(X_{\tilde{t}_{L}^{*} \tilde{t}_{R}}\right)_{i j} & 0 & 0 \\
\left(X_{\tilde{t}_{R}^{*} \tilde{t}_{L}}\right)_{i j} & \left(X_{\tilde{t}_{R}^{*} \tilde{t}_{R}}\right)_{i j} & 0 & 0 \\
0 & 0 & \left(X_{\tilde{t}_{L} \tilde{t}_{L}^{*}}\right)_{i j} & \left(X_{\tilde{t}_{L} \tilde{t}_{R}^{*}}\right)_{i j} \\
0 & 0 & \left(X_{\tilde{t}_{R} \tilde{t}_{L}^{*}}\right)_{i j} & \left(X_{\tilde{t}_{R} \tilde{t}_{R}^{*}}\right)_{i j}
\end{array}\right) \\
& =\delta_{i j} \mathbf{1}_{2 \times 2} \otimes\left(\begin{array}{cc}
\frac{1}{2}\left(y_{t} s_{\beta} h\right)^{2} & \frac{1}{\sqrt{2}} y_{t} s_{\beta} h X_{t} \\
\frac{1}{\sqrt{2}} y_{t} s_{\beta} h X_{t} & \frac{1}{2}\left(y_{t} s_{\beta} h\right)^{2}
\end{array}\right) \text {, } \\
& \mathbf{X}_{\Phi \Xi}=\left(\begin{array}{c}
X_{\Sigma^{*} \Lambda} \\
X_{\Sigma \Lambda}
\end{array}\right)=\left(\begin{array}{c}
\left(X_{\tilde{t}_{L}^{*} \tilde{g}^{a}}\right)_{i \alpha}^{a} \\
\left(X_{\tilde{t}_{R}^{*} \tilde{g}^{a}}\right)_{i \alpha}^{a} \\
\left(X_{\tilde{t}_{L} \tilde{g}^{a}}\right)_{i \alpha}^{a} \\
\left(X_{\tilde{t}_{R} \tilde{g}^{a}}\right)_{i \alpha}^{a}
\end{array}\right)=\sqrt{2} g_{3}\left(\begin{array}{c}
T_{i j}^{a}\left(\mathcal{C} P_{L} t_{j}\right)_{\alpha} \\
-T_{i j}^{a}\left(\mathcal{C} P_{R} t_{j}\right)_{\alpha} \\
-\left(\bar{t}_{j} P_{R}\right)_{\alpha} T_{j i}^{a} \\
\left(\bar{t}_{j} P_{L}\right)_{\alpha} T_{j i}^{a}
\end{array}\right), \\
& \mathbf{X}_{\Xi \Phi}=\left(\mathcal{C}^{-1} X_{\Lambda \Sigma}, \mathcal{C}^{-1} X_{\Lambda \Sigma^{*}}\right) \\
& =\left(\mathcal{C}^{-1}\right)_{\alpha \beta}\left(\left(X_{\tilde{g}^{a} \tilde{t}_{L}}\right)_{i \beta}^{a}, \quad\left(X_{\tilde{g}^{a} \tilde{t}_{R}}\right)_{i \beta}^{a}, \quad\left(X_{\tilde{g}^{a} \tilde{t}_{L}^{*}}\right)_{i \beta}^{a}, \quad\left(X_{\tilde{g}^{a} \tilde{t}_{R}^{*}}\right)_{i \beta}^{a}\right) \\
& =\sqrt{2} g_{3}\left(\mathcal{C}^{-1}\right)_{\alpha \beta}\left(-\left(\bar{t}_{j} P_{R}\right)_{\beta} T_{j i}^{a}, \quad\left(\bar{t}_{j} P_{L}\right)_{\beta} T_{j i}^{a}, \quad T_{i j}^{a}\left(\mathcal{C} P_{L} t_{j}\right)_{\beta}, \quad-T_{i j}^{a}\left(\mathcal{C} P_{R} t_{j}\right)_{\beta}\right) \\
& =\sqrt{2} g_{3}\left(-\left(\bar{t}_{j} P_{R}\left(\mathcal{C}^{-1}\right)^{T}\right)_{\alpha} T_{j i}^{a}, \quad\left(\bar{t}_{j} P_{L}\left(\mathcal{C}^{-1}\right)^{T}\right)_{\alpha} T_{j i}^{a}, \quad T_{i j}^{a}\left(P_{L} t_{j}\right)_{\alpha},-T_{i j}^{a}\left(P_{R} t_{j}\right)_{\alpha}\right) .
\end{aligned}
$$

By inserting the $\mathbf{X}_{A B}$ operators into (4.60) and summing over all fields and colors we obtain

$$
\mathcal{L}_{\mathrm{EFT}}^{1 \ell}=c_{t} h \bar{t} t+c_{L} \bar{t} i \not \partial P_{L} t+c_{R} \bar{t} i \not \partial P_{R} t+c_{2}^{\prime}(\partial h)^{2}+c_{2} h^{2}+c_{4} h^{4}+c_{6} h^{6}+\cdots
$$

where

$$
\begin{aligned}
c_{t} & =-\frac{4 \sqrt{2}}{3} \kappa g_{3}^{2} y_{t} s_{\beta} m_{\tilde{g}} X_{t} \tilde{\mathcal{I}}_{\tilde{g} \tilde{q} \tilde{u}}^{111}, \\
c_{L} & =\frac{16}{3} \kappa g_{3}^{2} \tilde{\mathcal{I}}\left[q^{2}\right]_{\tilde{u} \tilde{g}}^{21}, \\
c_{R} & =\left.c_{L}\right|_{\tilde{q} \rightarrow \tilde{u}}, \\
c_{2}^{\prime} & =-3 \kappa\left(y_{t} s_{\beta}\right)^{2} X_{t}^{2} \tilde{\mathcal{I}}\left[q^{2}\right]_{\tilde{q} \tilde{u}}^{22}, \\
c_{2} & =\frac{3}{2} \kappa\left(y_{t} s_{\beta}\right)^{2}\left[\tilde{\mathcal{I}}_{\tilde{q}}^{1}+\tilde{\mathcal{I}}_{\tilde{u}}^{1}+X_{t}^{2} \tilde{\mathcal{I}}_{\tilde{q} \tilde{u}}^{11}\right],
\end{aligned}
$$




$$
\begin{aligned}
c_{4}= & \frac{3}{8} \kappa\left(y_{t} s_{\beta}\right)^{4}\left[\tilde{\mathcal{I}}_{\tilde{\tilde{q}} \tilde{q}}^{11}+\tilde{\mathcal{I}}_{\tilde{u} \tilde{u}}^{11}+2 X_{t}^{2}\left(\tilde{\mathcal{I}}_{\tilde{q} \tilde{q} \tilde{u}}^{11}+\tilde{\mathcal{I}}_{\tilde{q} \tilde{u} \tilde{u}}^{11}\right)+X_{t}^{4} \tilde{\mathcal{I}}_{\tilde{q} \tilde{q} \tilde{u} \tilde{u}}^{111}\right], \\
c_{6}= & \frac{1}{8} \kappa\left(y_{t} s_{\beta}\right)^{6}\left[\tilde{\mathcal{I}}_{\tilde{q} \tilde{q} \tilde{q}}^{111}+\tilde{\mathcal{I}}_{\tilde{u} \tilde{u} \tilde{u}}^{111}+3 X_{t}^{2}\left(\tilde{\mathcal{I}}_{\tilde{q} \tilde{q} \tilde{q} \tilde{u}}^{111}+\tilde{\mathcal{I}}_{\tilde{q} \tilde{q} \tilde{u} \tilde{u}}^{111}+\tilde{\mathcal{I}}_{\tilde{q} \tilde{u} \tilde{u} \tilde{u}}^{111}\right)\right. \\
& \left.+3 X_{t}^{4}\left(\tilde{\mathcal{I}}_{\tilde{q} \tilde{q} \tilde{q} \tilde{u} \tilde{u}}^{11111}+\tilde{\mathcal{I}}_{\tilde{q} \tilde{q} \tilde{u} \tilde{u} \tilde{u}}^{1111}\right)+X_{t}^{6} \tilde{\mathcal{I}}_{\tilde{q} \tilde{q} \tilde{q} \tilde{u} \tilde{u}}^{111}\right] .
\end{aligned}
$$

To canonically normalize the kinetic terms of $\mathcal{L}_{\text {SMEFT }}$ we re-define the Higgs and the top quark field as

$$
\begin{aligned}
h & =\left(1-\frac{1}{2} \delta Z_{h}\right) \hat{h}, \\
t_{L} & =\left(1-\frac{1}{2} \delta Z_{L}\right) \hat{t}_{L}, \\
t_{R} & =\left(1-\frac{1}{2} \delta Z_{R}\right) \hat{t}_{R},
\end{aligned}
$$

where the field renormalizations $\delta Z_{h / L / R}$ are given by

$$
\begin{aligned}
\delta Z_{h} & =2 c_{2}^{\prime}, \\
\delta Z_{L} & =c_{L}, \\
\delta Z_{R} & =c_{R} .
\end{aligned}
$$

If we parameterize the SMEFT Lagrangian as

$$
\mathcal{L}_{\mathrm{SMEFT}} \supset-\frac{g_{t}}{\sqrt{2}} \hat{h} \overline{\hat{t}} \hat{t}+\frac{m^{2}}{2} \hat{h}^{2}-\frac{\lambda}{8} \hat{h}^{4}-\frac{\tilde{c}_{6}}{8} \hat{h}^{6},
$$

then the SMEFT parameters $g_{t}, \lambda$ and $m^{2}$ are given by

$$
\begin{aligned}
g_{t} & =y_{t} s_{\beta}\left[1-\frac{1}{2}\left(c_{L}+c_{R}\right)-c_{2}^{\prime}-\frac{\sqrt{2} c_{t}}{y_{t} s_{\beta}}\right], \\
m^{2} & =2 c_{2} \\
\lambda & =-8 c_{4} \\
\tilde{c}_{6} & =-8 c_{6},
\end{aligned}
$$

which agrees with the results calculated in $[8,32,40,41] .{ }^{4}$

\subsection{Integrating out the gluino from the MSSM with light stops}

In this section we calculate some of the terms that arise when integrating out the gluino from the MSSM. This EFT scenario is relevant when there is a large hierarchy between the gluino mass and the stop masses in the MSSM. This example is also a direct application of most of the operators calculated in section 2.2, in particular operators where Majorana and Dirac fermions appear in loops at the same time.

\footnotetext{
${ }^{4}$ It was noted in [40] that the logarithmic term in the last line of eq. (D.4) in [8] should come with a minus sign.
} 
We consider the following part of the MSSM Lagrangian

$$
\begin{aligned}
\mathcal{L}_{\mathrm{MSSM}} \supset & \left|\partial \tilde{t}_{L}\right|^{2}-m_{\tilde{q}}^{2}\left|\tilde{t}_{L}\right|^{2}+\left|\partial \tilde{t}_{R}\right|^{2}-m_{\tilde{u}}^{2}\left|\tilde{t}_{R}\right|^{2}+\frac{1}{2}\left(\tilde{g}^{a}\right)^{T} \mathcal{C}\left(i \not \partial-m_{\tilde{g}}\right) \tilde{g}^{a} \\
& -\sqrt{2} g_{3}\left(\bar{t} P_{R} \tilde{g}^{a} T^{a} \tilde{t}_{L}-\bar{t} P_{L} \tilde{g}^{a} T^{a} \tilde{t}_{R}+\tilde{t}_{L}^{*}\left(\tilde{g}^{a}\right)^{T} T^{a} \mathcal{C} P_{L} t-\tilde{t}_{R}^{*}\left(\tilde{g}^{a}\right)^{T} T^{a} \mathcal{C} P_{R} t\right) \\
& +\left(-y_{t}^{2}+\frac{g_{3}^{2}}{2}\right)\left(\tilde{t}_{L}^{*} \tilde{t}_{R}\right)\left(\tilde{t}_{L} \tilde{t}_{R}^{*}\right)-\frac{g_{3}^{2}}{6}\left|\tilde{t}_{L}\right|^{2}\left|\tilde{t}_{R}\right|^{2}
\end{aligned}
$$

where we use the same notation as in section 4.4 with $t$ being the top quark, defined as a Dirac fermion, and $\tilde{g}^{a}=\left(\tilde{g}^{a}\right)^{C}$ denotes the gluino, which is a Majorana fermion. The complex scalar fields $\tilde{t}_{L}$ and $\tilde{t}_{R}$ represent the stops. In the following we determine the one-loop Wilson coefficients of the following operators in the EFT:

$$
\begin{aligned}
& \mathcal{L}_{\mathrm{EFT}}^{1 \ell} \supset c_{t_{L}} \bar{t}_{L} i \not t_{L}+c_{t_{R}} \bar{t}_{R} i \not \partial t_{R}+c_{\tilde{t}_{L}} \partial_{\mu} \tilde{t}_{L}^{*} \partial^{\mu} \tilde{t}_{L}-\left.\delta m_{\tilde{q}}^{2} \tilde{t}_{L}\right|^{2}+c_{\tilde{t}_{R}} \partial_{\mu} \tilde{t}_{R}^{*} \partial^{\mu} \tilde{t}_{R}-\delta m_{\tilde{u}}^{2}\left|\tilde{t}_{R}\right|^{2} \\
& +c_{41}^{L}\left(\tilde{t}_{L i}^{*} \tilde{t}_{L i}\right)^{2}+c_{42}^{L}\left(\tilde{t}_{L i}^{*} \tilde{t}_{L j}\right)\left(\tilde{t}_{L j}^{*} \tilde{t}_{L i}\right)+c_{4}^{R}\left(\tilde{t}_{R}^{*} \tilde{t}_{R}\right)^{2} \\
& +c_{41}^{L R}\left(\tilde{t}_{L i}^{*} \tilde{t}_{L i}\right)\left(\tilde{t}_{R j}^{*} \tilde{t}_{R j}\right)+c_{42}^{L R}\left(\tilde{t}_{L i}^{*} \tilde{t}_{L j}\right)\left(\tilde{t}_{R j}^{*} \tilde{t}_{R i}\right)+c_{G} G_{\mu \nu}^{a} G_{a}^{\mu \nu} \\
& +\left[c_{51}^{L L}\left(\bar{t}_{L i} T_{i j}^{a} \tilde{t}_{L j}\right)\left(t_{R k}^{C} T_{k l}^{a} \tilde{t}_{L l}\right)+c_{52}^{L L}\left(\tilde{t}_{L i}^{*} T_{i j}^{a} \overline{t_{R j}^{C}}\right)\left(\tilde{t}_{L k}^{*} T_{k l}^{a} t_{L l}\right)+(L \leftrightarrow R)\right] \\
& +\left[c_{51}^{L R}\left(\bar{t}_{L i} T_{i j}^{a} \tilde{t}_{L j}\right)\left(\tilde{t}_{R k}^{*} T_{k l}^{a} t_{R l}\right)+c_{52}^{L R}\left(\tilde{t}_{L i} \tilde{t}_{R i}^{*}\right)\left(\bar{t}_{L j} t_{R j}\right)+(L \leftrightarrow R)\right]+c_{61}^{L}\left(\tilde{t}_{L i}^{*} \tilde{t}_{L i}\right)^{3} \\
& +c_{62}^{L}\left(\tilde{t}_{L i}^{*} \tilde{t}_{L i}\right)\left(\tilde{t}_{L j}^{*} \tilde{t}_{L k}\right)\left(\tilde{t}_{L k}^{*} \tilde{t}_{L j}\right)+c_{63}^{L}\left(\tilde{t}_{L i}^{*} \tilde{t}_{L j}\right)\left(\tilde{t}_{L j}^{*} \tilde{t}_{L k}\right)\left(\tilde{t}_{L k}^{*} \tilde{t}_{L i}\right)+c_{6}^{R}\left(\tilde{t}_{R i}^{*} \tilde{t}_{R i}\right)^{3} \\
& +\left[c_{61}^{L R}\left(\tilde{t}_{L i}^{*} \tilde{t}_{L i}\right)^{2}\left(\tilde{t}_{R i}^{*} \tilde{t}_{R i}\right)+c_{62}^{L R}\left(\tilde{t}_{L i}^{*} \tilde{t}_{L i}\right)\left(\tilde{t}_{L j}^{*} \tilde{t}_{L k}\right)\left(\tilde{t}_{R k}^{*} \tilde{t}_{R j}\right)+c_{63}^{L R}\left(\tilde{t}_{L i}^{*} \tilde{t}_{L j}\right)\left(\tilde{t}_{L j}^{*} \tilde{t}_{L i}\right)\left(\tilde{t}_{R k}^{*} \tilde{t}_{R k}\right)\right. \\
& \left.+c_{64}^{L R}\left(\tilde{t}_{L i}^{*} \tilde{t}_{L j}\right)\left(\tilde{t}_{L j}^{*} \tilde{t}_{L k}\right)\left(\tilde{t}_{R k}^{*} \tilde{t}_{R i}\right)+c_{61}^{R L}\left(\tilde{t}_{R i}^{*} \tilde{t}_{R i}\right)^{2}\left(\tilde{t}_{L i}^{*} \tilde{t}_{L i}\right)+c_{62}^{R L}\left(\tilde{t}_{R i}^{*} \tilde{t}_{R i}\right)\left(\tilde{t}_{R j}^{*} \tilde{t}_{R k}\right)\left(\tilde{t}_{L k}^{*} \tilde{t}_{L j}\right)\right] \\
& +\left[c_{61}^{L^{\mu} L_{\mu}}\left(\bar{t}_{L i} \gamma^{\mu} t_{L i}\right)\left(\bar{t}_{L j} \gamma_{\mu} t_{L j}\right)+c_{62}^{L^{\mu} L_{\mu}}\left(\bar{t}_{L i} \gamma^{\mu} t_{L j}\right)\left(\bar{t}_{L j} \gamma_{\mu} t_{L i}\right)+(L \leftrightarrow R)\right] \\
& +c_{61}^{(L R)^{\mu}(R L)_{\mu}}\left(\overline{t_{R i}^{C}} \gamma^{\mu} t_{R j}\right)\left(\bar{t}_{R j} \gamma_{\mu} t_{R i}^{C}\right)+c_{62}^{(L R)^{\mu}(R L)_{\mu}}\left(\overline{t_{R j}^{C}} \gamma^{\mu} t_{R i}\right)\left(\bar{t}_{R j} \gamma_{\mu} t_{R i}^{C}\right) \\
& +\left[c_{61}^{L L}\left(\overline{t_{R i}^{C}} t_{L i}\right)\left(\bar{t}_{L j} t_{R j}^{C}\right)+c_{62}^{L L}\left(\overline{t_{R i}^{C}} t_{L j}\right)\left(\bar{t}_{L j} t_{R i}^{C}\right)+(L \leftrightarrow R)\right] \\
& +c_{61}^{(L R)(R L)}\left(\bar{t}_{R i} t_{L j}\right)\left(\bar{t}_{L j} t_{R i}\right)+c_{62}^{(L R)(R L)}\left(\bar{t}_{R j} t_{L i}\right)\left(\bar{t}_{L j} t_{R i}\right) \text {. }
\end{aligned}
$$

These operators represent all derived one-loop stop interactions in the gaugeless limit and in the unbroken phase, without contributions from higher-dimensional operators with covariant derivatives. Terms which involve SUSY particles beyond the stop are omitted for brevity. In (4.94) the color indices $i, j, k=1,2,3$ and $a=1, \ldots, 8$ are written out explicitly. Note that in general $\mathcal{L}_{\mathrm{EFT}}^{1 \ell}$ contains $\mathrm{SU}(2)_{L}$ and $\mathrm{SU}(3)_{C}$ invariant terms of the form $\left(\tilde{q}_{L i}^{\dagger} \tilde{q}_{L i}\right)\left(\tilde{q}_{L j}^{\dagger} \tilde{q}_{L j}\right)$ and $\left(\tilde{q}_{L i}^{\dagger} \tilde{q}_{L j}\right)\left(\tilde{q}_{L j}^{\dagger} \tilde{q}_{L i}\right)$, where the $\mathrm{SU}(2)_{L}$ indices are contracted within parentheses, but the color indices are contracted differently among the terms. In (4.94), however, the corresponding terms with the couplings $c_{41}^{L}$ and $c_{42}^{L}$ have the same structure, because we have omitted the sbottom quark.

The dimension 5 operators have contributions already at tree-level, which stem from the insertion of the gluino background field $\tilde{g}_{\mathrm{cl}}$ into the Lagrangian of the MSSM. The necessary part of the gluino background field can be extracted from the equation of motion

$$
\left[\mathcal{C}\left(i \not \partial-m_{\tilde{g}}\right)\right]_{\alpha \beta}\left(\tilde{g}_{\mathrm{cl}}\right)_{\beta}^{a}=\sqrt{2} g_{3}\left(-\bar{t}_{L \alpha} T^{a} \tilde{t}_{L}+\bar{t}_{R \alpha} T^{a} \tilde{t}_{R}+\tilde{t}_{L}^{*} T^{a}\left(\mathcal{C} t_{L}\right)_{\alpha}-\tilde{t}_{R}^{*} T^{a}\left(\mathcal{C} t_{R}\right)_{\alpha}\right)
$$


which yields

$$
\begin{aligned}
\left(\tilde{g}_{\mathrm{cl}}\right)_{\beta}^{a} & =\sqrt{2} g_{3}\left(i \not \partial-m_{\tilde{g}}\right)_{\beta \alpha}^{-1}\left[-\left(\bar{t}_{L} \mathcal{C}\right)_{\alpha} T^{a} \tilde{t}_{L}+\left(\bar{t}_{R} \mathcal{C}\right)_{\alpha} T^{a} \tilde{t}_{R}+\tilde{t}_{L}^{*} T^{a} t_{L \alpha}-\tilde{t}_{R}^{*} T^{a} t_{R \alpha}\right] \\
& =\frac{\sqrt{2} g_{3}}{m_{\tilde{g}}}\left[\left(\bar{t}_{L} \mathcal{C}\right)_{\beta} T^{a} \tilde{t}_{L}-\left(\bar{t}_{R} \mathcal{C}\right)_{\beta} T^{a} \tilde{t}_{R}-\tilde{t}_{L}^{*} T^{a} t_{L \beta}+\tilde{t}_{R}^{*} T^{a} t_{R \beta}+\cdots\right]
\end{aligned}
$$

where the ellipsis designate higher order terms of $\mathcal{O}\left(\partial / m_{\tilde{g}}\right)$ with at least one derivative. Inserting (4.97) into both the kinetic term of the gluino and the interaction Lagrangian one finds the tree-level values of $c_{5 i}^{A B}(A, B \in\{L, R\})$ to be

$$
\begin{aligned}
& c_{51}^{L L, \text { tree }}=c_{52}^{L L, \text { tree }}=c_{51}^{R R, \text { tree }}=c_{52}^{R R, \text { tree }}=\frac{g_{3}^{2}}{m_{\tilde{g}}}, \\
& c_{51}^{L R, \text { tree }}=c_{51}^{R L, \text { tree }}=-\frac{2 g_{3}^{2}}{m_{\tilde{g}}}, \\
& c_{52}^{L R \text {,tree }}=c_{52}^{R L, \text { tree }}=0 .
\end{aligned}
$$

At one-loop the relevant contributions from the UOLEA are

$$
\begin{aligned}
& \frac{1}{\kappa} \mathcal{L}_{\mathrm{EFT}}^{1 \ell}=\operatorname{tr}\left\{\left(-\tilde{\mathcal{I}}\left[q^{4}\right]_{\tilde{g} 0}^{31}+\frac{m_{\tilde{g}}^{2}}{12} \tilde{\mathcal{I}}\left[q^{2}\right] \tilde{g} 0\right) \gamma_{\mu}\left[P^{\nu},\left(\mathbf{X}_{\Xi \xi}\right)_{i}^{a}\right] \gamma^{\mu}\left[P_{\nu},\left(\mathbf{X}_{\xi \Xi}\right)_{i}^{a}\right]\right. \\
& +\left(-2 \tilde{\mathcal{I}}\left[q^{4}\right]_{\tilde{g} 0}^{31}+\frac{m_{\tilde{g}}^{2}}{6} \tilde{\mathcal{I}}\left[q^{2}\right]_{\tilde{g} 0}^{22}\right) \gamma_{\mu}\left[P^{\mu},\left(\mathbf{X}_{\Xi \xi}\right)_{i}^{a}\right] \gamma^{\nu}\left[P_{\nu},\left(\mathbf{X}_{\xi \Xi}\right)_{i}^{a}\right] \\
& +\left(-\tilde{\mathcal{I}}\left[q^{2}\right]_{\tilde{g} 0}^{12}-2 m_{\phi_{i}}^{2} \tilde{\mathcal{I}}\left[q^{2}\right]_{\tilde{g} 0}^{13}\right)\left(\mathbf{X}_{\phi \Xi}\right)_{i} \gamma^{\mu}\left[P_{\mu},\left(\mathbf{X}_{\Xi \phi}\right)_{i}\right] \\
& +\frac{1}{4} \tilde{\mathcal{I}}\left[q^{2}\right]_{\tilde{g} 0}^{22}\left(\mathbf{X}_{\phi \Xi}\right)_{i} \gamma^{\mu}\left(\mathbf{X}_{\Xi \phi}\right)_{j}\left(\mathbf{X}_{\phi \Xi}\right)_{j} \gamma_{\mu}\left(\mathbf{X}_{\Xi \phi}\right)_{i} \\
& -\frac{1}{2} m_{\tilde{g}} \tilde{\mathcal{I}}_{\tilde{g} 0}^{12}\left(\mathbf{X}_{\phi \phi}\right)_{i j}\left(\mathbf{X}_{\phi \Xi}\right)_{j}\left(\mathbf{X}_{\Xi \phi}\right)_{i} \\
& +\frac{1}{4} m_{\tilde{g}}^{2} \tilde{\mathcal{I}}_{\tilde{g} 0}^{22}\left(\mathbf{X}_{\phi \Xi}\right)_{i}\left(\mathbf{X}_{\Xi \phi}\right)_{j}\left(\mathbf{X}_{\phi \Xi}\right)_{j}\left(\mathbf{X}_{\Xi \phi}\right)_{i}-\frac{1}{2} \tilde{\mathcal{I}}\left[q^{2}\right]_{\tilde{g} 0}^{11} \gamma^{\mu}\left(\mathbf{X}_{\Xi \xi}\right)_{i} \gamma_{\mu}\left(\mathbf{X}_{\xi \Xi}\right)_{i} \\
& -\frac{1}{4} m_{\tilde{g}}^{2} \tilde{\mathcal{I}}\left[q^{2}\right]_{\tilde{g} 0}^{22}\left(\mathbf{X}_{\Xi \xi}\right)_{i}^{a} \gamma^{\mu}\left(\mathbf{X}_{\xi \Xi}\right)_{i}^{b}\left(\mathbf{X}_{\Xi \xi}\right)_{j}^{b} \gamma_{\mu}\left(\mathbf{X}_{\xi \Xi}\right)_{j}^{a} \\
& -\frac{1}{4} \tilde{\mathcal{I}}\left[q^{4}\right]_{\tilde{g} 0}^{22} g_{\mu \nu \rho \sigma}\left(\mathbf{X}_{\Xi \xi}\right)_{i}^{a} \gamma^{\mu}\left(\mathbf{X}_{\xi \Xi}\right)_{i}^{b} \gamma^{\nu}\left(\mathbf{X}_{\Xi \xi}\right)_{j}^{b} \gamma^{\rho}\left(\mathbf{X}_{\xi \Xi}\right)_{j}^{a} \gamma^{\sigma} \\
& -\frac{1}{2} m_{\tilde{g}}^{2} \tilde{\mathcal{I}}\left[q^{4}\right]_{\tilde{g} 0}^{33} g_{\mu \nu \rho \sigma}\left(\mathbf{X}_{\Xi \xi}\right)_{i}^{a} \gamma^{\mu}\left(\mathbf{X}_{\xi \Xi}\right)_{i}^{b}\left(\mathbf{X}_{\Xi \xi}\right)_{j}^{b} \gamma^{\nu}\left(\mathbf{X}_{\xi \Xi}\right)_{j}^{c} \gamma^{\rho}\left(\mathbf{X}_{\Xi \xi}\right)_{k}^{c} \gamma^{\sigma}\left(\mathbf{X}_{\xi \Xi}\right)_{k}^{a} \\
& -\frac{1}{6} \tilde{\mathcal{I}}\left[q^{6}\right]_{\tilde{g} 0}^{33} g_{\mu \nu \rho \sigma \kappa \lambda}\left(\mathbf{X}_{\Xi \xi}\right)_{i}^{a} \gamma^{\mu}\left(\mathbf{X}_{\xi \Xi}\right)_{i}^{b} \gamma^{\nu}\left(\mathbf{X}_{\Xi \xi}\right)_{j}^{b} \gamma^{\rho}\left(\mathbf{X}_{\xi \Xi}\right)_{j}^{c} \gamma^{\sigma}\left(\mathbf{X}_{\Xi \xi}\right)_{k}^{c} \gamma^{\kappa}\left(\mathbf{X}_{\xi \Xi}\right)_{k}^{a} \gamma^{\lambda} \\
& \left.+\frac{1}{6} \tilde{\mathcal{I}}_{\tilde{g}}^{2}\left[P_{\mu}, P_{\nu}\right]\left[P^{\mu}, P^{\nu}\right]\right\}
\end{aligned}
$$

where $g_{\mu \nu \ldots}$ is the combination of metric tensors which is totally symmetric in all indices, see appendix B. The derivatives with respect to the stops and the gluino have already been calculated in section 4.3 and are given by

$$
\mathbf{X}_{\phi \Xi}=\left(\begin{array}{c}
X_{\sigma^{*} \Lambda} \\
X_{\sigma \Lambda}
\end{array}\right)=\left(\begin{array}{c}
\left(X_{\tilde{t}_{L}^{*} \tilde{g}^{a}}\right)_{i \alpha}^{a} \\
\left(X_{\tilde{t}_{R}^{*} \tilde{g}^{a}}\right)_{i \alpha}^{a} \\
\left(X_{\tilde{t}_{L} \tilde{g}^{a}}\right)_{i \alpha}^{a} \\
\left(X_{\tilde{t}_{R} \tilde{g}^{a}}\right)_{i \alpha}^{a}
\end{array}\right)=\sqrt{2} g_{3}\left(\begin{array}{c}
T_{i j}^{a}\left(\mathcal{C} P_{L} t_{j}\right)_{\alpha} \\
-T_{i j}^{a}\left(\mathcal{C} P_{R} t_{j}\right)_{\alpha} \\
-\left(\bar{t}_{j} P_{R}\right)_{\alpha} T_{j i}^{a} \\
\left(\bar{t}_{j} P_{L}\right)_{\alpha} T_{j i}^{a}
\end{array}\right),
$$




$$
\begin{aligned}
\mathbf{X}_{\Xi \phi} & =\left(\mathcal{C}^{-1} X_{\Lambda \sigma}, \mathcal{C}^{-1} X_{\Lambda \sigma^{*}}\right) \\
& =\left(\mathcal{C}^{-1}\right)_{\alpha \beta}\left(\left(X_{\tilde{g}^{a} \tilde{t}_{L}}\right)_{i \beta}^{a},\left(X_{\tilde{g}^{a} \tilde{t}_{R}}\right)_{i \beta}^{a},\left(X_{\tilde{g}^{a} \tilde{t}_{L}^{*}}\right)_{i \beta}^{a},\left(X_{\tilde{g}^{a} \tilde{t}_{R}^{*}}\right)_{i \beta}^{a}\right) \\
& =\sqrt{2} g_{3}\left(-\left(\bar{t}_{j} P_{R} \mathcal{C}\right)_{\alpha} T_{j i}^{a},\left(\bar{t}_{j} P_{L} \mathcal{C}\right)_{\alpha} T_{j i}^{a}, T_{i j}^{a}\left(P_{L} t_{j}\right)_{\alpha},-T_{i j}^{a}\left(P_{R} t_{j}\right)_{\alpha}\right),
\end{aligned}
$$

the difference being that the stops are now considered to be light fields. For the purpose of this application we also need the derivatives with respect to a top and a gluino, which read

$$
\begin{aligned}
& \left(X_{\bar{t} \tilde{g}^{a}}\right)_{i \alpha \beta}^{a}=-\sqrt{2} g_{3} T_{i j}^{a}\left[\left(P_{R}\right)_{\alpha \beta} \tilde{t}_{L j}-\left(P_{L}\right)_{\alpha \beta} \tilde{t}_{R j}\right], \\
& \left(X_{t \tilde{g}^{a}}\right)_{i \alpha \beta}^{a}=-\sqrt{2} g_{3} T_{j i}^{a}\left[-\tilde{t}_{L j}^{*}\left(\mathcal{C} P_{L}\right)_{\beta \alpha}+\tilde{t}_{R j}^{*}\left(\mathcal{C} P_{R}\right)_{\beta \alpha}\right], \\
& \left(X_{\tilde{g}^{a} \bar{t}}\right)_{i \alpha \beta}^{a}=\sqrt{2} g_{3} T_{i j}^{a}\left[\left(P_{R}\right)_{\beta \alpha} \tilde{t}_{L j}-\left(P_{L}\right)_{\beta \alpha} \tilde{t}_{R j}\right], \\
& \left(X_{\tilde{g}^{a} t}\right)_{i \alpha \beta}^{a}=\sqrt{2} g_{3} T_{j i}^{a}\left[-\tilde{t}_{L j}^{*}\left(\mathcal{C} P_{L}\right)_{\alpha \beta}+\tilde{t}_{R j}^{*}\left(\mathcal{C} P_{R}\right)_{\alpha \beta}\right],
\end{aligned}
$$

and are collected into

$$
\begin{aligned}
\mathbf{X}_{\Xi \xi} & =\left(\mathcal{C}^{-1} X_{\Lambda \omega}, \mathcal{C}^{-1} X_{\Lambda \bar{\omega}} \mathcal{C}^{-1}\right) \\
& =\left(\left(\mathcal{C}^{-1} X_{\tilde{g}^{a} t}\right)_{i \alpha \beta}^{a},\left(\mathcal{C}^{-1} X_{\tilde{g}^{a} \bar{t}} \mathcal{C}^{-1}\right)_{i \alpha \beta}^{a}\right) \\
& =\left(-\sqrt{2} g_{3} T_{j i}^{a}\left[\tilde{t}_{L j}^{*}\left(P_{L}\right)_{\alpha \beta}-\tilde{t}_{R j}^{*}\left(P_{R}\right)_{\alpha \beta}\right],-\sqrt{2} g_{3} T_{i j}^{a}\left[\left(P_{R}\right)_{\alpha \beta} \tilde{t}_{L j}-\left(P_{L}\right)_{\alpha \beta} \tilde{t}_{R j}\right]\right), \\
\mathbf{X}_{\xi \Xi} & =\left(\begin{array}{c}
X_{\bar{\omega} \Lambda} \\
\mathcal{C}^{-1} X_{\omega \Lambda}
\end{array}\right)=\left(\begin{array}{c}
\left(X_{\bar{t} \tilde{g}^{a}}\right)_{i \alpha \beta}^{a} \\
\left(\mathcal{C}^{-1} X_{t \tilde{g}^{a}}\right)_{i \alpha \beta}^{a}
\end{array}\right)=\left(\begin{array}{l}
-\sqrt{2} g_{3} T_{i j}^{a}\left[\left(P_{R}\right)_{\alpha \beta} \tilde{t}_{L j}-\left(P_{L}\right)_{\alpha \beta} \tilde{t}_{R j}\right] \\
-\sqrt{2} g_{3} T_{j i}^{a}\left[\tilde{t}_{L j}^{*}\left(P_{L}\right)_{\alpha \beta}-\tilde{t}_{R j}^{*}\left(P_{R}\right)_{\alpha \beta}\right]
\end{array}\right) .
\end{aligned}
$$

Finally we give the derivatives with respect to two stops

$$
\begin{aligned}
\mathbf{X}_{\phi \phi} & =\left(\begin{array}{cc}
\mathbf{Y}_{\phi \phi} & \mathbf{0}_{2 \times 2} \\
\mathbf{0}_{2 \times 2} & \left(\mathbf{Y}_{\phi \phi}\right)^{*}
\end{array}\right), \\
\mathbf{Y}_{\phi \phi} & =\left(\begin{array}{ll}
x_{t} \tilde{t}_{R j}^{*} \tilde{t}_{R i}-\frac{g_{3}^{2}}{6} \tilde{t}_{R}^{*} \tilde{t}_{R} \delta_{i j} & x_{t} \delta_{i j} \tilde{t}_{L} \tilde{t}_{R}^{*}-\frac{g_{3}^{2}}{6} \tilde{t}_{L i} \tilde{t}_{R j}^{*} \\
x_{t} \delta_{i j} \tilde{t}_{L}^{*} \tilde{t}_{R}-\frac{g_{3}^{2}}{6} \tilde{t}_{R i} \tilde{t}_{L j}^{*} & x_{t} \tilde{t}_{L j}^{*} \tilde{t}_{L i}-\frac{g_{3}^{2}}{6} \tilde{t}_{L}^{*} \tilde{t}_{L} \delta_{i j}
\end{array}\right),
\end{aligned}
$$

where we have introduced the abbreviation $x_{t} \equiv y_{t}^{2}-g_{3}^{2} / 2$. Substituting these derivatives into (4.101) and summing over all indices one finds

$$
\begin{aligned}
c_{t_{L}} & =\frac{16}{3} g_{3}^{2}\left(\tilde{\mathcal{I}}\left[q^{2}\right]_{\tilde{g} 0}^{12}+2 m_{\tilde{q}}^{2} \tilde{\mathcal{I}}\left[q^{2}\right]_{\tilde{g} 0}^{13}\right), \\
c_{t_{R}} & =\frac{16}{3} g_{3}^{2}\left(\tilde{\mathcal{I}}\left[q^{2}\right]_{\tilde{g} 0}^{12}+2 m_{\tilde{u}}^{2} \tilde{\mathcal{I}}\left[q^{2}\right]_{\tilde{g} 0}^{13}\right), \\
c_{\tilde{t}_{L}} & =c_{\tilde{t}_{R}}=\frac{32}{3} g_{3}^{2}(d+2)\left(-\tilde{\mathcal{I}}\left[q^{4}\right]_{\tilde{g} 0}^{31}+\frac{m_{\tilde{q}}^{2}}{2} \tilde{\mathcal{I}}\left[q^{2}\right]_{\tilde{g} 0}^{22}\right), \\
c_{61}^{L^{\mu} L_{\mu}} & =c_{61}^{R^{\mu} R_{\mu}}=\frac{7}{6} g_{3}^{4} \tilde{\mathcal{I}}\left[q^{2}\right]_{\tilde{g} 0}^{22}, \\
c_{62}^{L^{\mu} L_{\mu}} & =c_{62}^{R^{\mu} R_{\mu}}=\frac{1}{18} g_{3}^{4} \tilde{\mathcal{I}}\left[q^{2}\right]_{\tilde{g} 0}^{22},
\end{aligned}
$$




$$
\begin{aligned}
& c_{61}^{(L R)^{\mu}(R L)_{\mu}}=\frac{10}{9} g_{3}^{4} \tilde{\mathcal{I}}\left[q^{2}\right]_{\tilde{g} 0}^{22}, \\
& c_{62}^{(L R)^{\mu}(R L)_{\mu}}=-\frac{2}{9} g_{3}^{4} \tilde{\mathcal{I}}\left[q^{2}\right]_{\tilde{g} 0}^{22}, \\
& c_{61}^{L L}=c_{61}^{R R}=\frac{5}{18} g_{3}^{4} m_{\tilde{g}}^{2} \tilde{\mathcal{I}}\left[q^{2}\right]_{\tilde{g} 0}^{22}, \\
& \left.c_{62}^{L L}=c_{62}^{R R}=-\frac{1}{6} g_{3}^{4} m_{\tilde{g}}^{2} \tilde{\mathcal{I}}\left[q^{2}\right]\right]_{\tilde{g} 0}^{22}, \\
& c_{61}^{(L R)(R L)}=\frac{7}{6} g_{3}^{4} m_{\tilde{g}}^{2} \tilde{\mathcal{I}}\left[q^{2}\right]_{\tilde{g} 0}^{22}, \\
& c_{62}^{(L R)(R L)}=\frac{1}{18} g_{3}^{4} m_{\tilde{g}}^{2} \tilde{\mathcal{I}}\left[q^{2}\right]_{\tilde{g} 0}^{22}, \\
& \delta m_{\tilde{q}}^{2}=\delta m_{\tilde{u}}^{2}=\frac{16}{3} d g_{3}^{2} \tilde{\mathcal{I}}\left[q^{2}\right]_{\tilde{g} 0}^{11}, \\
& c_{41}^{L}=-\frac{40}{9} m_{\tilde{g}}^{2} g_{3}^{4} \tilde{\mathcal{I}}\left[q^{2}\right]_{\tilde{g} 0}^{22}-\frac{1}{9} d(d+2) g_{3}^{4} \tilde{\mathcal{I}}\left[q^{4}\right]_{\tilde{g} 0}^{22}, \\
& c_{4}^{R}=-\frac{16}{3} m_{\tilde{g}}^{2} g_{3}^{4} \tilde{\mathcal{I}}\left[q^{2}\right]_{\tilde{g} 0}^{22}-\frac{22}{9} d(d+2) g_{3}^{4} \tilde{\mathcal{I}}\left[q^{4}\right]_{\tilde{g} 0}^{22}, \\
& c_{42}^{L}=\frac{8}{3} m_{\tilde{g}}^{2} g_{3}^{4} \tilde{\mathcal{I}}\left[q^{2}\right]_{\tilde{g} 0}^{22}-\frac{7}{3} d(d+2) g_{3}^{4} \tilde{\mathcal{I}}\left[q^{4}\right]_{\tilde{g} 0}^{22}, \\
& c_{41}^{L R}=-\frac{8}{9} m_{\tilde{g}}^{2} g_{3}^{4} \tilde{\mathcal{I}}\left[q^{2}\right]_{\tilde{g} 0}^{22}-\frac{20}{9} d(d+2) g_{3}^{4} \tilde{\mathcal{I}}\left[q^{4}\right]_{\tilde{g} 0}^{22}, \\
& c_{42}^{L R}=-\frac{56}{3} m_{\tilde{g}}^{2} g_{3}^{4} \tilde{\mathcal{I}}\left[q^{2}\right]_{\tilde{g} 0}^{22}+\frac{4}{9} d(d+2) g_{3}^{4} \tilde{\mathcal{I}}\left[q^{4}\right]_{\tilde{g} 0}^{22}, \\
& c_{61}^{L}=\frac{1}{54} d(d+2) g_{3}^{6} m_{\tilde{g}}^{2} \tilde{\mathcal{I}}\left[q^{4}\right]_{\tilde{g} 0}^{33}+\frac{2}{81} d\left(d^{2}+6 d+8\right) g_{3}^{6} \tilde{\mathcal{I}}\left[q^{6}\right]_{\tilde{g} 0}^{33}, \\
& c_{62}^{L}=-\frac{2}{3} d(d+2) g_{3}^{6} m_{\tilde{g}}^{2} \tilde{\mathcal{I}}\left[q^{4}\right]_{\tilde{g} 0}^{33}-\frac{2}{9} d\left(d^{2}+6 d+8\right) g_{3}^{6} \tilde{\mathcal{I}}\left[q^{6}\right]_{\tilde{g} 0}^{33}, \\
& c_{63}^{L}=\frac{1}{2} d(d+2) g_{3}^{6} m_{\tilde{g}}^{2} \tilde{\mathcal{I}}\left[q^{4}\right]_{\tilde{g} 0}^{33}-\frac{4}{3} d\left(d^{2}+6 d+8\right) g_{3}^{6} \tilde{\mathcal{I}}\left[q^{6}\right]_{\tilde{g} 0}^{33}, \\
& c_{6}^{R}=-\frac{4}{27} d(d+2) g_{3}^{6} m_{\tilde{g}}^{2} \tilde{\mathcal{I}}\left[q^{4}\right]_{\tilde{g} 0}^{33}-\frac{124}{81} d\left(d^{2}+6 d+8\right) g_{3}^{6} \tilde{\mathcal{I}}\left[q^{6}\right]_{\tilde{g} 0}^{33}, \\
& c_{61}^{L R}=\frac{1}{18} d(d+2) g_{3}^{6} m_{\tilde{g}}^{2} \tilde{\mathcal{I}}\left[q^{4}\right]_{\tilde{g} 0}^{33}+\frac{2}{27} d\left(d^{2}+6 d+8\right) g_{3}^{6} \tilde{\mathcal{I}}\left[q^{6}\right]_{\tilde{g} 0}^{33}, \\
& c_{62}^{L R}=-\frac{12}{9} d(d+2) g_{3}^{6} m_{\tilde{g}}^{2} \tilde{\mathcal{I}}\left[q^{4}\right]_{\tilde{g} 0}^{33}-\frac{10}{9} d\left(d^{2}+6 d+8\right) g_{3}^{6} \tilde{\mathcal{I}}\left[q^{6}\right]_{\tilde{g} 0}^{33}, \\
& c_{63}^{L R}=-\frac{1}{6} d(d+2) g_{3}^{6} m_{\tilde{g}}^{2} \tilde{\mathcal{I}}\left[q^{4}\right]_{\tilde{g} 0}^{33}-\frac{14}{9} d\left(d^{2}+6 d+8\right) g_{3}^{6} \tilde{\mathcal{I}}\left[q^{6}\right]_{\tilde{g} 0}^{33}, \\
& c_{64}^{L R}=\frac{2}{9} d\left(d^{2}+6 d+8\right) g_{3}^{6} \tilde{\mathcal{I}}\left[q^{6}\right]_{\tilde{g} 0}^{33} \text {, } \\
& c_{61}^{R L}=-\frac{1}{9} d(d+2) g_{3}^{6} m_{\tilde{g}}^{2} \tilde{\mathcal{I}}\left[q^{4}\right]_{\tilde{g} 0}^{33}-\frac{40}{27} d\left(d^{2}+6 d+8\right) g_{3}^{6} \tilde{\mathcal{I}}\left[q^{6}\right]_{\tilde{g} 0}^{33}, \\
& \left.c_{62}^{R L}=-\frac{12}{9} d(d+2) g_{3}^{6} m_{\tilde{g}}^{2} \tilde{\mathcal{I}}\left[q^{4}\right]_{\tilde{g} 0}^{33}+\frac{8}{9} d\left(d^{2}+6 d+8\right) g_{3}^{6} \tilde{\mathcal{I}}\left[q^{6}\right]\right]_{\tilde{g} 0}^{33}, \\
& c_{51}^{L R, 1 \ell}=c_{51}^{R L, 1 \ell}=-\frac{g_{3}^{4}}{3} m_{\tilde{g}} \tilde{\mathcal{I}}_{\tilde{g} 0}^{12},
\end{aligned}
$$




$$
\begin{aligned}
c_{52}^{L R, 1 \ell} & =c_{52}^{R L, 1 \ell}=-\frac{8}{3} g_{3}^{4} x_{t} m_{\tilde{g}} \tilde{\mathcal{I}}_{\tilde{g} 0}^{12}, \\
c_{G} & =-\frac{g_{3}^{2}}{2} \tilde{\mathcal{I}}_{\tilde{g}}^{2} .
\end{aligned}
$$

In the calculation of these corrections the relations $g^{\mu \nu} g_{\mu \nu}=d=4-\epsilon$ and (C.12) were used repeatedly. The one-loop corrections $\delta m_{\tilde{q}}^{2}$ and $\delta m_{\tilde{u}}^{2}$ to the third generation squark mass parameters have already been calculated in [42] and our results agree with the expressions found there.

Since supersymmetry is only softly broken in the MSSM it is convenient to use DRED as a regulator. Once the gluino is integrated out from the theory, supersymmetry is explicitly broken and it is natural to regularize the EFT in DREG. This switch in the regularization scheme introduces further contributions to the couplings of the EFT coming from the epsilon scalars. In the formalism of the UOLEA the relevant operators which contribute here are given by [10]

$$
\begin{aligned}
\frac{\epsilon}{\kappa} \mathcal{L}_{\text {reg }}^{1 \ell}= & -\sum_{i}\left(m_{\epsilon}^{2}\right)_{i}\left(\breve{X}_{\epsilon \epsilon \mu}^{\mu}\right)_{i i}+\frac{1}{2} \sum_{i j}\left(\breve{X}_{\epsilon \epsilon \nu}^{\mu}\right)_{i j}\left(\breve{X}_{\epsilon \epsilon \mu}^{\nu}\right)_{j i} \\
& +\sum_{i j} 2^{c_{F_{j}}}\left\{2 m_{\psi j}\left(\breve{X}_{\epsilon \psi}^{\mu}\right)_{i j}\left(\breve{X}_{\bar{\psi} \epsilon \mu}\right)_{j i}+\left(\breve{X}_{\epsilon \psi}^{\mu}\right)_{i j} \gamma^{\nu}\left[P_{\nu},\left(\breve{X}_{\bar{\psi} \epsilon \mu}\right)_{j i}\right]\right\} \\
& -\sum_{i j k} 2^{c_{F_{j}}+c_{F_{k}}-1}\left(\breve{X}_{\epsilon \psi}^{\mu}\right)_{i j} \gamma^{\nu}\left(X_{\bar{\psi} \psi}\right)_{j k} \gamma_{\nu}\left(\breve{X}_{\bar{\psi} \epsilon \mu}\right)_{k i} \\
& +\frac{\epsilon}{12} \operatorname{tr}\left[G_{\mu \nu}^{\prime} G^{\prime \mu \nu}\right],
\end{aligned}
$$

The $\breve{X}$ operators are projections of the corresponding 4-dimensional ones ${ }_{X}^{\circ}$ onto the $\epsilon$ dimensional $Q \epsilon S$ space, i.e.

$$
\begin{aligned}
\breve{X}^{\mu} & =\breve{g}_{\sigma}^{\mu} \stackrel{\circ}{X}^{\sigma}, \\
\breve{X}^{\mu \nu} & =\breve{g}_{\sigma}^{\mu} \breve{g}_{\rho}^{\nu} \stackrel{\stackrel{X}{ }}{ }^{\sigma \rho},
\end{aligned}
$$

see appendix D. Furthermore, $G_{\mu \nu}^{\prime}=-i g_{3} G_{\mu \nu}^{a} T^{a}$ is the gluon field strength tensor. For the top quark (a Dirac fermion) we have $c_{F}=0$, and for the gluino (a Majorana fermion) $c_{F}=1$. From (4.146) we obtain the following additional contributions to the couplings of the EFT

$$
\begin{aligned}
\left(\delta m_{\tilde{q}}^{2}\right)_{\epsilon} & =\left(\delta m_{\tilde{u}}^{2}\right)_{\epsilon}=-\frac{4}{3} g_{3}^{2} m_{\epsilon}^{2}, \\
\left(c_{t_{L}}\right)_{\epsilon} & =\left(c_{t_{R}}\right)_{\epsilon}=\frac{4}{3} g_{3}^{2}, \\
\left(c_{41}^{L}\right)_{\epsilon} & =\frac{1}{72} g_{3}^{4}, \\
\left(c_{42}^{L}\right)_{\epsilon} & =\frac{7}{24} g_{3}^{4}, \\
\left(c_{4}^{R}\right)_{\epsilon} & =\frac{11}{36} g_{3}^{4}, \\
\left(c_{41}^{L R}\right)_{\epsilon} & =\frac{1}{36} g_{3}^{4},
\end{aligned}
$$




$$
\begin{aligned}
\left(c_{42}^{L R}\right)_{\epsilon} & =\frac{7}{12} g_{3}^{4}, \\
\left(c_{51}^{L L}\right)_{\epsilon} & =\left(c_{52}^{L L}\right)_{\epsilon}=\left(c_{51}^{R R}\right)_{\epsilon}=\left(c_{52}^{R R}\right)_{\epsilon}=\frac{3 g_{3}^{4}}{2 m_{\tilde{g}}} d, \\
\left(c_{51}^{L R}\right)_{\epsilon} & =\left(c_{52}^{R L}\right)_{\epsilon}=-\frac{3 g_{3}^{4}}{m_{\tilde{g}}} d, \\
\left(c_{G}\right)_{\epsilon} & =-\frac{g_{3}^{2}}{4} .
\end{aligned}
$$

The term $\propto m_{\epsilon}^{2}$ on the r.h.s. of (4.149) can be removed by switching from the $\overline{\mathrm{DR}}$ to the $\overline{\mathrm{DR}}^{\prime}$ scheme [43], which involves shifting $m_{\tilde{q}}^{2}$ and $m_{\tilde{u}}^{2}$ by finite terms. Notice also that the one-loop DRED-DREG conversion corrections to the coefficients of the dimension 5 operators arise from the third line of (4.146), which among other terms contains the term

$$
\left(\breve{X}_{\epsilon t}^{\mu}\right) \gamma^{\nu}\left(X_{\bar{t} \tilde{g}}\right) \gamma_{\nu}\left(\breve{X}_{\bar{g} \epsilon \mu}\right)
$$

Here $\left(\breve{X}_{\tilde{\tilde{g}} \epsilon \mu}\right)$ has an explicit dependence on the gluino spinor $\tilde{g}$,

$$
\left(\breve{X}_{\overline{\tilde{g}} \epsilon \mu}\right)^{b a}=\frac{i g_{3}}{2} \breve{\gamma}^{\mu} f^{a b c} \tilde{g}^{c}
$$

which must be eliminated by inserting the background field from (4.97). As noted above the threshold corrections for the two stop masses agree with the results derived in [42] when the effect of the sbottom quarks is neglected.

\section{Conclusions}

In this paper we have presented an extension of the Universal One-Loop Effective Action (UOLEA) by all one-loop operators up to dimension 6 for generic theories with scalar and fermionic fields, excluding operators stemming from open covariant derivatives in the UV Lagrangian. Our generic results can be used to derive the analytic expressions of all one-loop Wilson coefficients up to dimension 6 of an effective Lagrangian from a given UV theory with heavy scalar or fermionic particles, as long as second derivatives of the UV Lagrangian w.r.t. the fields do not contain covariant derivatives. Thus, our new results allow for an application of the UOLEA to a broader class of UV models than before.

To illustrate and test our generic results we have applied the UOLEA to different EFTs of the SM and the MSSM, where parts of the spectrum are heavy. We were able to reproduce known results from the literature, including the prediction of some one-loop Wilson coefficients of higher-dimensional operators of the SMEFT.

We have published our results in form of the two Mathematica files UOLEA.m and LoopFunctions.m provided in the arXiv submission, which allow for a direct use of our expressions and a potential implementation into generic tools such as CoDEx or spectrum generator generators such as SARAH and FlexibleSUSY. 


\section{Acknowledgments}

We kindly thank Jérémie Quevillon for helpful discussions regarding the UOLEA. BS would like to thank the Institute for Theoretical Physics in Heidelberg, where part of this work was completed, for its hospitality. This research was supported by the German DFG Collaborative Research Centre $P^{3} H$ : Particle Physics Phenomenology after the Higgs Discovery (CRC TRR 257).

\section{A Fermionic shifts}

In this section we discuss the consistency of the shift (2.33). The treatment of the shift given in (2.40) is analogous but somewhat more involved. Since $\xi$ is a multiplet of Majorana-like component spinors, for the shift

$$
\delta \xi^{\prime}=\delta \xi+\boldsymbol{\Delta}_{\xi}^{-1}\left[\tilde{\mathbf{X}}_{\xi \Xi} \delta \Xi-\tilde{\mathbf{X}}_{\xi \Phi} \delta \Phi-\tilde{\mathbf{X}}_{\xi \phi} \delta \phi\right]
$$

to be consistent it is necessary and sufficient that

$$
\left(\boldsymbol{\Delta}_{\xi}^{-1}\left[\tilde{\mathbf{X}}_{\xi \Xi} \delta \Xi-\tilde{\mathbf{X}}_{\xi \Phi} \delta \Phi-\tilde{\mathbf{X}}_{\xi \phi} \delta \phi\right]\right)^{T}=\left[\delta \Xi^{T} \tilde{\mathbf{X}}_{\Xi \xi}+\delta \Phi^{T} \tilde{\mathbf{X}}_{\Phi \xi}+\delta \phi^{T} \tilde{\mathbf{X}}_{\phi \xi}\right] \overleftarrow{\boldsymbol{\Delta}}_{\xi}^{-1}
$$

In the following we show that (A.2) holds. We first construct $\boldsymbol{\Delta}_{\xi}^{-1}$ in position space through its Neumann series ${ }^{5}$

$$
\begin{aligned}
\boldsymbol{\Delta}_{\xi}^{-1}(x, y) & =\sum_{n=0}^{\infty}\left(\prod_{\substack{i=1 \\
n>0}}^{n} \int \mathrm{d}^{d} x_{i} \mathbf{S}\left(x_{i-1}, x_{i}\right)\left(-\mathbf{X}_{\xi \xi}\left(x_{i}\right)\right)\right) \mathbf{S}\left(x_{n}, y\right) \tilde{\mathbb{1}} \mathcal{C}^{-1} \\
& \equiv \sum_{n=0}^{\infty}\left(\prod_{\substack{i=1 \\
n>0}}^{n} \mathbf{S}_{x_{i-1} x_{i}}\left(-\mathbf{X}_{\xi \xi x_{i}}\right)\right) \mathbf{S}_{x_{n} y} \tilde{\mathbb{1}} \mathcal{C}^{-1}
\end{aligned}
$$

where $x_{0} \equiv x$ and $\mathbf{S}(x, y)$ is the matrix-valued Green's function for $\left(\not P-M_{\xi}\right)$, which itself can be expressed through a Neumann series. To keep expressions short we also introduced the convention of denoting space-time points by indices, where repeated indices are integrated over. We may write $\left(\not P-M_{\xi}\right)=\left(i \not \partial-M_{\xi}-\mathbf{A}\right)$ with

$$
\mathbf{A}=i \sum_{j} g_{j} A_{j}^{a} T_{j}^{a}
$$

where we sum over all factors of the gauge group for a direct product group and $T_{j}^{a}$ is a block-diagonal matrix which generates the reducible representation of $\xi$. Due to the fact that $\xi$ contains $\omega, \omega^{C}$ and $\lambda$ (see table 1), the generator is of the form

$$
T^{a}=\left(\begin{array}{ccc}
T_{R(\omega)}^{a} & 0 & 0 \\
0 & T_{\bar{R}(\omega)}^{a} & 0 \\
0 & 0 & T_{R(\lambda)}^{a}
\end{array}\right),
$$

\footnotetext{
${ }^{5}$ In what follows we always write the whole series. In practice, however, we are only ever interested in a finite number of terms with all higher order terms being suppressed by higher powers of couplings.
} 
where $R(\omega)$ is the representation under which $\omega$ transforms, $\bar{R}(\omega)$ its conjugate representation and $R(\lambda)$ is the representation of $\lambda$, which is necessarily real. We then have

$$
\mathbf{S}_{x y}=\sum_{k=0}^{\infty}\left(\prod_{\substack{i=1 \\ k>0}}^{k} \mathbf{S}_{f, x_{i-1} x_{i}} \mathbf{A}_{x_{i}}\right) \mathbf{S}_{f, x_{k} y}
$$

where again $x_{0} \equiv x$ and $\mathbf{S}_{f, x y}$ is the matrix containing the Green's function of the free Dirac equation on its diagonal. It can be verified by explicit calculation that

$$
\mathbf{S}_{x y}\left(-i \overleftarrow{\not \partial_{y}}-M_{\xi}-\mathbf{A}_{y}\right)=\delta_{x y}
$$

which means that

$$
\boldsymbol{\Delta}_{\xi, x y}^{-1} \overleftarrow{\Delta}_{\xi, y}=\delta_{x y}
$$

and therefore $\overleftarrow{\Delta}_{\xi, y x}^{-1}=\boldsymbol{\Delta}_{\xi, y x}^{-1}$. Hence (A.2) reads

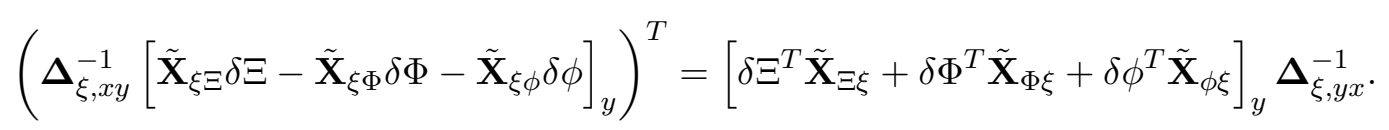

It is then useful to calculate

$$
\begin{aligned}
\mathcal{C} \tilde{\mathbb{1}} \mathbf{S}_{x y}^{T} & =\mathcal{C} \tilde{\mathbb{1}} \sum_{k=0}^{\infty} \mathbf{S}_{f, x_{k} y}^{T}\left(\prod_{\substack{i=k \\
k>0}}^{1} \mathbf{A}_{x_{i}}^{T} \mathbf{S}_{f, x_{i-1} x_{i}}^{T}\right) \\
& =\mathcal{C} \tilde{\mathbb{1}} \sum_{k=0}^{\infty} \mathcal{C} \mathbf{S}_{f, y x_{k}} \mathcal{C}^{-1}\left(\prod_{\substack{i=k \\
k>0}}^{1} \mathbf{A}_{x_{i}}^{T} \mathcal{C} \mathbf{S}_{f, x_{i} x_{i-1}} \mathcal{C}^{-1}\right) \\
& =-\sum_{k=0}^{\infty} \tilde{\mathbb{1}} \mathbf{S}_{f, y x_{k}} \tilde{\mathbb{1}} \tilde{\mathbb{1}}\left(\prod_{\substack{i=k \\
k>0}}^{1}\left(-\mathbf{A}_{x_{i}}^{t}\right) \tilde{\mathbb{1}} \tilde{\mathbb{1}} \mathbf{S}_{f, x_{i} x_{i-1}} \tilde{\mathbb{1}} \tilde{\mathbb{1}}\right) \mathcal{C}^{-1} \\
& =-\sum_{k=0}^{\infty} \mathbf{S}_{f, y x_{k}}\left(\prod_{\substack{i=k \\
k>0}}^{1}\left(-\tilde{\mathbb{1}} \mathbf{A}_{x_{i}}^{t} \tilde{\mathbb{1}}\right) \mathbf{S}_{f, x_{i} x_{i-1}}\right) \tilde{\mathbb{1}} \mathcal{C}^{-1} \\
& =-\sum_{k=0}^{\infty} \mathbf{S}_{f, y x_{k}}\left(\prod_{\substack{i=k \\
k>0}}^{1} \mathbf{A}_{x_{i}} \mathbf{S}_{f, x_{i} x_{i-1}}\right) \tilde{\mathbb{1} \mathcal{C}^{-1}} \\
& =-\mathbf{S}_{y x} \tilde{\mathbb{1}} \mathcal{C}^{-1},
\end{aligned}
$$

where $\mathbf{A}^{t}$ means taking the transpose of the gauge group generators only and we used that

$$
\tilde{\mathbb{1}}\left(\begin{array}{ccc}
A & 0 & 0 \\
0 & B & 0 \\
0 & 0 & C
\end{array}\right) \tilde{\mathbb{1}}=\left(\begin{array}{ccc}
B & 0 & 0 \\
0 & A & 0 \\
0 & 0 & C
\end{array}\right)
$$


We then find

$$
\begin{aligned}
\left(\boldsymbol{\Delta}_{\xi, x y}^{-1}\right)^{T} & =\mathcal{C} \tilde{\mathbb{1}} \sum_{n=0}^{\infty} \mathbf{S}_{x_{n}, y}^{T}\left(\prod_{\substack{i=1 \\
n>0}}^{n}\left(-\mathbf{X}_{\xi \xi, x_{i}}\right)^{T} \mathbf{S}_{x_{i-1} x_{i}}^{T}\right) \\
& =\sum_{n=0}^{\infty} \mathbf{S}_{y x_{n}} \tilde{\mathbb{1}} \mathcal{C}\left(\prod_{\substack{i=1 \\
n>0}}^{n}\left(-\mathbf{X}_{\xi \xi, x_{i}}\right)^{T} \mathbf{S}_{x_{i-1} x_{i}}^{T}\right) \\
& =\sum_{n=0}^{\infty} \mathbf{S}_{y x_{n}} \tilde{\mathbb{1}} \mathcal{C}\left(\prod_{\substack{i=1 \\
n>0}}^{n}\left(-\mathbf{X}_{\xi \xi, x_{i}}\right)^{T} \tilde{\mathbb{1}} \mathcal{C}^{-1} \tilde{\mathbb{1}} \mathcal{C} \mathbf{S}_{x_{i-1} x_{i}}^{T} \tilde{\mathbb{1}} \mathcal{C}^{-1} \tilde{\mathbb{1} \mathcal{C}}\right) \\
& =\sum_{n=0}^{\infty} \mathbf{S}_{y x_{n}} \tilde{\mathbb{1}} \mathcal{C}\left(\prod_{\substack{i=1 \\
n>0}}^{n}\left(-\mathbf{X}_{\xi \xi, x_{i}}\right)^{T} \tilde{\mathbb{1}} \mathcal{C}^{-1} \mathbf{S}_{x_{i} x_{i-1}} \tilde{\mathbb{C}}\right) \\
& =-\sum_{n=0}^{\infty} \mathbf{S}_{y x_{n}}\left(\prod_{\substack{i=1 \\
n>0}}^{n}\left(-\mathbf{X}_{\xi \xi, x_{i}}\right) \mathbf{S}_{x_{i} x_{i-1}}\right) \tilde{\mathbb{1} \mathcal{C}^{-1}} \\
& =-\boldsymbol{\Delta}_{\xi y x}^{-1}, \quad
\end{aligned}
$$

where we used that

$$
\mathcal{C} \tilde{\mathbb{1}} \mathbf{X}_{\xi \xi}^{T} \tilde{\mathbb{1}} \mathcal{C}^{-1}=\mathbf{X}_{\xi \xi}
$$

Noting that

$$
\begin{aligned}
\tilde{\mathbf{X}}_{\xi \Xi}^{T} & =-\tilde{\mathbf{X}}_{\Xi \xi}, \\
\tilde{\mathbf{X}}_{\xi \Phi}^{T} & =\tilde{\mathbf{X}}_{\Phi \xi}, \\
\tilde{\mathbf{X}}_{\xi \phi}^{T} & =\tilde{\mathbf{X}}_{\phi \xi},
\end{aligned}
$$

the validity of (A.2) follows immediately.

\section{B Loop functions}

The integrals $\tilde{\mathcal{I}}\left[q^{2 n_{c}}\right]_{i j \ldots 0}^{n_{i} n_{j} \ldots n_{L}}$ are defined as in [18], that is

$$
\int \frac{\mathrm{d}^{d} q}{(2 \pi)^{d}} \frac{q^{\mu_{1}} q^{\mu_{2}} \ldots q^{\mu_{2 n_{c}}}}{\left(q^{2}-M_{i}^{2}\right)^{n_{i}}\left(q^{2}-M_{j}^{2}\right)^{n_{j}} \ldots\left(q^{2}\right)^{n_{L}}} \equiv \frac{i}{16 \pi^{2}} g^{\mu_{1} \mu_{2} \ldots \mu_{2 n_{c}} \tilde{\mathcal{I}}}\left[q^{2 n_{c}}\right]_{i j \ldots 0}^{n_{i} n_{j} \ldots n_{L}},
$$

where $g^{\mu_{1} \mu_{2} \ldots \mu_{2 n_{c}}}$ is the completely symmetric combination of metric tensors with $2 n_{c}$ indices, for instance $g^{\mu \nu \rho \sigma}=g^{\mu \nu} g^{\rho \sigma}+g^{\mu \rho} g^{\nu \sigma}+g^{\mu \sigma} g^{\nu \rho}$. For $n_{c}=0$ we define the shorthand notation $\tilde{\mathcal{I}}\left[q^{0}\right]_{i j \ldots 0}^{n_{i} n_{j} \ldots n_{L}} \equiv \tilde{\mathcal{I}}_{i j \ldots 0}^{n_{i} n_{j} \ldots n_{L}}$. The integrals can be reduced to basis integrals using the reduction relations [18]

$$
\begin{aligned}
& \tilde{\mathcal{I}}\left[q^{2 n_{c}}\right]_{i j \ldots 0}^{n_{i} n_{j} \ldots n_{L}}=\frac{1}{\Delta_{i j}^{2}}\left(\tilde{\mathcal{I}}\left[q^{2 n_{c}}\right]^{n_{i} n_{j}-1 \ldots n_{L}}-\tilde{\mathcal{I}}\left[q^{2 n_{c}}\right]^{n_{i}-1 n_{j} \ldots n_{L}}\right), \\
& \tilde{\mathcal{I}}\left[q^{2 n_{c}}\right]_{i j \ldots 0}^{n_{i} n_{j} \ldots n_{L}}=\frac{1}{M_{i}^{2}}\left(\tilde{\mathcal{I}}\left[q^{2 n_{c}}\right]^{n_{i} n_{j} \ldots n_{L}-1}-\tilde{\mathcal{I}}\left[q^{2 n_{c}}\right]^{n_{i}-1 n_{j} \ldots n_{L}}\right),
\end{aligned}
$$


where $\Delta_{i j}^{2}=M_{i}^{2}-M_{j}^{2}$. For convenience we have included the reduction algorithm and the basis integrals in the Mathematica file LoopFunctions.m of the arXiv submission with the correspondence

$$
\tilde{\mathcal{I}}\left[q^{2 n_{c}}\right]_{i j \ldots 0}^{n_{i} n_{j} \ldots n_{L}} \equiv J\left[n_{c},\left\{\left\{M_{i}, n_{i}\right\},\left\{M_{j}, n_{j}\right\}, \ldots\right\}, n_{L}\right]
$$

\section{Useful relations for spinors and $\mathrm{SU}(N)$ groups}

We define the charge conjugate $\psi^{C}$ of a 4 -spinor $\psi$ as

$$
\psi^{C} \equiv \mathcal{C} \bar{\psi}^{T}, \quad \overline{\psi^{C}}=\psi^{T} \mathcal{C},
$$

where $\mathcal{C}$ is the charge conjugation operator and $\bar{\psi}=\psi^{\dagger} \gamma^{0}$. It follows from this definition that

$$
\left(\psi_{R}\right)^{C}=\mathcal{C}{\overline{\psi_{L}}}^{T}, \quad\left(\psi_{L}\right)^{C}=\mathcal{C}{\overline{\psi_{R}}}^{T}
$$

The following properties of $\mathcal{C}$ hold in the Dirac and Weyl representation:

$$
\begin{aligned}
\mathcal{C} & =i \gamma^{2} \gamma^{0}, \\
\mathcal{C} & =-\mathcal{C}^{-1}=-\mathcal{C}^{\dagger}=-\mathcal{C}^{T} \\
\mathcal{C} \gamma^{\mu} \mathcal{C}^{-1} & =-\left(\gamma^{\mu}\right)^{T} \\
\mathcal{C} \gamma^{5} \mathcal{C}^{-1} & =\left(\gamma^{5}\right)^{T}=\gamma^{5} \\
\mathcal{C} \gamma^{5} \gamma^{\mu} \mathcal{C}^{-1} & =\left(\gamma^{5} \gamma^{\mu}\right)^{T}=\left(\gamma^{\mu}\right)^{T} \gamma^{5}, \\
\mathcal{C} P_{L} \mathcal{C}^{-1} & =\left(P_{L}\right)^{T}=P_{L}, \\
\mathcal{C} P_{R} \mathcal{C}^{-1} & =\left(P_{R}\right)^{T}=P_{R} .
\end{aligned}
$$

In our formalism we require that if a model contains Dirac spinors $\psi$, then the Lagrangian is expressed in terms of $\psi$ and $\bar{\psi}$. If the model contains Majorana spinors $\lambda$, we require that the Lagrangian is expressed only in terms of $\lambda$, but not in terms of $\bar{\lambda}$. Note that $\bar{\lambda}$ can always be rewritten as

$$
\bar{\lambda}=\left(\lambda^{C}\right)^{T} \mathcal{C}=\lambda^{T} \mathcal{C}
$$

because for Majorana fermions $\lambda^{C}=\lambda$. When contracting spinor indices the following identity may be used

$$
\psi^{T} \Gamma^{T} \bar{\psi}^{T}=-\bar{\psi} \Gamma \psi
$$

A useful relation for the generators $T^{a}$ of the fundamental representation of $\mathrm{SU}(N)$ is

$$
T_{i j}^{a} T_{k l}^{a}=\frac{1}{2}\left(\delta_{i l} \delta_{j k}-\frac{1}{N} \delta_{i j} \delta_{k l}\right)
$$




\section{Dimensional regularization and dimensional reduction}

Throughout this publication we have assumed that the models are regularized in dimensional regularization (DREG) [37], where loop calculations are performed in a quasi- $d$ dimensional space $Q d S$ with the metric tensor $g^{\mu \nu}$ with the property

$$
g^{\mu \nu} g_{\mu \nu}=d=4-\epsilon .
$$

Although DREG is suited for non-supersymmetric models, it is cumbersome to use in supersymmetric models, as it explicitly breaks supersymmetry [44]. For supersymmetric models regularization by dimensional reduction (DRED) [33] is more suited, because it is currently known to not break supersymmetry up to the three-loop level [39, 45, 46]. In DRED the quasi-4-dimensional space, denoted as $Q 4 S$, is decomposed into a quasi- $d$ dimensional space $Q d S$ and a quasi- $\epsilon$-dimensional space $Q \epsilon S$, as $Q 4 S=Q d S \oplus Q \epsilon S$ [39]. The corresponding 4- and $\epsilon$-dimensional metrics are denoted as $\stackrel{\circ}{g}^{\mu \nu}$ and $\breve{g}^{\mu \nu}$, respectively, and the following properties hold:

$$
\begin{aligned}
\stackrel{\circ}{g}^{\mu \nu} & =g^{\mu \nu}+\breve{g}^{\mu \nu}, \\
\breve{g}_{\sigma}^{\mu} \stackrel{\circ}{g}^{\sigma \nu} & =\breve{g}^{\mu \nu}, \\
g_{\sigma}^{\mu} \stackrel{\circ}{g}^{\sigma \nu} & =g^{\mu \nu}, \\
\stackrel{g}{g}^{\mu \nu} \stackrel{\circ}{g}_{\mu \nu} & =4, \\
g^{\mu \nu} g_{\mu \nu} & =d, \\
\breve{g}^{\mu \nu} \breve{g}_{\mu \nu} & =\epsilon, \\
\breve{g}^{\mu \nu} g_{\mu \nu} & =0, \\
\operatorname{tr}\left(\gamma^{\mu} \gamma_{\mu}\right) & =4 d .
\end{aligned}
$$

Open Access. This article is distributed under the terms of the Creative Commons Attribution License (CC-BY 4.0), which permits any use, distribution and reproduction in any medium, provided the original author(s) and source are credited.

\section{References}

[1] ATLAS collaboration, Observation of a new particle in the search for the standard model Higgs boson with the ATLAS detector at the LHC, Phys. Lett. B 716 (2012) 1 [arXiv: 1207.7214] [INSPIRE].

[2] CMS collaboration, Observation of a new boson at a mass of $125 \mathrm{GeV}$ with the CMS experiment at the LHC, Phys. Lett. B 716 (2012) 30 [arXiv:1207.7235] [INSPIRE].

[3] Muon G-2 collaboration, Final report of the muon E821 anomalous magnetic moment measurement at BNL, Phys. Rev. D 73 (2006) 072003 [hep-ex/0602035] [INSPIRE].

[4] F. Jegerlehner, The muon $g-2$ in progress, Acta Phys. Polon. B 49 (2018) 1157 [arXiv: 1804.07409] [INSPIRE].

[5] H.E. Haber and G.L. Kane, The search for supersymmetry: probing physics beyond the standard model, Phys. Rept. 117 (1985) 75 [INSPIRE]. 
[6] B.C. Allanach and A. Voigt, Uncertainties in the lightest CP even Higgs boson mass prediction in the minimal supersymmetric standard model: fixed order versus effective field theory prediction, Eur. Phys. J. C 78 (2018) 573 [arXiv:1804.09410] [INSPIRE].

[7] E. Bagnaschi et al., MSSM Higgs boson searches at the LHC: benchmark scenarios for run 2 and beyond, Eur. Phys. J. C 79 (2019) 617 [arXiv: 1808.07542] [INSPIRE].

[8] A. Drozd, J. Ellis, J. Quevillon and T. You, The universal one-loop effective action, JHEP 03 (2016) 180 [arXiv: 1512.03003] [INSPIRE].

[9] S.A.R. Ellis, J. Quevillon, T. You and Z. Zhang, Extending the universal one-loop effective action: heavy-light coefficients, JHEP 08 (2017) 054 [arXiv: 1706.07765] [INSPIRE].

[10] B. Summ and A. Voigt, Extending the universal one-loop effective action by regularization scheme translating operators, JHEP 08 (2018) 026 [arXiv: 1806.05171] [INSPIRE].

[11] M.K. Gaillard, The Effective One Loop Lagrangian With Derivative Couplings, Nucl. Phys. B 268 (1986) 669 [INSPIRE].

[12] O. Cheyette, Effective action for the standard model with large Higgs mass, Nucl. Phys. B 297 (1988) 183 [INSPIRE].

[13] N. Haba, K. Kaneta, S. Matsumoto and T. Nabeshima, A simple method of calculating effective operators, Acta Phys. Polon. B 43 (2012) 405 [arXiv:1106.6106] [InSPIRE].

[14] B. Henning, X. Lu and H. Murayama, How to use the Standard Model effective field theory, JHEP 01 (2016) 023 [arXiv:1412.1837] [INSPIRE].

[15] B. Henning, X. Lu and H. Murayama, One-loop matching and running with covariant derivative expansion, JHEP 01 (2018) 123 [arXiv:1604.01019] [INSPIRE].

[16] S.A.R. Ellis, J. Quevillon, T. You and Z. Zhang, Mixed heavy-light matching in the universal one-loop effective action, Phys. Lett. B 762 (2016) 166 [arXiv: 1604.02445] [INSPIRE].

[17] J. Fuentes-Martin, J. Portoles and P. Ruiz-Femenia, Integrating out heavy particles with functional methods: a simplified framework, JHEP 09 (2016) 156 [arXiv:1607.02142] [INSPIRE].

[18] Z. Zhang, Covariant diagrams for one-loop matching, JHEP 05 (2017) 152 [arXiv: 1610.00710] [INSPIRE].

[19] F. Staub, From superpotential to model files for FeynArts and CalcHep/CompHEP, Comput. Phys. Commun. 181 (2010) 1077 [arXiv:0909.2863] [INSPIRE].

[20] F. Staub, Automatic calculation of supersymmetric renormalization group equations and self energies, Comput. Phys. Commun. 182 (2011) 808 [arXiv:1002.0840] [INSPIRE].

[21] F. Staub, SARAH 3.2: Dirac gauginos, UFO output and more, Comput. Phys. Commun. 184 (2013) 1792 [arXiv: 1207.0906] [INSPIRE].

[22] F. Staub, SARAH 4: a tool for (not only SUSY) model builders, Comput. Phys. Commun. 185 (2014) 1773 [arXiv: 1309.7223] [INSPIRE].

[23] P. Athron et al., FlexibleSUSY - A spectrum generator generator for supersymmetric models, Comput. Phys. Commun. 190 (2015) 139 [arXiv:1406.2319] [InSPIRE].

[24] P. Athron et al., FlexibleSUSY 2.0: extensions to investigate the phenomenology of SUSY and non-SUSY models, Comput. Phys. Commun. 230 (2018) 145 [arXiv:1710. 03760] [InSPIRE]. 
[25] S. Das Bakshi, J. Chakrabortty and S.K. Patra, CoDEx: Wilson coefficient calculator connecting SMEFT to UV theory, Eur. Phys. J. C 79 (2019) 21 [arXiv:1808.04403] [INSPIRE].

[26] S. Das Bakshi, J. Chakrabortty and S.K. Patra, CoDEx: BSM physics being realised as an SMEFT, in Theory report on the 11th FCC-ee workshop, A Blondel et al. eds., arXiv: 1905.05078.

[27] R.D. Ball, Chiral gauge theory, Phys. Rept. 182 (1989) 1 [inSPIRE].

[28] M. Beneke and V.A. Smirnov, Asymptotic expansion of Feynman integrals near threshold, Nucl. Phys. B 522 (1998) 321 [hep-ph/9711391] [INSPIRE].

[29] B. Jantzen, Foundation and generalization of the expansion by regions, JHEP 12 (2011) 076 [arXiv: 1111.2589] [INSPIRE].

[30] C.G. Callan Jr., Broken scale invariance in scalar field theory, Phys. Rev. D 2 (1970) 1541 [INSPIRE].

[31] K. Symanzik, Small distance behavior in field theory and power counting, Commun. Math. Phys. 18 (1970) 227 [INSPIRE].

[32] E. Bagnaschi, G.F. Giudice, P. Slavich and A. Strumia, Higgs mass and unnatural supersymmetry, JHEP 09 (2014) 092 [arXiv: 1407.4081] [INSPIRE].

[33] W. Siegel, Supersymmetric dimensional regularization via dimensional reduction, Phys. Lett. B 84 (1979) 193.

[34] C.G. Bollini and J.J. Giambiagi, Dimensional renormalization: the number of dimensions as a regularizing parameter, Nuovo Cim. B 12 (1972) 20 [INSPIRE].

[35] J.F. Ashmore, A method of gauge invariant regularization, Lett. Nuovo Cim. 4 (1972) 289 [INSPIRE].

[36] G.M. Cicuta and E. Montaldi, Analytic renormalization via continuous space dimension, Lett. Nuovo Cim. 4 (1972) 329 [INSPIRE].

[37] G. 't Hooft and M.J.G. Veltman, Regularization and renormalization of gauge fields, Nucl. Phys. B 44 (1972) 189 [INSPIRE].

[38] G. 't Hooft, Dimensional regularization and the renormalization group, Nucl. Phys. B 61 (1973) 455 [INSPIRE].

[39] D. Stöckinger, Regularization by dimensional reduction: consistency, quantum action principle and supersymmetry, JHEP 03 (2005) 076 [hep-ph/0503129] [INSPIRE].

[40] E. Bagnaschi, J. Pardo Vega and P. Slavich, Improved determination of the Higgs mass in the MSSM with heavy superpartners, Eur. Phys. J. C 77 (2017) 334 [arXiv:1703.08166] [INSPIRE].

[41] R. Huo, Effective field theory of integrating out sfermions in the MSSM: complete one-loop analysis, Phys. Rev. D 97 (2018) 075013 [arXiv: 1509.05942] [INSPIRE].

[42] J. Aebischer, A. Crivellin, C. Greub and Y. Yamada, The MSSM without gluinos; an effective field theory for the stop sector, Eur. Phys. J. C 77 (2017) 740 [arXiv:1703.08061] [INSPIRE].

[43] I. Jack et al., Decoupling of the epsilon scalar mass in softly broken supersymmetry, Phys. Rev. D 50 (1994) R5481 [hep-ph/9407291] [INSPIRE]. 
[44] R. Delbourgo and V.B. Prasad, Supersymmetry in the four-dimensional limit, J. Phys. G 1 (1975) 377 [INSPIRE].

[45] D.M. Capper, D.R.T. Jones and P. van Nieuwenhuizen, Regularization by dimensional reduction of supersymmetric and nonsupersymmetric gauge theories, Nucl. Phys. B 167 (1980) 479 [INSPIRE].

[46] D. Stöckinger and J. Unger, Three-loop MSSM Higgs-boson mass predictions and regularization by dimensional reduction, Nucl. Phys. B 935 (2018) 1 [arXiv:1804.05619] [INSPIRE]. 